TRANSACTIONS OF THE

AMERICAN MATHEMATICAL SOCIETY

Volume 358, Number 10, October 2006, Pages 4279-4341

S 0002-9947(05)03773-6

Article electronically published on August 1, 2005

\title{
ROOT INVARIANTS IN THE ADAMS SPECTRAL SEQUENCE
}

\author{
MARK BEHRENS
}

\begin{abstract}
Let $E$ be a ring spectrum for which the $E$-Adams spectral sequence converges. We define a variant of Mahowald's root invariant called the 'filtered root invariant' which takes values in the $E_{1}$ term of the E-Adams spectral sequence. The main theorems of this paper are concerned with when these filtered root invariants detect the actual root invariant, and explain a relationship between filtered root invariants and differentials and compositions in the $E$-Adams spectral sequence. These theorems are compared to some known computations of root invariants at the prime 2 . We use the filtered root invariants to compute some low-dimensional root invariants of $v_{1}$-periodic elements at the prime 3 . We also compute the root invariants of some infinite $v_{1}$-periodic families of elements at the prime 3 .
\end{abstract}

\section{INTRODUCTION}

In his work on metastable homotopy groups [22, Mahowald introduced an invariant that associates to every element $\alpha$ in the stable stems a new element $R(\alpha)$ called the root invariant of $\alpha$. The construction has indeterminacy and so $R(\alpha)$ is in general only a coset. The main result of [25] indicates a deep relationship between elements of the stable homotopy groups of spheres which are root invariants and their behavior in the EHP spectral sequence. Mahowald and Ravenel conjecture in 24] that, loosely speaking, the root invariant of a $v_{n}$-periodic element is $v_{n+1}$-periodic. Thus the root invariant is related simultaneously to unstable and chromatic phenomena.

The conjectural relationship between root invariants and the chromatic filtration is based partly on computational evidence. For instance, at the prime 2, we have [25, 18

$$
R\left(2^{i}\right)= \begin{cases}\alpha_{4 t}, & i=4 t, \\ \alpha_{4 t+1}, & i=4 t+1, \\ \alpha_{4 t+1} \alpha_{1}, & i=4 t+2, \\ \alpha_{4 t+1} \alpha_{1}^{2}, & i=4 t+3,\end{cases}
$$

while at odd primes we have [35, 25]

$$
R\left(p^{i}\right)=\alpha_{i},
$$

demonstrating that the root invariant sends $v_{0}$-periodic families to $v_{1}$-periodic families. For $p \geq 5$ it is also known [35, 25] that

$$
\beta_{i} \in R\left(\alpha_{i}\right)
$$

Received by the editors November 4, 2003 and, in revised form, June 16, 2004.

2000 Mathematics Subject Classification. Primary 55Q45; Secondary 55Q51, 55T15.

The author was partially supported by the NSF. 
and

$$
\beta_{p / 2} \in R\left(\alpha_{p / 2}\right) .
$$

These computations led the authors of 25 to regard the root invariants as defining the $n^{\text {th }}$ Greek letter elements as the root invariants of the $(n-1)^{\text {st }}$ Greek letter elements when the relevant Smith-Toda complexes do not exist.

Other evidence of the root invariant raising chromatic filtration is seen in the cohomology of the Steenrod algebra. Mahowald and Shick define a chromatic filtration on $\operatorname{Ext}_{A}\left(\mathbb{F}_{2}, \mathbb{F}_{2}\right)$ in [26], and Shick proves that an algebraic version of the root invariant increases chromatic filtration in this context in [36].

The Tate spectrum computations of [11, [10], 27, [16], 2], and 15] indicate that the $\mathbb{Z} / p$-Tate spectrum of a $v_{n}$-periodic cohomology theory is $v_{n}$-torsion. The root invariant is defined using the Tate spectrum of the sphere spectrum, so the results of the papers listed above provide even more evidence that the root invariant of a $v_{n}$-periodic element should be $v_{n}$-torsion.

The purpose of this paper is to introduce a new variant of the root invariant called the filtered root invariant. We apply the filtered root invariant towards the computation of some new root invariants at the prime 3 in low dimensions. We also compute the root invariants of some infinite $v_{1}$-families as infinite $v_{2}$-families at the prime 3. We will also describe how the theory of filtered root invariants given in this paper works out to give an alternative perspective on known computations of root invariants at the prime 2 .

Let $X$ be a finite $p$-local spectrum, and let $E$ be a ring spectrum for which the $E$-Adams spectral sequence (E-ASS) converges. Given $\alpha \in \pi_{t}(X)$, we define its filtered root invariants $R_{E}^{[k]}(\alpha)$ to be a sequence of cosets of $d_{r}$-cycles in the $E_{1}^{k, *}$ term of the $E$-ASS converging to $\pi_{*}(X)$, where $r$ depends on $k$. These invariants govern the passage between the $E$-root invariant $R_{E}(\alpha)$, or the algebraic root invariant $R_{a l g}(\alpha)$, and the elements in the $E$-ASS that detect $R(\alpha)$. The passage of each of these filtered root invariants to the next is governed by differentials and compositions in the E-ASS. Our method is to use algebraic and E-cohomology computations to determine the first of these filtered root invariants and then iteratively deduce the higher ones from differentials and compositions. Under favorable circumstances, the last of these filtered root invariants will detect the root invariant.

In Section 2, we discuss the E-Adams resolution, and various filtered forms of the Tate spectrum which we shall be using to define the the filtered root invariants.

In Section 3, we define the filtered root invariants. We also recall the definitions of the root invariant, the $E$-root invariant, and the algebraic root invariant. We will sometimes refer to the root invariant as the "homotopy root invariant" to distinguish it from all of the other variants being used.

Our main results are most conveniently stated using the language of Toda brackets introduced in the appendix of [37. In Section 4 , we define a variant of the Toda bracket, the $K$-Toda bracket, which is taken to be an attaching map in a fixed finite $\mathrm{CW}$-complex $K$. Some properties of $K$-Toda brackets are introduced. We also define a version on the $E_{r}$ term of the $E$-ASS.

In Section 5 , we state the main results which relate the filtered root invariants to root invariants, Adams differentials, compositions, $E$-root invariants, and algebraic root invariants. If $R_{E}^{[k]}(\alpha)$ contains a permanent cycle $x$, then $x$ detects an element of $R(\alpha)$ modulo Adams filtration $k+1$. If $R_{E}^{[k]}(\alpha)$ does not contain a permanent cycle, there is a formula relating its $E$-Adams differential to the next filtered root 
invariant. Thus if one knows a filtered root invariant, one may sometimes deduce the next one from its $E$-Adams differential. There is a similar result concerning compositions. The zeroth filtered root invariant $R_{E}^{[0]}(\alpha)$ is the $E$-root invariant. The first filtered root invariant $R_{E}^{[1]}(\alpha)$ is the $E \wedge \bar{E}$-root invariant $(\bar{E}$ is the fiber of the unit of $E$ ). If $E=H \mathbb{F}_{p}$, then the first non-trivial filtered root invariant is the algebraic root invariant $R_{\text {alg }}(\alpha)$.

We present the proofs of the main theorems of Section 5 in Section 6 The proofs are technical, and it is for this reason that they have been relegated to their own section.

In Section 7 we give examples of these theorems with $E=b o$, and show how the bo resolution computes the root invariants of the elements $2^{k}$ at the prime 2 . This was the motivating example for this project.

We will need to make some extensive computations in an algebraic AtiyahHirzebruch spectral sequence (AAHSS). This is the spectral sequence obtained by applying $\operatorname{Ext}_{B P_{*} B P}\left(B P_{*}, B P_{*}(-)\right)$ to the cellular filtration of projective space. We introduce this spectral sequence in Section 8 We compute the $d_{r}$ differentials for small $r$ using formal group methods.

Calculating homotopy root invariants from our filtered root invariants is a delicate business. In an effort to make our low-dimensional calculations easier to follow and less ad hoc, we spell out our methodology in the form of Procedure 9.1, which we follow throughout our low-dimensional calculations. The description of this procedure is the subject of Section 9 .

In Section 10, we compute some $B P$-filtered root invariants of the elements $p^{i}$ as well as of the elements $\alpha_{i / j}$. We find that at every odd prime $p$

$$
\begin{gathered}
\pm \alpha_{i} \in R_{B P}^{[1]}\left(p^{i}\right), \\
\pm \beta_{i / j} \in R_{B P}^{[2]}\left(\alpha_{i / j}\right) .
\end{gathered}
$$

These filtered root invariants hold at the prime 2 modulo an indeterminacy which is identified, but not computed, in this paper. The only exceptions are the cases $i=j=1$ and $i=j=2$ at the prime 2 (these cases correspond to the existence of the Hopf invariant 1 elements $\nu$ and $\sigma$ ). These filtered root invariants are computed by means of manipulation of formulas in $B P_{*} B P$ arising from $p$-typical formal groups.

In Section 11 we compute the root invariants

$$
R\left(\beta_{1}\right)=\beta_{1}^{p}
$$

at primes $p>2$. These root invariants were announced without proof in [25]. This computation is accomplished by applying our theorems to the Toda differential in the Adams-Novikov spectral sequence (ANSS).

In Section 12, we apply the methods described to compute some root invariants of the Greek letter elements $\alpha_{i / j}$ that lie within the 100-stem at the prime 3. At the prime $3, \beta_{i}$ is known to be a permanent cycle for $i \equiv 0,1,2,5,6(\bmod 9)$ [] and is conjectured to exist for $i \equiv 3(\bmod 9)$. The element $\beta_{3}$ is a permanent cycle. One might expect from the previous work for $p \geq 5$ that $\beta_{i} \in R\left(\alpha_{i}\right)$ when $\beta_{i}$ exists. Surprisingly, there is at least one instance where $\beta_{i}$ exists, yet is not contained in $R\left(\alpha_{i}\right)$. Our low-dimensional computations of $R\left(\alpha_{i / j}\right)$ at $p=3$ are summarized in Table 1 .

All of these root invariants are $v_{2}$-periodic in the sense that they are detected in $\pi_{*}\left(L_{2} S^{0}\right)$ [34. A similar phenomenon happens at the prime 2 with the root 
TABLE 1. Low-dimensional root invariants of $\alpha_{i / j}$ at $p=3$

\begin{tabular}{c|c}
\hline Element & Root Invariant \\
\hline$\alpha_{1}$ & $\beta_{1}$ \\
$\alpha_{2}$ & $\pm \beta_{1}^{2} \alpha_{1}$ \\
$\alpha_{3 / 2}$ & $-\beta_{3 / 2}$ \\
$\alpha_{3}$ & $\beta_{3}$ \\
$\alpha_{4}$ & $\pm \beta_{1}^{5}$ \\
$\alpha_{5}$ & $\beta_{5}$ \\
$\alpha_{6 / 2}$ & $\beta_{6 / 2}$ \\
$\alpha_{6}$ & $-\beta_{6}$ \\
\hline
\end{tabular}

invariants of $2^{i}$ : they are not all given by the Greek letter elements $\alpha_{i}$, but the elements $R\left(2^{i}\right)$ are nevertheless $v_{1}$-periodic [25]. The lesson we learn is that if one believes that the homotopy Greek letter elements should be determined by iterated root invariants (as suggested in [25]), then they will not always agree with the algebraic Greek letter elements, even when the latter are permanent cycles.

These results will be partly generalized to compute $R\left(\alpha_{i}\right)$ for $i \equiv 0,1,5(\bmod 9)$ at the prime 3 in Section 15. The remainder of this paper is devoted to providing the machinery necessary for this computation.

In the ANSS the $\beta$ family lies in low Adams-Novikov filtration, but in the ASS this family is in high filtration. For the purposes of infinite chromatic families, it is often useful to take both spectral sequences into account simultaneously. In Section 13 we explain how our framework can be applied to the Mahowald spectral sequence to compute algebraic root invariants. As mentioned earlier, algebraic root invariants are the first non-trivial $H \mathbb{F}_{p}$ filtered root invariants.

Infinite families of Greek letter elements are constructed as homotopy classes through the use of Smith-Toda complexes. In their computation of $R\left(\alpha_{i}\right)$ for $p \geq 5$, Mahowald and Ravenel introduce modified root invariants [25] which take values in the homotopy groups of certain Smith-Toda complexes. In Section 14 we adapt our results to modified root invariants.

Our modified root invariant methods are applied in Section 15 to make some new computations of the root invariants of some infinite $v_{1}$-periodic families at the prime 3. Specifically, we are able to show that

$$
(-1)^{i+1} \beta_{i} \in R\left(\alpha_{i}\right)
$$

for $i \equiv 0,1,5(\bmod 9)$.

This paper represents the author's dissertation work. The author extends his heartfelt gratitude to his adviser, J. Peter May, for his guidance and encouragement, and to Mark Mahowald, for many enlightening conversations regarding the contents of this paper.

Conventions. Throughout this paper we will be working in the stable homotopy category localized at some prime $p$. We will always denote the quantity $q=2(p-1)$, as usual. All ordinary homology will be taken with $\mathbb{F}_{p}$ coefficients. If $p=2$, let $P_{N}^{M}$ denote the stunted projective space with bottom cell in dimension $N$ and top cell in dimension $M$. Here $M$ and $N$ may be infinite or negative. See [25] for details. If $p$ is odd, then projective space is replaced by $B \Sigma_{p}$. The complex $B \Sigma_{p}$ has a stable cell in every positive dimension congruent to 0 or $-1 \bmod q$, and we will use the 
notation $P_{N}^{M}$ to indicate the stunted complex with cells in dimensions between $N$ and $M$. When $M=\infty$ or when $N=-\infty$, the superscript or subscript may be omitted.

Given a spectrum $E$, the Tate spectrum

$$
\Sigma(E \wedge P)_{-\infty}=\Sigma \operatorname{holim}\left(E \wedge P_{-n}\right)
$$

will be denoted $t E$. To relate this notation to that in [15], we have

$$
t E=t\left(\iota_{*} E\right)^{\mathbb{Z} / p} .
$$

There is a unit $S^{0} \rightarrow t E$. For $E=S^{0}$, this is the inclusion of the 0 -cell. If $X$ is a finite complex, then the Segal conjecture for the group $\mathbb{Z} / p[3$, ,9], 32] (also known as Lin's Theorem [20, 21] at $p=2$ and Gunawardena's Theorem [17] for $p>2$ ) implies that the map

$$
X=X \wedge S^{0} \rightarrow X \wedge t S^{0}=t X
$$

is $p$-completion (the last equality requires $X$ to be finite).

Suppose $A$ and $B$ are two subsets of a set $C$. We shall write $A \cong B$ to indicate that $A \cap B$ is nonempty. This is useful notation when dealing with operations with indeterminacy. If we are working over a ring $R$, we shall use the notation $\doteq$ to indicate that two quantities are equal modulo multiplication by a unit in $R^{\times}$. We shall similarly use the notation $\dot{\epsilon}$ for containment up to multiplication by a unit.

We will denote the regular ideal $\left(p, v_{1}, v_{2}, \ldots, v_{n-1}\right) \subseteq B P_{*}$ by $I_{n}$.

Finally, we will be using the following abbreviations for spectral sequences:

ASS: The classical Adams spectral sequence.

ANSS: The Adams-Novikov spectral sequence derived from $B P$.

$E$-ASS: The generalized Adams spectral sequence derived from a ring spectrum $E$.

AHSS: The Atiyah-Hirzebruch spectral sequence. We will be using the form that computes stable homotopy groups from homology.

AAHSS: The algebraic Atiyah-Hirzebruch spectral sequence, which uses the cellular filtration to compute $\operatorname{Ext}(X)$.

MSS: The Mahowald spectral sequence, which computes $\operatorname{Ext}(X)$ by applying Ext(-) to an Adams resolution of $X$.

\section{Filtered Tate spectra}

Given a ring spectrum $E$, we will establish some notation for dealing with the $E$ Adams resolution. We will then mix the Adams filtration with the skeletal filtration in the Tate spectrum $t S^{0}$. These filtered Tate spectra will carry the filtered root invariants defined in Section 3, Our treatment of the Adams resolution closely follows that of Bruner in [8, IV.3].

For $E$ a ring spectrum, let $\bar{E}$ be the fiber of the unit, so there is a cofiber sequence

$$
\bar{E} \rightarrow S^{0} \stackrel{\eta}{\rightarrow} E .
$$

For $X$ a spectrum, let $W_{k}(X)$ denote the $k$-fold smash power $\bar{E}^{(k)} \wedge X$. We shall also use the notation $W_{k}^{l}(X)$ to denote the cofiber

$$
W_{k+l+1}(X) \rightarrow W_{k}(X) \rightarrow W_{k}^{l}(X) .
$$


We may drop the $X$ from the notation when $X=S^{0}$. Note that with our definitions $W_{k}^{k-1}(X) \simeq *$. The $E$-Adams resolution of $X$ now takes the form

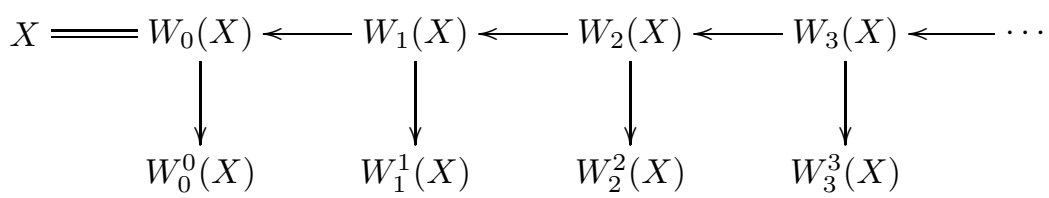

The notation $W_{k}^{l}(X)$ is used because the E-ASS for $W_{k}^{l}(X)$ is obtained from the $E$-ASS for $X$ by setting $E_{1}^{s, t}=0$ for $s<k$ and $s>l$, and adjusting the differentials accordingly. If the resolution converges to the $p$-completion ( $p$-localization) of $X$, then $W_{\infty}(X) \simeq *$ in the $p$-complete ( $p$-local) stable homotopy category, and $W_{s}^{\infty}(X) \simeq W_{s}(X)$.

We shall denote $E_{r}(X)$ for the $E_{r}$ term of the $E$-ASS for $X$. An element of $E_{1}^{s, t}(X)=\pi_{t-s}\left(W_{s}^{s}(X)\right)$ is a $d_{r}$-cycle if and only if it lifts to an element of $\pi_{t-s}\left(W_{s}^{s+r}\right)$. Given an element $\alpha \in \pi_{n}(X)$ we shall let $f_{i l t}(\alpha)$ denote its $E$ Adams filtration.

In what follows, the reader may find it helpful to assume that the spectra $W_{s}$ are CW spectra and the maps

$$
W_{s} \rightarrow W_{s-1}
$$

are the inclusions of subcomplexes. If this is the case, then in what follows the homotopy colimits may simply be regarded as unions. As Bruner points out 8 , IV.3.1], this assumption represents no loss of generality, since any infinite tower may be replaced with a tower of inclusions of CW-spectra through the use of CW approximation and mapping telescopes. The Tate spectrum $\Sigma^{-1} t S^{0}$ is bifiltered, as depicted in the following diagram:

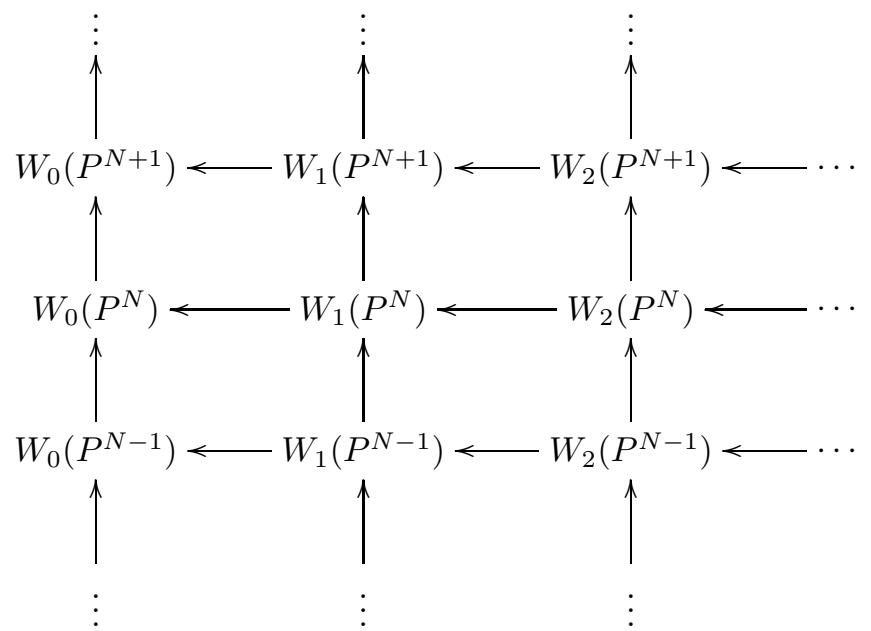

In the above diagram, $W_{k}\left(P^{N}\right)$ is the spectrum $W_{k}\left(P^{N}\right)_{-\infty}=\operatorname{holim}_{M} W_{k}\left(P_{-M}^{N}\right)$, where the homotopy inverse limit is taken after smashing with $W_{k}$. We emphasize that this is in general quite different from what one obtains if one smashes with $W_{k}$ after taking the homotopy inverse limit. 
Given increasing sequences of integers

$$
\begin{aligned}
& I=\left\{k_{1}<k_{2}<\cdots<k_{l}\right\}, \\
& J=\left\{N_{1}<N_{2}<\cdots<N_{l}\right\}
\end{aligned}
$$

we define subsets $S(I, J)$ of $\mathbb{Z} \times \mathbb{Z}$ by

$$
S(I, J)=\bigcup_{i=1}^{l}\left\{(a, b): a \geq k_{i}, b \leq N_{i}\right\} .
$$

We give the set of all multi-indices $(I, J)$ the structure of a poset by declaring $(I, J) \leq\left(I^{\prime}, J^{\prime}\right)$ if and only if $S(I, J) \subseteq S\left(I^{\prime}, J^{\prime}\right)$.

Definition 2.1 (Filtered Tate spectrum). Given sequences

$$
\begin{aligned}
I & =\left\{k_{1}<k_{2}<\cdots<k_{l}\right\}, \\
J & =\left\{N_{1}<N_{2}<\cdots<N_{l}\right\}
\end{aligned}
$$

with $k_{i} \geq 0$, we define the filtered Tate spectrum (of the sphere) as the homotopy colimit

$$
W_{I}\left(P^{J}\right)=\bigsqcup_{i} W_{k_{i}}\left(P^{N_{i}}\right)
$$

We allow for the possibility of $N_{l}=\infty$. More generally, given another pair of sequences $\left(I^{\prime}, J^{\prime}\right) \leq(I, J)$, we define spectra

$$
W_{I}^{I^{\prime}}\left(P_{J^{\prime}}^{J}\right)=\operatorname{cofiber}\left(W_{I^{\prime}+1}\left(P^{J^{\prime}-1}\right) \rightarrow W_{I}\left(P^{J}\right)\right),
$$

where $I^{\prime}+1$ (respectively $J^{\prime}-1$ ) is the sequence obtained by increasing (decreasing) every element of the sequence by 1 .

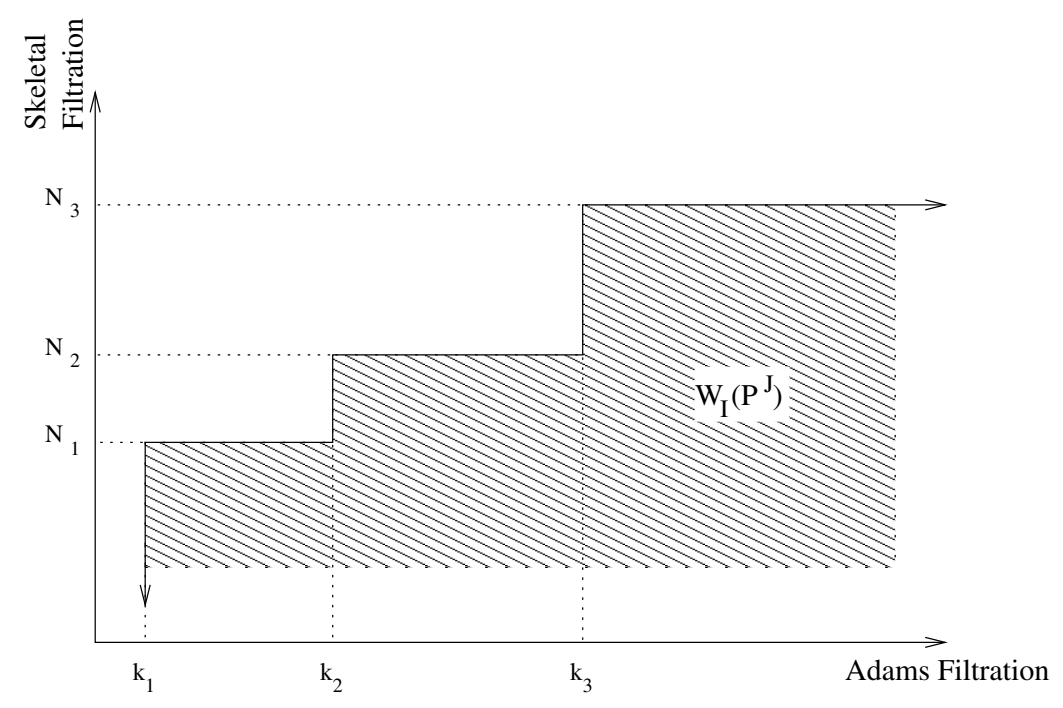

FigurE 1. The filtered Tate spectrum 
Figure 1 displays a diagram of $S(I, J)$ intended to help the reader visualize the filtered Tate spectrum. The entire Tate spectrum is represented by the right halfplane. The shaded region $S(I, J)$ is the portion represented by the filtered Tate spectrum $W_{I}\left(P^{J}\right)$.

\section{Definitions of VARIOUS Forms of THE ROOT InVARIANT}

In this section we will recall the definitions of the Mahowald root invariant, the $E$-root invariant, and the algebraic root invariant. We will then define the filtered root invariants.

Definition 3.1 (Root invariant). Let $X$ be a finite complex, and $\alpha \in \pi_{t}(X)$. The root invariant (also called the Mahowald invariant) of $\alpha$ is the coset of all dotted arrows making the following diagram commute:

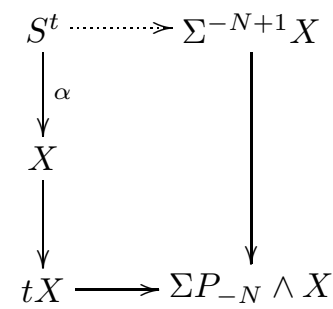

This coset is denoted $R(\alpha)$. Here the map $X \rightarrow t X$ is $p$-completion by the Segal conjecture (1.1), and $N$ is chosen to be minimal such that the composite $S^{t} \rightarrow$ $\Sigma P_{-N} \wedge X$ is non-trivial.

The root invariant is difficult to compute because it involves knowing the homotopy groups of the finite complex $X$ and of $X \wedge P_{-N}$. For this reason, Mahowald and Ravenel [25] introduced a calculable approximation to the root invariant called the $E$-root invariant, for $E$ a ring spectrum. We find it useful to generalize to arbitrary spectra and to elements of $\pi_{*}(E)$.

Definition 3.2 (E-root invariant). Let $E$ be a spectrum. Let $x \in \pi_{t}(E)$. Define the E-root invariant of $x$ to be the coset $R_{E}(\alpha)$ of dotted arrows making the following diagram commute:

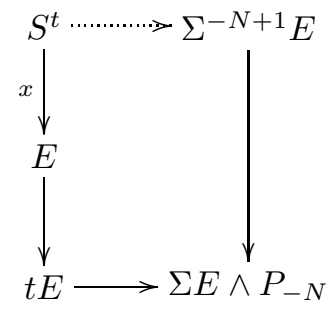

It is quite possible that the composite $S^{t} \rightarrow t E$ is trivial. If this is the case the $E$-root invariant is said to be trivial. Otherwise, in the diagram above we choose $N$ to be minimal such that the composite $S^{t} \rightarrow \Sigma E \wedge P_{-N}$ is non-trivial.

If $E$ is a ring spectrum, and $\alpha \in \pi_{*}(X)$ for $X$ a finite spectrum, let $x=h(\alpha) \in$ $E_{*}(X)$ be the Hurewicz image of $\alpha$. We will then refer to the $E \wedge X$-root invariant of $h(\alpha)$ simply as the $E$-root invariant of $\alpha$. Therefore, by abuse of notation, we have

$$
R_{E}(\alpha)=R_{E \wedge X}(h(\alpha)) .
$$


Finally, one may define root invariants in Ext. These are called algebraic root invariants.

Definition 3.3 (Algebraic root invariant). Let $\alpha$ be an element of $\operatorname{Ext}^{s, t}\left(H_{*} X\right)$. We have the following diagram of Ext groups which defines the algebraic root invariant $R_{\text {alg }}(\alpha)$ :

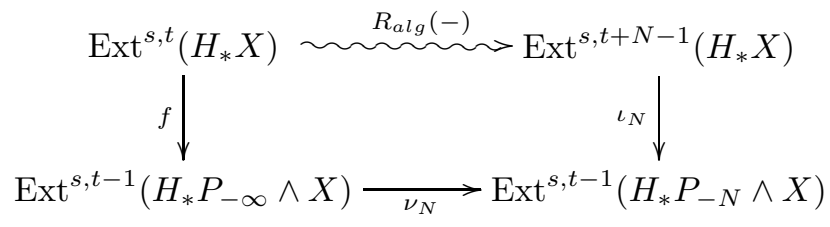

Here $f$ is induced by the inclusion of the -1 -cell of $P_{-\infty}, \nu_{N}$ is the projection onto the $-N$-coskeleton, $\iota_{N}$ is inclusion of the $-N$-cell, and $N$ is minimal with respect to the property that $\nu_{N} \circ f(\alpha)$ is non-zero. Then the algebraic root invariant $R_{\text {alg }}(\alpha)$ is defined to be the coset of lifts $\gamma \in$ Ext $^{s, t+N-1}\left(H_{*} X\right)$ of the element $\nu_{N} \circ f(\alpha)$.

We wish to extend these definitions to a sequence of filtered root invariants that appear in the $E$-Adams resolution. Suppose that $X$ is a finite complex and $\alpha \in \pi_{t}(X)$. We want to lift $\alpha$ over the smallest possible filtered Tate spectrum (Definition 2.1). To this end, we shall describe a pair of sequences

$$
\begin{aligned}
& I=\left\{k_{1}<k_{2}<\cdots<k_{l}\right\}, \\
& J=\left\{-N_{1}<-N_{2}<\cdots<-N_{l}\right\}
\end{aligned}
$$

associated to $\alpha$, which we define inductively. Let $k_{1} \geq 0$ be maximal such that the composite

$$
S^{t-1} \stackrel{\alpha}{\rightarrow} \Sigma^{-1} X \rightarrow \Sigma^{-1} t X \rightarrow W_{0}^{k_{1}-1}(P \wedge X)_{-\infty}
$$

is trivial. Next, choose $N_{1}$ to be maximal such that the composite

$$
S^{t-1} \stackrel{\alpha}{\rightarrow} \Sigma^{-1} X \rightarrow \Sigma^{-1} t X \rightarrow W_{0}^{\left(k_{1}-1, k_{1}\right)}\left(P_{\left(-N_{1}+1, \infty\right)} \wedge X\right)
$$

is trivial. Inductively, given

$$
\begin{aligned}
I^{\prime} & =\left(k_{1}, k_{2}, \ldots, k_{i}\right), \\
J^{\prime} & =\left(-N_{1},-N_{2}, \ldots,-N_{i}\right)
\end{aligned}
$$

let $k_{i+1}$ be maximal so that the composite

$$
S^{t-1} \stackrel{\alpha}{\rightarrow} \Sigma^{-1} X \rightarrow \Sigma^{-1} t X \rightarrow W_{0}^{\left(I^{\prime}-1, k_{i+1}-1\right)}\left(P_{\left(J^{\prime}+1, \infty\right)} \wedge X\right)
$$

is trivial. If there is no such maximal $k_{i+1}$, we declare that $k_{i+1}=\infty$ and we are finished. Otherwise, choose $N_{i+1}$ to be maximal such that the composite

$$
S^{t-1} \stackrel{\alpha}{\rightarrow} \Sigma^{-1} X \rightarrow \Sigma^{-1} t X \rightarrow W_{0}^{\left(I^{\prime}-1, k_{i+1}-1, k_{i+1}\right)}\left(P_{\left(J^{\prime}+1,-N_{i+1}+1, \infty\right)} \wedge X\right)
$$

is trivial, and continue the inductive procedure. We shall refer to the pair $(I, J)$ as the $E$-bifiltration of $\alpha$.

Observe that there is an exact sequence

$$
\pi_{t-1}\left(W_{I}\left(P^{J} \wedge X\right)\right) \rightarrow \pi_{t}(t X) \rightarrow \pi_{t-1}\left(W^{I-1}\left(P_{J+1} \wedge X\right)\right) .
$$

Our choice of $(I, J)$ ensures that the image of $\alpha$ in $\pi_{t-1}\left(W^{I-1}\left(P_{J+1} \wedge X\right)\right)$ is trivial. Thus $\alpha$ lifts to an element $f^{\alpha} \in \pi_{t-1}\left(W_{I}\left(P^{J} \wedge X\right)\right)$. 


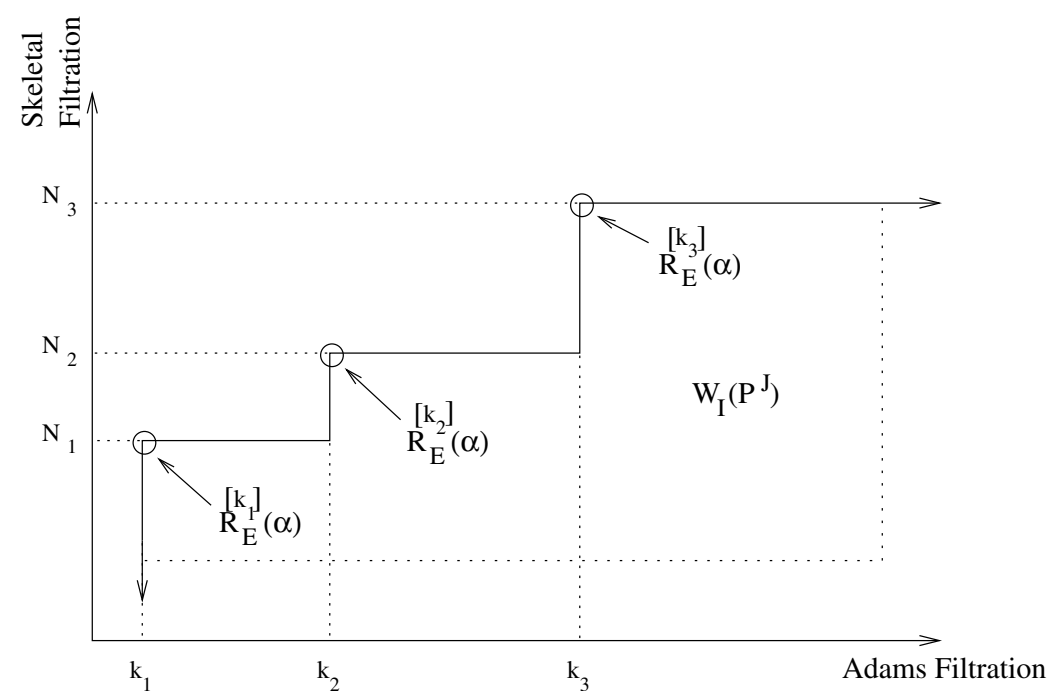

FiguRE 2. The the bifiltration of the filtered root invariants in the filtered Tate spectrum

Definition 3.4 (Filtered root invariants). Let $X$ be a finite complex, let $E$ be a ring spectrum such that the $E$-Adams resolution converges, and let $\alpha$ be an element of $\pi_{t}(X)$ of $E$-bifiltration $(I, J)$. Given a lift $f^{\alpha} \in \pi_{t-1}\left(W_{I}\left(P^{J} \wedge X\right)\right)$, the $k^{\text {th }}$ filtered root invariant is said to be trivial if $k \neq k_{i}$ for any $k_{i} \in I$. Otherwise, if $k=k_{i}$ for some $i$, we say that the image $\beta$ of $f^{\alpha}$ under the collapse map

$$
\pi_{t-1}\left(W_{I}\left(P^{J} \wedge X\right)\right) \rightarrow \pi_{t-1}\left(W_{k_{i}}^{k_{i}}\left(\Sigma^{-N_{i}} X\right)\right)
$$

is an element of the $k^{\text {th }}$ filtered root invariant of $\alpha$. The $k^{\text {th }}$ filtered root invariant is the coset $R_{E}^{[k]}(\alpha)$ of $E_{1}^{k, t+k+N_{i}-1}(X)$ of all such $\beta$ as we vary the lift $f^{\alpha}$.

Remark 3.5. Let $r_{i}$ denote the difference $k_{i+1}-k_{i}$. Then there is a factorization

$$
\pi_{t-1}\left(W_{I}\left(P^{J} \wedge X\right)\right) \rightarrow \pi_{t-1}\left(W_{k_{i}}^{k_{i+1}-1}\left(\Sigma^{-N_{k_{i}}} X\right)\right) \rightarrow \pi_{t-1}\left(W_{k_{i}}^{k_{i}}\left(\Sigma^{-N_{k_{i}}} X\right)\right) .
$$

Thus any such $\beta \in R_{E}^{[k]}(\alpha)$ is actually a $d_{r}$-cycle for $r<r_{i}$.

Figure 2 gives a companion visualization to Figure 1, by displaying the bifiltrations of the filtered root invariants in the filtered Tate spectrum.

\section{The Toda Bracket associated to A COMPlex}

In this section we introduce a variant of the Toda bracket. This treatment is essentially a specialization of the treatment of Toda brackets given in the appendix of [37. Suppose $K$ is a finite $\mathrm{CW}$-spectrum with one bottom-dimensional cell and one top-dimensional cell. Suspend $K$ accordingly so that it is connective and $n$ dimensional with one cell in dimension zero and one cell in dimension $n$. For some other spectrum $X$ we shall define the $K$-Toda bracket to be an operator which, when defined, takes an element of $\pi_{t}(X)$ to a coset of $\pi_{t+n-1}(X)$. We shall present a dual definition, and show this dual definition is equivalent to our original definition. We will also define a variant on the $E_{r}$ term of the $E$-ASS. 
In what follows, we let $K^{j}$ be the $j$-skeleton of $K$, and let $K_{i}^{j}$ be the quotient $K^{j} / K^{i-1}$. We shall omit the top index for the $i$-coskeleton $K_{i}=K / K^{i-1}$.

Definition 4.1 ( $K$-Toda bracket). Let

$$
f: \Sigma^{-1} K_{1} \rightarrow S^{0}
$$

be the attaching map of the 1-coskeleton of $K$ to the 0-cell, so that the cofiber of $f$ is $K$. Let $\nu: K_{1} \rightarrow S^{n}$ be the projection onto the top cell. Suppose $\alpha$ is an element of $\pi_{t}(X)$. We have

$$
\pi_{t}(X) \stackrel{\nu_{*}}{\stackrel{f}{\leftrightarrows}} \pi_{t+n}\left(X \wedge K_{1}\right) \stackrel{f_{*}}{\longrightarrow} \pi_{t+n-1}(X) .
$$

We say the $K$-Toda bracket

$$
\langle K\rangle(\alpha) \subseteq \pi_{t+n-1}(X)
$$

is defined if $\alpha$ is in the image of $\nu_{*}$. Then the $K$-Toda bracket is the collection of all $f_{*}(\gamma) \in \pi_{t+n-1}(X)$, where $\gamma \in \pi_{t+n}\left(X \wedge K_{1}\right)$ is any element satisfying $\nu_{*}(\gamma)=\alpha$. If $\langle K\rangle(\alpha)$ contains only one element, we will say that it is strictly defined.

Remark 4.2. If the $(n-1)$-skeleton $K^{n-1}$ is coreducible, then the attaching map $f$ factors as a composite

$$
f: \Sigma^{-1} K_{1} \stackrel{\nu}{\rightarrow} S^{n-1} \stackrel{\xi}{\rightarrow} S^{0} .
$$

Then the product $\xi \cdot \alpha$ is in $\langle K\rangle(\alpha)$.

We remark on the relationship to Shipley's treatment of Toda brackets in triangulated categories given in the appendix of 37. In Shipley's terminology, if $K$ is an $m$-filtered object in $\left\{f_{1}, f_{2}, \ldots, f_{m-1}\right\}$ with $F_{1} K \simeq S^{0}$ and $F_{m} K / F_{m-1} K \simeq S^{n}$, then we have the equality (modulo indeterminacy)

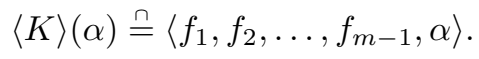

In particular, if we take $K$ to be the 3 -filtered object

$$
* \subseteq S^{0} \subseteq K^{n-1} \subseteq K
$$

with attaching maps

$$
\begin{aligned}
& f_{1}: \Sigma^{-1} K_{1}^{n-1} \rightarrow S^{0}, \\
& f_{2}: S^{n-1} \rightarrow K_{1}^{n-1},
\end{aligned}
$$

then we have the equality (modulo indeterminacy)

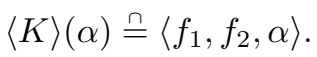

The reason we do not have exact equalities is that with the Toda bracket, you can get more indeterminacy by varying the $m$-filtered object, whereas the $m$-filtered object is fixed in the case of the $K$-Toda bracket. However, the $K$-Toda bracket can also get indeterminacy that is not seen by the Toda bracket if $X \neq S^{0}$, because there are potentially more choices of lift $\gamma$ if you smash with $X$.

In some of our applications the following dual variant will be more natural to work with. Our use of the word 'dual' stems from the use the skeletal instead of coskeletal filtration of $K$. 
Definition 4.3 (Dual definition of the K-Toda bracket). Let

$$
g: S^{n-1} \rightarrow K^{n-1}
$$

be the attaching map of the $n$-cell of $K$ to the $(n-1)$-skeleton, so that the cofiber of $g$ is $K$. Let $\iota: S^{0} \rightarrow K^{n-1}$ be the inclusion of the bottom cell. Suppose $\alpha$ is an element of $\pi_{t}(X)$. We have

$$
\pi_{t}(X) \stackrel{g_{*}}{\longrightarrow} \pi_{t+n-1}\left(X \wedge K^{n-1}\right) \stackrel{\iota_{*}}{\longleftarrow} \pi_{t+n-1}(X) .
$$

We say the (dual) $K$-Toda bracket

$$
\langle K\rangle(\alpha) \subseteq \pi_{t+n-1}(X)
$$

is defined if $g_{*}(\alpha)$ is in the image of $\iota_{*}$. Then the $K$-Toda bracket is the collection of all $\gamma \in \pi_{t+n-1}(X)$, where $\iota_{*}(\gamma)=g_{*}(\alpha)$.

We reconcile our use of the same notation in both definitions with the following lemma.

Lemma 4.4. The K-Toda bracket and the dual K-Toda bracket are equal.

Proof. Let $\alpha, f, g, \iota$, and $\nu$ be the maps given in our two definitions of the $K$-Toda bracket. For the purposes of this proof, we shall refer to the dual $K$-Toda bracket as $\langle K\rangle^{d}(\alpha)$. The map of cofiber sequences makes the following diagram commute:

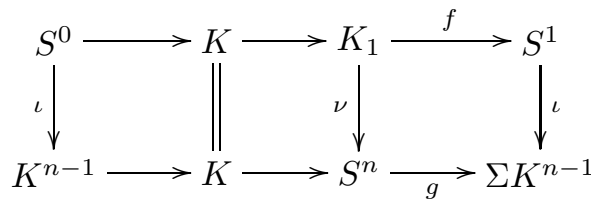

Taking the last square in the above triangle, extending it to a map of cofiber sequences the other way, and smashing with $X$, we get the following commutative diagram whose columns are exact:

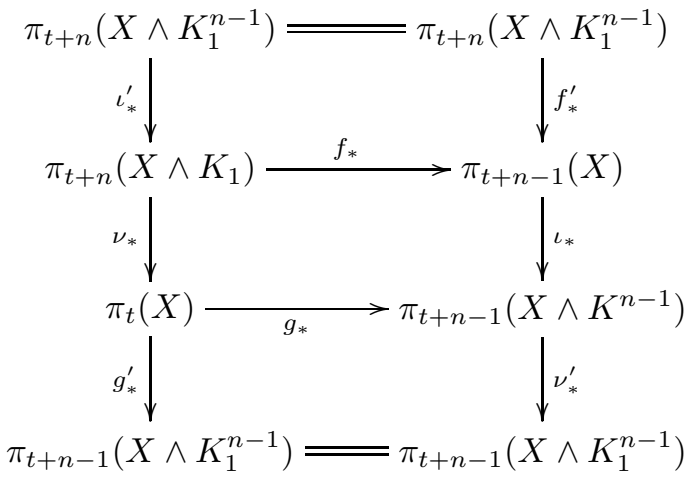

Here $\iota^{\prime}$ is the inclusion and $\nu^{\prime}$ is the projection, and $f^{\prime}$ and $g^{\prime}$ are determined from the diagram. The bracket $\langle K\rangle(\alpha)$ is computed by taking the preimage under $\nu_{*}$ and then applying $f_{*}$. The bracket $\langle K\rangle^{d}(\alpha)$ is computed by applying $g_{*}$ and then taking the preimage over $\iota_{*}$. We see that for any lift $\gamma \in \pi_{t+n}\left(X \wedge K_{1}\right)$ of $\alpha, f_{*}(\gamma)$ is a lift of $g_{*}(\alpha)$. It follows that we have $\langle K\rangle(\alpha) \subseteq\langle K\rangle^{d}(\alpha)$.

For the reverse containment, suppose that $\beta \in \pi_{t+n-1}(X)$ is a lift of $g_{*}(\alpha)$. Then $\beta$ is an arbitrary element of $\langle K\rangle^{d}(\alpha)$. Let $\gamma \in \pi_{t+n}\left(X \wedge K_{1}\right)$ be any lift of $\alpha$. Such 
a lift exists since

$$
g_{*}^{\prime}(\alpha)=\nu_{*}^{\prime} g_{*}(\alpha)=\nu_{*}^{\prime} \iota_{*}(\beta)=0 .
$$

We must add a correction term to $\gamma$ since it is not necessarily the case that $f_{*}(\gamma)=$ $\beta$. Let $\delta$ be the difference $f_{*}(\gamma)-\beta$. Then $\iota_{*}(\delta)=0$, so there is a lift to $\widetilde{\delta} \in$ $\pi_{t+n}\left(X \wedge K_{1}^{n-1}\right)$. Then $\gamma^{\prime}=\gamma-\iota_{*}^{\prime}(\widetilde{\delta})$ is another lift of $\alpha$, and $f_{*}(\gamma)=\beta$. We have therefore proven that $\langle K\rangle^{d}(\alpha) \subseteq\langle K\rangle(\alpha)$.

Remark 4.5. Perhaps a more conceptual way to prove Lemma 4.4 would be to regard the $K$-Toda bracket as a $d_{n}$ in the AHSS for $K$ with respect to the coskeletal filtration, and the dual $K$-Toda bracket as a $d_{n}$ in the AHSS with respect to the skeletal filtration. There is a comparison of these spectral sequences which is an isomorphism on $E_{1}$-terms, hence the spectral sequences are isomorphic, and in particular they have the same differentials $d_{n}$. See, for instance, Appendix B of [15].

We need to extend our $K$-Toda brackets to operations on the $E_{r}$ term of the $E$-ASS. Suppose that the attaching map $f: \Sigma^{-1} K_{1} \rightarrow S^{0}$ has $E$-Adams filtration $d$.

Definition 4.6 ( $K$-Toda brackets on $E_{r}(X)$ ). Suppose that $\alpha$ is an element of $E_{r}^{s, t}(X)$. Choose a lift of $\alpha$ to $\widetilde{\alpha} \in \pi_{t-s}\left(W_{s}^{s+r-1}(X)\right)$. Let $\widetilde{f}: \Sigma^{-1} K_{1} \rightarrow W_{d}\left(S^{0}\right)$ be a lift of $f$. Then we have

$$
\pi_{t-s}\left(W_{s}^{s+r-1}(X)\right) \stackrel{\nu_{*}}{\longleftarrow} \pi_{t-s+n}\left(W_{s}^{s+r-1}\left(X \wedge K_{1}\right)\right) \stackrel{\widetilde{f}_{*}}{\longrightarrow} \pi_{t+n-1}\left(W_{s+d}^{s+d+r-1}(X)\right) .
$$

If there exists a lift $\widetilde{\alpha}$ which is in the image of $\nu_{*}$, then we say that the $K$-Toda bracket is defined. Take $\gamma \in \pi_{t-s+n}\left(W_{s}^{s+r-1}(X)\right)$ such that $\nu_{*}(\gamma)=\widetilde{\alpha}$. Then the image of $\tilde{f}_{*}(\gamma)$ in $E_{r}^{s+d, t+d+n-1}(X)$ is in the $K$-Toda bracket $\langle K\rangle(\alpha)$. We define the $K$-Toda bracket to be the set of all such images for various choices of $\widetilde{\alpha}, \gamma$, and $\widetilde{f}$. We shall say that the $K$-Toda bracket has $E$-Adams degree $d$.

Remark 4.7. If the $K$-Toda bracket of $\alpha$ is defined, where $\alpha \in E_{\infty}^{s, t}(X)$, then if $\alpha$ detects $\bar{\alpha}$, every element of $\langle K\rangle(\alpha)$ detects an element of $\langle K\rangle(\bar{\alpha})$. In particular, if $\langle K\rangle(\alpha)$ contains 0 , then there is an element of $\langle K\rangle(\widetilde{\alpha})$ of $E$-Adams filtration greater than $k+d$.

\section{Statement of Results}

In this section we will state our main results concerning filtered root invariants. The proofs of many of the theorems are given in Section 6 . Throughout this section let $E$ be a ring spectrum, let $X$ be a finite complex, and suppose $\alpha$ is an element of $\pi_{t}(X)$ of $E$-bifiltration $(I, J)$ with

$$
\begin{aligned}
& I=\left(k_{1}, k_{2}, \ldots, k_{l}\right), \\
& J=\left(-N_{1},-N_{2}, \ldots,-N_{l}\right) .
\end{aligned}
$$

Theorem 5.1 (Relationship to homotopy root invariant). Suppose that $R_{E}^{\left[k_{i}\right]}(\alpha)$ contains a permanent cycle $\beta$. Then there exists an element $\bar{\beta} \in \pi_{*}(X)$ which $\beta$ detects such that the following diagram commutes up to elements of $E$-Adams 
filtration greater than or equal to $k_{i+1}$ :

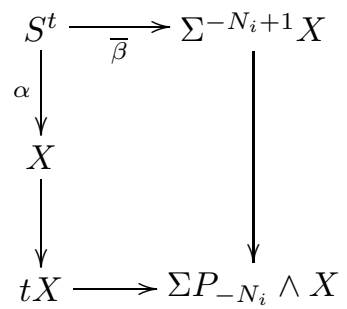

The proof of Theorem 5.1 is deferred to Section 6. If $k_{i+1}=\infty$, then $i$ is equal to $l$ (the maximal index of the bifiltration), and we get the following corollary.

Corollary 5.2. The top filtered root invariant $R_{E}^{\left[k_{l}\right]}(\alpha)$ is contained in $E_{\infty}(X)$. Let $\bar{R}_{E}^{\left[k_{l}\right]}(\alpha)$ be the set of elements of $\pi_{*}(X)$ detected by $R_{E}^{\left[k_{l}\right]}(\alpha)$. There are two possibilities:

(1) The image of the elements of $\bar{R}_{E}^{\left[k_{l}\right]}(\alpha)$ in $\pi_{*}\left(P_{-N_{l}} \wedge X\right)$ under the inclusion of the bottom cell is zero, and the homotopy root invariant lies in a higher stem than the $k_{l}^{\text {th }}$ filtered root invariant.

(2) There is an equality (modulo indeterminacy)

$$
\bar{R}_{E}^{\left[k_{l}\right]}(\alpha) \stackrel{\cap}{=}(\alpha) .
$$

Proof. Since $k_{l}=\infty$, the diagram in Theorem 5.1 commutes modulo elements of infinite Adams filtration. The $E$-Adams resolution was assumed to converge, so this means the diagram actually commutes. Let $\bar{\beta}$ be the element described in Theorem 5.1. If the image of $\bar{\beta}$ in $\pi_{t-1}\left(P_{-N_{i}}\right)$ is zero, then the homotopy root invariant lies in a higher stem than the $i^{\text {th }}$ filtered root invariant. Otherwise, $\bar{\beta}$ is an element of $R(\alpha)$.

Unfortunately, Corollary 5.2 is difficult to invoke in practice. This is because given a filtered root invariant, one usually does not know whether it is the highest one or not. In practice we are in a situation where we have a permanent cycle in a filtered root invariant, and we would like to show that the diagram of Theorem 5.1 commutes on the nose. One strategy is to write out the Atiyah-Hirzebruch spectral sequence for $\pi_{*}\left(P_{-N_{i}}\right)$ and try to show there are no elements of higher E-Adams filtration which could be the difference between the image of $\alpha$ and the image of $\bar{\beta}$ in $\pi_{*}\left(P_{-N_{i}}\right)$. This method is outlined in Procedure 9.1 .

We now present some theorems which relate filtered root invariants to differentials and compositions in the Adams spectral sequence. Recall that $r_{j}=k_{j+1}-k_{j}$.

Theorem 5.3 (Relationship to Adams differentials). Suppose that the $P_{-N_{i}}^{-N_{i+1}}$ Toda bracket has E-Adams degree $d$ and that $d \leq r_{i+1}$. Then the following is true:

(1) $\left\langle P_{-N_{i}}^{-N_{i+1}}\right\rangle\left(R_{E}^{\left[k_{i+1}\right]}(\alpha)\right)$ is defined and contains a permanent cycle.

(2) $R_{E}^{\left[k_{i}\right]}(\alpha)$ consists of elements which are $d_{r}$ cycles for $r<r_{i}+d$.

(3) There is a containment

$$
d_{r_{i}+d} R_{E}^{\left[k_{i}\right]}(\alpha) \subseteq\left\langle P_{-N_{i}}^{-N_{i+1}}\right\rangle\left(R_{E}^{\left[k_{i+1}\right]}(\alpha)\right)
$$

where both elements are thought of as elements of $E_{r_{i}+d}^{*, *}(X)$. 
The proof of Theorem 5.3 is deferred to Section 6 We intend to use Theorem 5.3 in reverse: given the $i^{\text {th }}$ filtered root invariant, we would like to deduce the $(i+1)^{\text {st }}$ filtered root invariant from the presence of an Adams differential. If the differential in Theorem 5.3 is zero, we still may be able to glean some information from the presence of a non-trivial composition.

Theorem 5.4 (Relationship to compositions). Suppose that $R_{E}^{\left[k_{i}\right]}(\alpha)$ is a coset of permanent cycles. Let $\widetilde{R}_{E}^{\left[k_{i}\right]}(\alpha)$ denote the coset of all lifts of elements of $R_{E}^{\left[k_{i}\right]}(\alpha)$ to $\pi_{t+N_{i}-1}\left(W_{k_{i}}(X)\right)$. When defined, there are lifts of the Toda brackets $\left\langle P_{-m}^{-N_{i}}\right\rangle\left(\widetilde{R}_{E}^{\left[k_{i}\right]}(\alpha)\right)$ to

$$
\left.\widetilde{\left\langle P_{-m}^{-N_{i}}\right.}\right\rangle\left(\widetilde{R}_{E}^{\left[k_{i}\right]}(\alpha)\right) \subseteq \pi_{t+m-2}\left(W_{k_{i+1}}(X)\right) .
$$

Let $M$ be the minimal such $m>N_{i}$ with the property that $\widetilde{\left\langle P_{-m}^{-N_{i}}\right\rangle}\left(\widetilde{R}_{E}^{\left[k_{i}\right]}(\alpha)\right)$ contains a non-trivial element. Let $d$ be the E-Adams degree of the Toda bracket $\left\langle P_{-N_{i}}^{-N_{i+1}}\right\rangle(-)$, and suppose that $d \leq r_{i+1}$. Then the following are true:

(1) Let

$$
\bar{R}_{E}^{\left[k_{i}\right]}(\alpha) \subseteq \pi_{t+N_{i}-1}(X)
$$

denote the elements which are detected by the permanent cycles of $R_{E}^{\left[k_{i}\right]}(\alpha)$. Then the Toda bracket

$$
\left\langle P_{-M}^{-N_{i}}\right\rangle\left(\bar{R}_{E}^{\left[k_{i}\right]}(\alpha)\right) \subseteq \pi_{t+M-2}(X)
$$

is defined.

(2) The Toda bracket $\left\langle P_{-M}^{-N_{i+1}}\right\rangle\left(R_{E}^{\left[k_{i+1}\right]}(\alpha)\right)$ is defined in $E_{r_{i+1}}(X)$, and contains a permanent cycle. We shall denote the collection of all elements which are detected by these permanent cycles by

$$
\overline{\left\langle P_{-M}^{-N_{i+1}}\right\rangle\left(R_{E}^{\left[k_{i+1}\right]}(\alpha)\right)} \subseteq \pi_{t+M-2}(X) .
$$

(3) There is an equality (modulo indeterminacy)

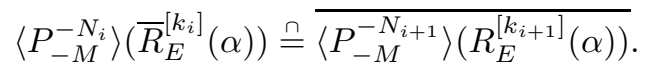

Remark 5.5. The hypothesis $d \leq r_{i+1}$ in Theorems 5.3 and 5.4 is a necessary technical hypothesis to make the proofs work. In practice, $d$ is often equal to 1. Since $r_{j}$ is always positive, the hypothesis is satisfied in this case.

Finally, we give a partial description of the first filtered root invariant. We begin with a simple observation.

Lemma 5.6. If filt $_{E}(\alpha)=k$, then $R_{E}^{[s]}(\alpha)$ is trivial for $s<k$.

Proof. We must show that in the $E$-bifiltration of $\alpha, k_{1} \geq k$. Consider the following diagram:

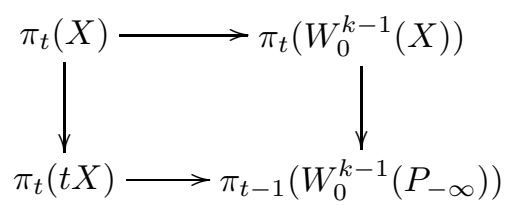

Since $\operatorname{filt}_{E}(\alpha)=k$, the image of $\alpha$ in $\pi_{t}\left(W_{0}^{k-1}(X)\right)$ is trivial. Therefore the image of $\alpha$ in $\pi_{t-1}\left(W_{0}^{k-1}\left(P_{-\infty}\right)\right)$ is trivial. By the maximality of $k_{1}$, we have $k_{1} \geq k$. 
If filt $_{E}(\alpha)=0$ (i.e. when $\alpha$ has a non-trivial Hurewicz image), then we can sometimes identify the first non-trivial filtered root invariant with the $E$-root invariant.

Proposition 5.7 (Relationship to the E-root invariant). The E-root invariant $R_{E}(\alpha)$ is non-trivial if and only if $R_{E}^{[0]}(\alpha)$ is non-trivial. If this is the case, regarding $R_{E}(\alpha)$ as being contained in $E_{1}^{0, *}(X)$, we have

$$
R_{E}^{[0]}(\alpha) \subseteq R_{E}(\alpha)
$$

Proof. This is immediate from the definitions.

If filt $_{E}(\alpha)=1$, then the $E$-root invariant is trivial and Lemma 5.6] implies that the zeroth filtered root invariant is trivial. We can however sometimes compute the first filtered root invariant using the $E \wedge \bar{E}$-root invariant.

Proposition 5.8 (Relationship to the $E \wedge \bar{E}$-root invariant). Suppose that $\alpha$ has $E$ Adams filtration 1. Then there exists an element $\widetilde{\alpha} \in \pi_{t}\left(E \wedge \bar{E} \wedge X_{p}^{\wedge}\right)=E_{1}^{1, t+1}\left(X_{p}^{\wedge}\right)$ which detects $\alpha$ in the $E-A S S$, and such that $R_{E \wedge}(\widetilde{\alpha})$ is trivial if and only if $R_{E}^{[1]}(\alpha)$ is trivial. There is a containment

$$
R_{E}^{[1]}(\alpha) \subseteq R_{E \wedge \bar{E}}(\widetilde{\alpha}) .
$$

In practice, we will not know which choice of detecting element $\widetilde{\alpha}$ to choose, so the following corollary will prove useful.

Corollary 5.9. Suppose that filt f $_{E}(\alpha)=1$. If $\widetilde{\alpha} \in \pi_{t}(E \wedge \bar{E} \wedge X)$ is any element which detects $\alpha$ in the E-ASS, then

$$
R_{E}^{[1]}(\alpha) \subseteq R_{E \wedge \bar{E}}(\widetilde{\alpha})+A
$$

Here $A$ is the image of the map

$$
\pi_{t}\left(W_{0}^{(0,1)}\left(P_{(-N,-N+1)} \wedge X\right)\right) \stackrel{\partial}{\rightarrow} \pi_{t-1}\left(W_{1}^{1}\left(\Sigma^{-N} X\right)\right),
$$

where $\partial$ is the boundary homomorphism associated to the cofiber sequence

$$
W_{1}^{1}\left(\Sigma^{-N} X\right) \stackrel{\iota}{\rightarrow} W_{0}^{1}\left(P_{-N} \wedge X\right) \rightarrow W_{0}^{(0,1)}\left(P_{(-N,-N+1)} \wedge X\right)
$$

and the $E \wedge \bar{E}$-root invariant of $\widetilde{\alpha}$ is carried by the $-N$-cell of $P_{-\infty}$.

The proof of Proposition 5.8 and Corollary 5.9] is deferred to Section 6. When $E=H \mathbb{F}_{p}=H$, we can identify the first filtered root invariant with the algebraic root invariant.

Theorem 5.10 (Relationship to the algebraic root invariant). If $E$ is the EilenbergMac Lane spectrum $H \mathbb{F}_{p}=H$ and $\alpha$ has Adams filtration $k$, then $k_{1}=k$. Furthermore, the filtered root invariant $R_{H}^{[k]}(\alpha)$ consists of $d_{1}$ cycles which detect a coset of non-trivial elements $\bar{R}_{H}^{[k]}(\alpha) \subseteq E_{2}^{k, t+k+N_{1}-1}(X)$, and there exists a choice of $\widetilde{\alpha} \in E_{2}^{k, t+k}(X)$ which detects $\alpha$ in the ASS such that

$$
\bar{R}_{H}^{[k]}(\alpha) \subseteq R_{a l g}(\widetilde{\alpha})
$$

The proof of Theorem 5.10 is deferred to Section 6 . We have given a partial scenario as to how the filtered root invariants can be used to calculate root invariants using the $E$-ASS. One first calculates the zeroth filtered root invariant as an $E$-root invariant or the first non-trivial root invariant as an algebraic root invariant. Then 
the idea, while only sometimes correct, is that "if a filtered root invariant does not detect the root invariant, then it either supports a differential or a composition that points to the next filtered root invariant." The last filtered root invariant then has a chance of detecting the homotopy root invariant. We stress that many things can interfere with this actually happening.

\section{Proofs of the main theorems}

In all of the proofs below, we shall assume that our finite complex $X$ is actually $S^{0}$. The general case is no different, but smashing everything with $X$ complicates the notation.

Proof of Theorem 5.1. Let $\beta$ be an element of $R_{E}^{\left[k_{i}\right]}(\alpha)$. Then there exists a lift $f^{\alpha}$ such that $\beta$ is the image of $f^{\alpha}$ under the collapse map

$$
\pi_{t-1}\left(W_{I}\left(P^{J}\right)\right) \rightarrow \pi_{t-1}\left(W_{k_{i}}^{k_{i+1}-1}\left(S^{-N_{i}}\right)\right) .
$$

Consider the following diagram:

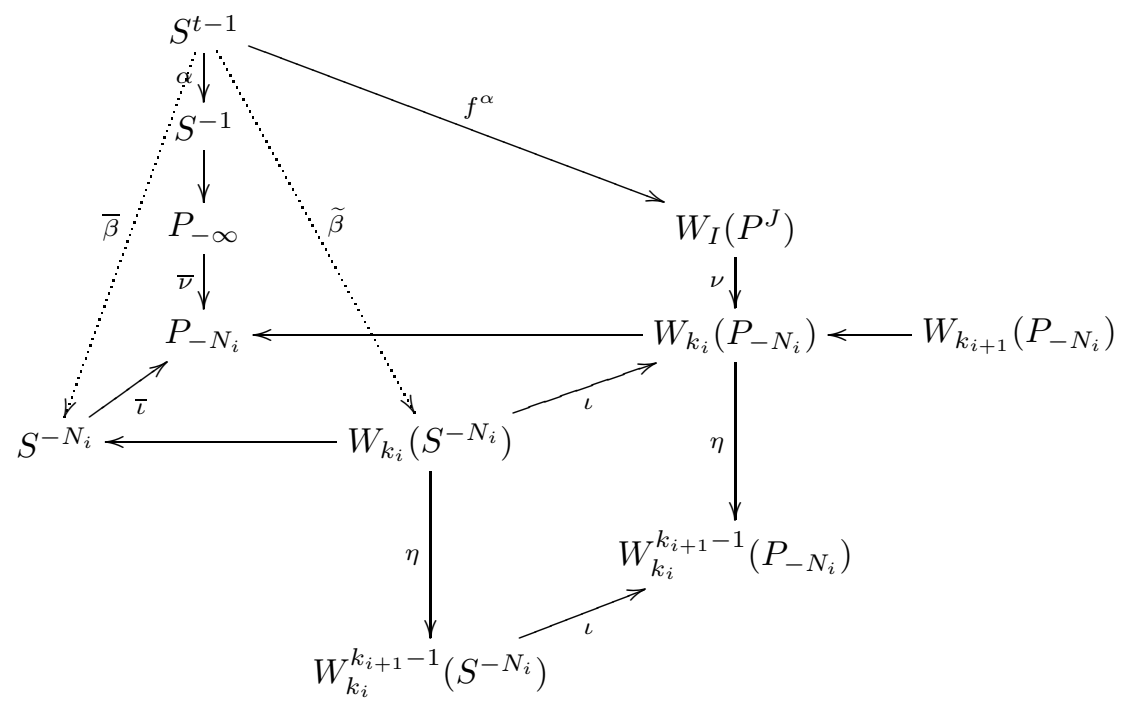

In the above diagram, $\nu$ is induced from the composite

$$
W_{I}\left(P^{J}\right) \rightarrow W_{I^{\prime}}\left(P_{-N_{i}}^{J^{\prime}}\right) \rightarrow W_{k_{i}}\left(P_{-N_{i}}\right)
$$

where $I^{\prime}=\left(k_{i}, \ldots, k_{l}\right)$ and $J^{\prime}=\left(-N_{i}, \ldots,-N_{l}\right)$. Since $R_{E}^{\left[k_{i}\right]}(\alpha)$ contains a permanent cycle $\beta$, there exists a map $\widetilde{\beta}$ (as above) such that $\eta \widetilde{\beta}$ projects to $\beta$, and such that $\iota \eta \widetilde{\beta}=\eta \nu f^{\alpha}$. If $\delta=\iota \widetilde{\beta}-\nu f^{\alpha}$, then $\delta$ lifts to $\pi_{t-1}\left(W_{k_{i+1}}\left(P_{-N_{i}}\right)\right)$. Let $\bar{\beta}$ be the map induced by $\widetilde{\beta}$, and denote by $\bar{\delta} \in \pi_{t-1}\left(P_{-N_{i}}\right)$ the image of $\delta$. Then filt $_{E}(\bar{\delta}) \geq k_{i+1}$ and we have the following formula:

$$
\bar{\iota} \circ \bar{\beta}=\bar{\nu} \circ \alpha+\bar{\delta} .
$$

This is precisely what we wanted to prove.

Proof of Theorem 5.3. Fix a lift $f^{\alpha} \in \pi_{t-1}\left(W_{I}\left(P^{J}\right)\right)$ of $\alpha$. Define a spectrum

$$
U=W_{\left(k_{i}, k_{i+1}, k_{i+2}\right)}\left(P_{-N_{i}}^{\left(-N_{i},-N_{i+1}, \infty\right)}\right) .
$$


There is a natural map

$$
W_{I}\left(P^{J}\right) \rightarrow U
$$

and let $\gamma \in \pi_{t-1}(U)$ be the image of $f^{\alpha}$ under this map.

Since we have assumed that the $P_{-N_{i}}^{-N_{i+1}}$-Toda bracket has E-Adams degree $d$, there is a lift of the attaching map

$$
f: \Sigma^{-1} P_{-N_{i}+1}^{-N_{i+1}} \rightarrow S^{-N_{i}}
$$

to a map

$$
\tilde{f}: \Sigma^{-1} P_{-N_{i}+1}^{-N_{i+1}} \rightarrow W_{d}\left(S^{-N_{i}}\right) .
$$

Define a filtered stunted projective space $\left(P_{-N_{i}}^{-N_{i+1}}\right)_{[d]}$ by the following cofiber sequence:

$$
\Sigma^{-1} P_{-N_{i}+1}^{-N_{i+1}} \stackrel{\tilde{f}}{\rightarrow} W_{d}\left(S^{-N_{i}}\right) \rightarrow\left(P_{-N_{i}}^{-N_{i+1}}\right)_{[d]} .
$$

The spectrum $U$ is given by the homotopy pushout

$$
\left(W_{k_{i+2}}\left(P_{-N_{i}}\right) \sqcup_{W_{k_{i+2}}\left(P_{-N_{i}}^{-N_{i+1}}\right)} W_{k_{i+1}}\left(P_{-N_{i}}^{-N_{i+1}}\right)\right) \sqcup_{W_{k_{i+1}}\left(S^{-N_{i}}\right)} W_{k_{i}}\left(S^{-N_{i}}\right) .
$$

Since we have assumed that $d \leq r_{i+1}=k_{i+2}-k_{i+1}$, the spectrum $U$ admits the equivalent description as

$$
\left(W_{k_{i+2}}\left(P_{-N_{i}}\right) \sqcup_{W_{k_{i+2}}\left(P_{-N_{i}}^{-N_{i+1}}\right)} W_{k_{i+1}}\left(\left(P_{-N_{i}}^{-N_{i+1}}\right)_{[d]}\right)\right) \sqcup_{W_{k_{i+1}+d}\left(S^{-N_{i}}\right)} W_{k_{i}}\left(S^{-N_{i}}\right) .
$$

Consider the following commutative diagram:

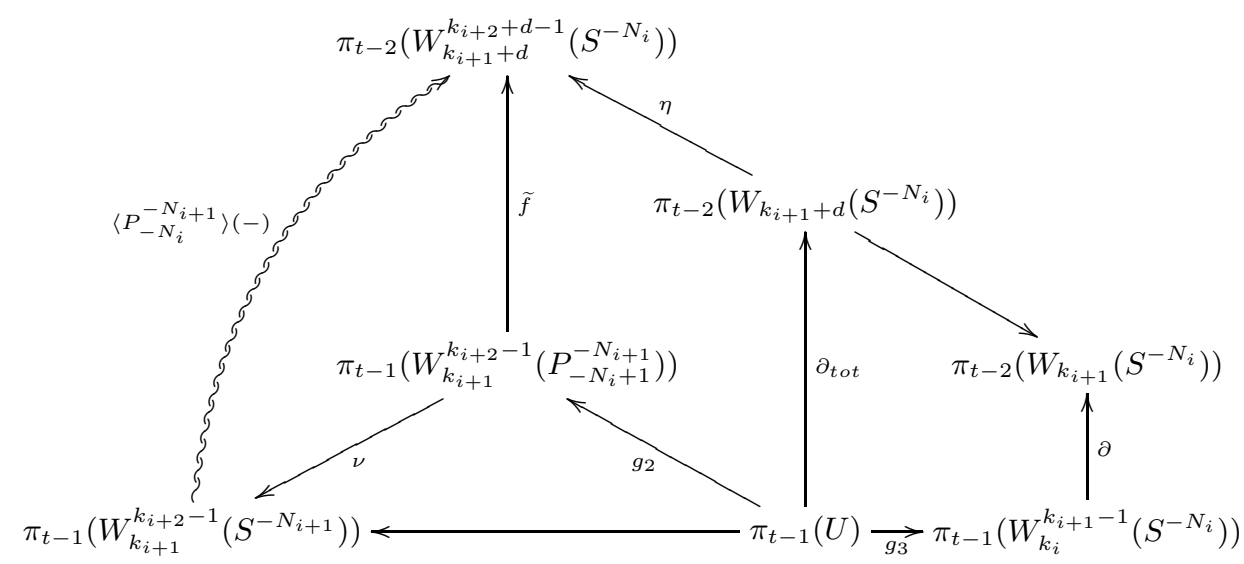

Here $g_{3}$ is obtained by collapsing out the first and second factors of the homotopy pushout $U$, and similarly $g_{2}$ is obtained by collapsing out the first and third factors. The map $\partial_{t o t}$ is the boundary homomorphism of the Meyer-Vietoris sequence, and may be thought of as collapsing out all of the factors of the homotopy pushout. The wavy arrow indicates that the $P_{-N_{i}}^{-N_{i+1}}$-Toda bracket is taken by taking the inverse image under $\nu$, followed by application of $\tilde{f}$.

Our element $\gamma \in \pi_{t-1}(U)$ has compatible images in all of the other groups in the diagram. The image of $\gamma$ under $g_{3}$ projects to an element of $R_{E}^{\left[k_{i}\right]}(\alpha)$ in $E_{r_{i}}$. Following the outside of the diagram from the lower right-hand corner to the top corner counter-clockwise amounts to taking $d_{r_{i}+d}$ in the E-ASS. Following from the lower right-hand corner to the top clockwise applies the Toda bracket to the 
$k_{i+1}^{\text {st }}$ filtered root invariant. Thus $d_{r_{i}+d} R_{E}^{\left[k_{i}\right]}(\alpha)$ and $\left\langle P_{-N_{i}}^{-N_{i+1}}\right\rangle\left(R_{E}^{[k i+1]}(\alpha)\right)$ have a common element.

Proof of Theorem 5.4. Fix a lift $f^{\alpha} \in \pi_{t-1}\left(W_{I}\left(P^{J}\right)\right)$ of $\alpha$. Let $V_{m}$ be defined by

$$
V_{m}=W_{\left(0, k_{i}, k_{i+1}, k_{i+2}\right)}\left(P_{-m}^{\left(-N_{i-1},-N_{i},-N_{i+1}, \infty\right)}\right) .
$$

Then $V_{m}$ is defined by the following homotopy pushout:

$$
\begin{gathered}
P_{-m}^{-N_{i-1}} \sqcup_{W_{k_{i}}\left(P_{-m}^{-N_{i-1}}\right)} W_{k_{i}}\left(P_{-m}^{-N_{i}}\right) \sqcup_{W_{k_{i+1}}\left(P_{-m}^{-N_{i}}\right)} \\
W_{k_{i+1}}\left(P_{-m}^{-N_{i+1}}\right) \sqcup_{W_{k_{i+2}}\left(P_{-m}^{-N_{i+1}}\right)} W_{k_{i+2}}\left(P_{-m}\right) .
\end{gathered}
$$

There is a natural map

$$
W_{I}\left(P^{J}\right) \rightarrow V_{m}
$$

and we define $\gamma_{m} \in \pi_{t-1}\left(V_{m}\right)$ to be the image of $f^{\alpha}$ under this map. We need to lift $\gamma_{m}$ a little more. The composite

$$
\pi_{t-1}\left(V_{m}\right) \stackrel{\partial}{\rightarrow} \pi_{t-2}\left(W_{k_{i+1}}\left(P_{-m}^{-N_{i}}\right)\right) \rightarrow \pi_{t-2}\left(W_{k_{i+1}}\left(S^{-N_{i}}\right)\right)
$$

sends $\gamma_{m}$ to zero, since it carries the $E$-Adams differential of an element in $R_{E}^{\left[k_{i}\right]}(\alpha)$, which was hypothesized to be zero. Here $\partial$ is a Meyer-Vietoris boundary homomorphism. Thus our element $\gamma_{m}$ lifts to an element $\widetilde{\gamma}_{m} \in \pi_{t-1}\left(\widetilde{V}_{m}\right)$, where $\widetilde{V}_{m}$ is the following spectrum:

$$
\begin{gathered}
P_{-m}^{-N_{i-1}} \sqcup_{W_{k_{i}}\left(P_{-m}^{-N_{i-1}}\right)} W_{k_{i}}\left(P_{-m}^{-N_{i}}\right) \sqcup_{W_{k_{i+1}}\left(P_{-m}^{-N_{i}-1}\right)} \\
W_{k_{i+1}}\left(P_{-m}^{-N_{i+1}}\right) \sqcup_{W_{k_{i+2}}\left(P_{-m}^{-N_{i+1}}\right)} W_{k_{i+2}}\left(P_{-m}\right) .
\end{gathered}
$$

The following diagram explains the lifted bracket $\widetilde{\left\langle P_{-m}^{-N_{i}}\right\rangle}\left(\widetilde{R}_{E}^{\left[k_{i}\right]}(\alpha)\right)$ :

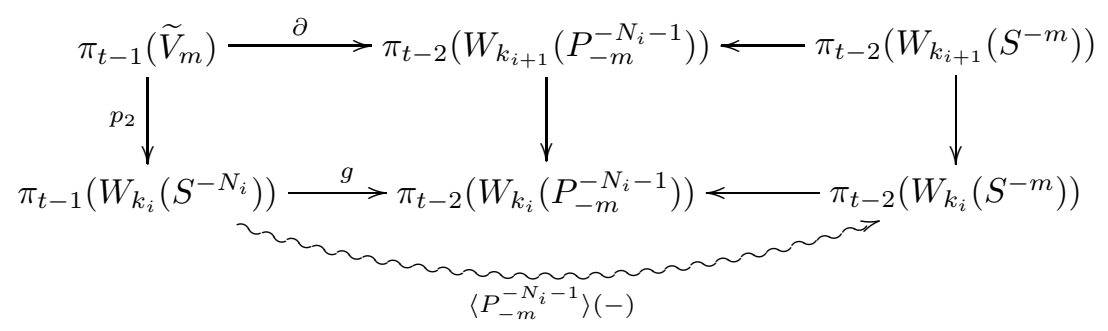

Here $p_{2}$ is projection onto the second factor of the homotopy pushout $\widetilde{V}_{m}$, $\partial$ is a Meyer-Vietoris boundary, and $g$ is the attaching map of the $-N_{i}$-cell to $P_{-m}^{-N_{i}-1}$. Assuming (inductively) that for every $N_{i}<m^{\prime}<m$

$$
\left.\widetilde{\left\langle P_{-m^{\prime}}^{-N_{i}}\right.}\right\rangle\left(\widetilde{R}_{E}^{\left[k_{i}\right]}(\alpha)\right)=0
$$

there is a lift of $\partial\left(\widetilde{\gamma}_{m}\right)$ to $\pi_{t-2}\left(W_{k_{i+1}}\left(S^{-m}\right)\right.$. The set of all such lifts is defined to be

$$
\widetilde{\left\langle P_{-m}^{-N_{i}}\right\rangle}\left(\widetilde{R}_{E}^{\left[k_{i}\right]}(\alpha)\right) \subseteq \pi_{t-2}\left(W_{k_{i+1}}\left(S^{-m}\right)\right) .
$$

Diagram (6.1) implies that this set of lifts is indeed a lift of the Toda bracket

$$
\left\langle P_{-m}^{-N_{i}}\right\rangle\left(\widetilde{R}_{E}^{\left[k_{i}\right]}(\alpha)\right) \subseteq \pi_{t-2}\left(W_{k_{i}}\left(S^{-m}\right)\right) .
$$


Let $M$ be the first $m$ such that $\widetilde{\left\langle P_{-m}^{-N_{i}}\right\rangle}\left(\widetilde{R}_{E}^{\left[k_{i}\right]}(\alpha)\right)$ contains a non-trivial element. Let $\widetilde{\gamma}=\widetilde{\gamma}_{M}$ and let $\widetilde{V}=\widetilde{V}_{M}$. The image of $\widetilde{\gamma}$ under the composite

$$
\pi_{t-1}(\widetilde{V}) \stackrel{\partial}{\rightarrow} \pi_{t-2}\left(W_{k_{i+1}}\left(P_{-M}^{-N_{i}-1}\right)\right) \rightarrow \pi_{t-2}\left(W_{k_{i+1}}\left(P_{-M+1}^{-N_{i}-1}\right)\right)
$$

is zero, and $\widetilde{\gamma}$ lifts even further, to an element $\widetilde{\widetilde{\gamma}} \in \pi_{t-1}(\widetilde{\widetilde{V}})$, where $\widetilde{\widetilde{V}}$ is defined to be the following spectrum:

$$
\begin{gathered}
P_{-M}^{-N_{i-1}} \sqcup_{W_{k_{i}}\left(P_{-M}^{-N_{i-1}}\right)} W_{k_{i}}\left(P_{-M}^{-N_{i}}\right) \sqcup_{W_{k_{i+1}}\left(S^{-M}\right)} \\
W_{k_{i+1}}\left(P_{-M}^{-N_{i+1}}\right) \sqcup_{W_{k_{i+2}}\left(P_{-M}^{-N_{i+1}}\right)} W_{k_{i+2}}\left(P_{-M}\right) .
\end{gathered}
$$

Let $f$ be the attaching map

$$
f: \Sigma^{-1} P_{-M+1}^{-N_{i+1}} \rightarrow S^{-M}
$$

Since $\left\langle P_{-M}^{-N_{i+1}}\right\rangle$ has $E$-Adams degree $d$, there is a lift

$$
\tilde{f}: \Sigma^{-1} P_{-M+1}^{-N_{i+1}} \rightarrow W_{d}\left(S^{-M}\right) .
$$

Let $\left(P_{-M}^{-N_{i+1}}\right)_{[d]}$ be the cofiber of $\tilde{f}$. Our hypothesis that $d \leq r_{i+1}$ implies that $\widetilde{\widetilde{V}}$ has the following equivalent description as a homotopy pushout:

$$
\begin{aligned}
& P_{-M}^{-N_{i-1}} \sqcup_{W_{k_{i}}\left(P_{-M}^{-N_{i-1}}\right)} W_{k_{i}}\left(P_{-M}^{-N_{i}}\right) \sqcup_{W_{k_{i+1}+d}\left(S^{-M}\right)} \\
& W_{k_{i+1}}\left(\left(P_{-M}^{-N_{i+1}}\right)_{[d]}\right) \sqcup_{W_{k_{i+2}}\left(P_{-M}^{-N_{i+1}}\right)} W_{k_{i+2}}\left(P_{-M}\right) .
\end{aligned}
$$

Our proof is reduced to chasing the diagram displayed in Figure 3 ,

In Figure 3, $\widetilde{f}$ and $g$ are attaching maps, $p_{2}$ is a projection onto the second factor of the homotopy pushout $\widetilde{\widetilde{V}}, p_{3}$ is a projection onto the third factor followed by a projection onto the $-N_{i}$-cell, and $\partial$ is the Meyer-Vietoris boundary. The wavy arrows represent Toda brackets. The element $\widetilde{\widetilde{\gamma}} \in \widetilde{\widetilde{V}}$ maps to compatible elements of every group displayed in Figure 3 The image of $\widetilde{\widetilde{\gamma}}$ in $\pi_{t-1}\left(W_{k_{i}}^{k_{i+1}-1}\left(S^{-N_{i}}\right)\right)$ projects to an element of $R_{E}^{\left[k_{i}\right]}(\alpha)$. The image of $\widetilde{\widetilde{\gamma}}$ in $\pi_{t-1}\left(W_{k_{i+1}}^{k_{i+2}-1}\left(S^{-N_{i+1}}\right)\right)$ projects to an element of $R_{E}^{\left[k_{i+1}\right]}(\alpha)$. Let $\beta$ be the image of $\widetilde{\widetilde{\gamma}}$ in $\pi_{t-2}\left(S^{-M}\right)$. Following the outside of Figure 3 from the lower left-hand corner clockwise reveals that

$$
\beta \in \overline{\left\langle P_{-M}^{-N_{i+1}}\right\rangle\left(R_{E}^{\left[k_{i+1}\right]}(\alpha)\right)} .
$$

Following the outside of Figure 3 from the lower right-hand corner counterclockwise gives

$$
\beta \in\left\langle P_{-M}^{-N_{i}}\right\rangle\left(\bar{R}_{E}^{\left[k_{i}\right]}(\alpha)\right) .
$$

Thus $\beta$ is a common element of the two groups.

Proof of Proposition 5.8. Suppose that the first filtered root invariant of $\alpha$ is carried by the $-N$-cell of $P_{-\infty}$. If the first filtered root invariant is trivial, then $N=\infty$. 


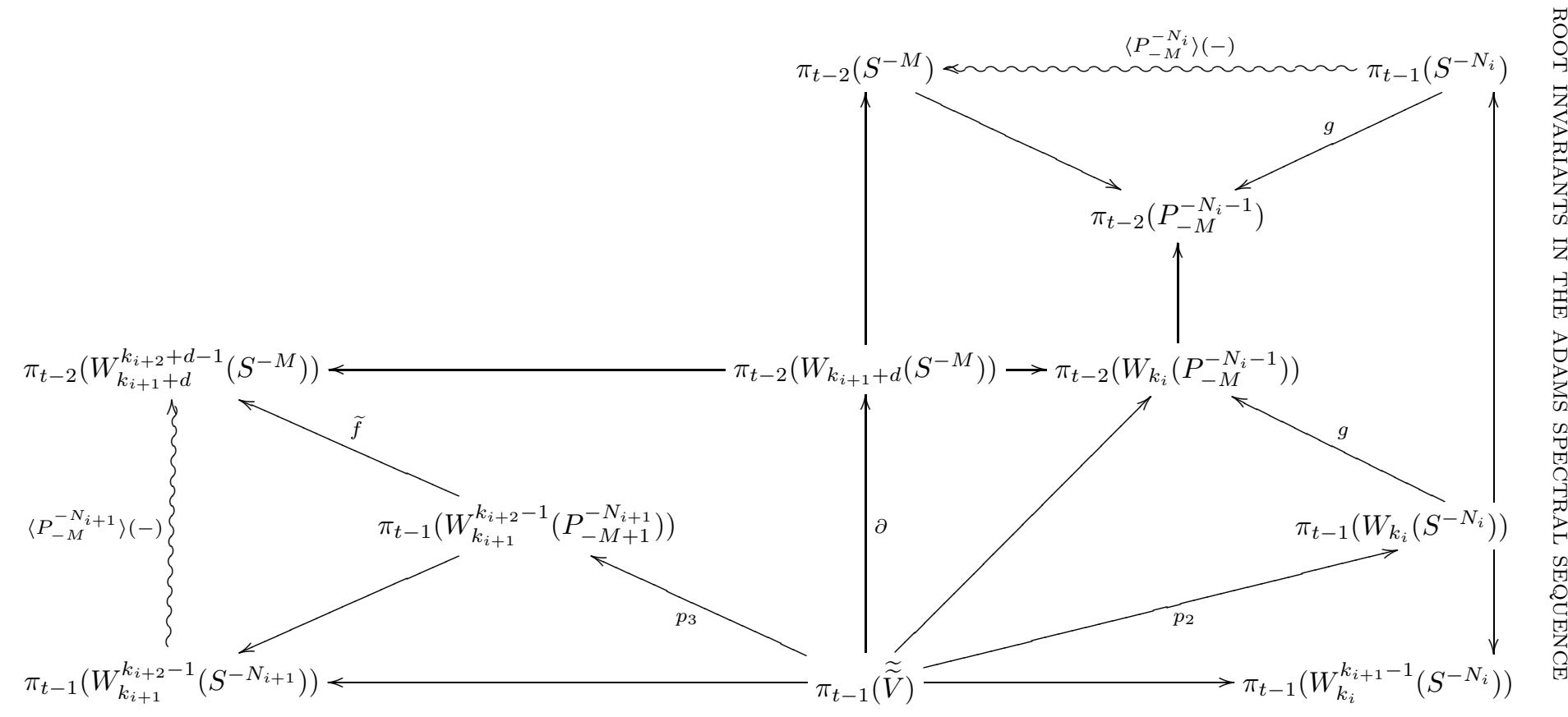

Figure 3. The main diagram for the proof of Theorem 5.4 
Consider the following diagram:

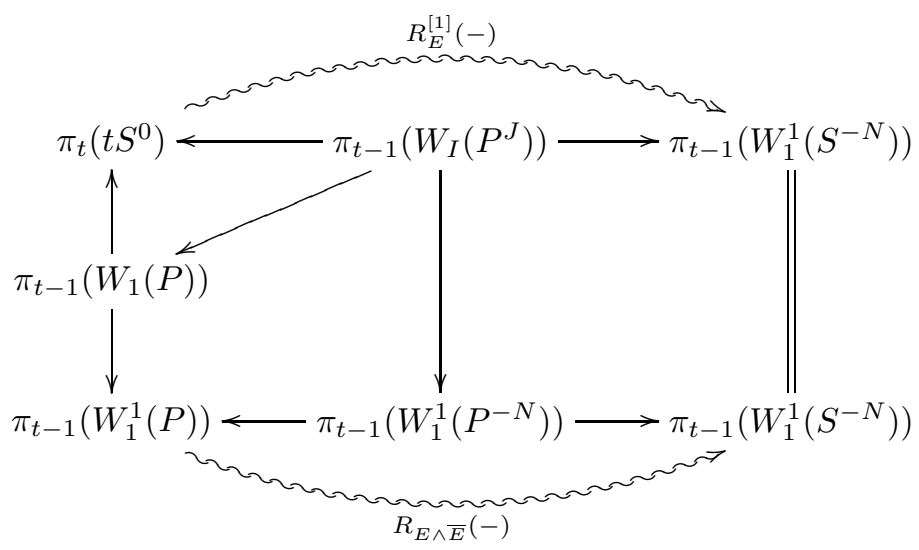

Let $f^{\alpha}$ be a lift of the image of $\alpha$ in $\pi_{t-1}\left(t S^{0}\right)$ to $\pi_{t-1}\left(W_{I}\left(P^{J}\right)\right)$. Then $f^{\alpha}$ has compatible images in every group in the diagram. The detecting element $\widetilde{\alpha}$ is the image of $f^{\alpha}$ in $\pi_{t-1}\left(W_{1}^{1}\left(P_{-\infty}\right)\right)=\pi_{t-1}\left(\Sigma^{-1} E \wedge \bar{E}_{p}^{\wedge}\right)$.

Proof of Corollary [5.9. First observe that if the first filtered root invariant is carried by the $-M$-cell, then $M \geq N$. Let $\widetilde{\alpha}^{\prime}$ be a 'preferred' detecting element such as the one described in Proposition 5.8. It suffices to show that if $\beta \in R_{E \wedge}(\widetilde{\alpha})$ and $\beta^{\prime} \in R_{E \wedge \bar{E}}\left(\widetilde{\alpha}^{\prime}\right)$ (or $\beta^{\prime}=0$ if $R_{E \wedge \bar{E}}\left(\widetilde{\alpha}^{\prime}\right)$ lies in a higher degree than $R_{E \wedge \bar{E}}(\widetilde{\alpha})$ ), then $\beta-\beta^{\prime} \in A$. The elements $\beta$ and $\beta^{\prime}$ are the images of elements $\gamma$ and $\gamma^{\prime}$, respectively, under the collapse map

$$
\pi_{t-1}\left(W_{1}^{1}\left(P^{-N}\right)\right) \rightarrow \pi_{t-1}\left(W_{1}^{1}\left(S^{-N}\right)\right),
$$

where $\gamma$ and $\gamma^{\prime}$ both map to the image of $\alpha$ under the homomorphism

$$
\pi_{t-1}\left(W_{1}^{1}\left(P^{-N}\right)\right) \stackrel{\iota}{\rightarrow} \pi_{t-1}\left(W_{0}^{1}(P)\right) .
$$

Therefore there is an element $\mu \in \pi_{t}\left(W_{0}^{(0,1)}\left(P_{(-\infty,-N+1)}\right)\right)$ such that $\gamma-\gamma^{\prime}=\partial_{1}(\mu)$, where $\partial_{1}$ is the connecting homomorphism of the long exact sequence associated to the cofiber sequence

$$
W_{1}^{1}\left(P^{-N}\right) \rightarrow W_{0}^{1}(P) \rightarrow W_{0}^{(0,1)}\left(P_{(-\infty,-N+1)}\right) .
$$

A comparison of cofiber sequences shows that there is a commutative diagram

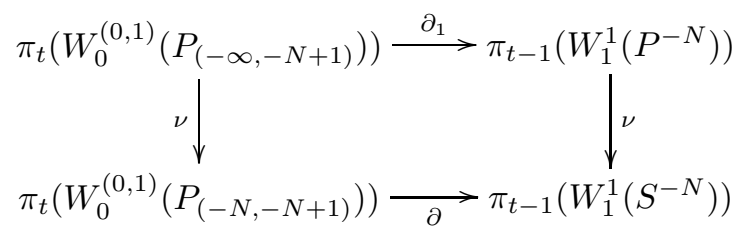

Thus we see that

$$
\beta-\beta^{\prime}=\nu\left(\gamma-\gamma^{\prime}\right)=\nu \circ \partial_{1}(\mu)=\partial \circ \nu(\mu),
$$

so $\beta-\beta^{\prime}$ is contained in $A$.

Proof of Theorem 5.10. We shall refer to the appropriate Ext groups as the $E_{2}$ terms of the ASS. Observe that Lemma 5.6 implies that $k_{1} \geq k$. We first will prove 
that $k_{1}=k$. Suppose that $k_{1}>k$. Let $\widetilde{\widetilde{\alpha}}$ be a lift of $\widetilde{\alpha}$ to $\pi_{t}\left(W_{k}\left(S^{0}\right)\right)$. Consider the following diagram.

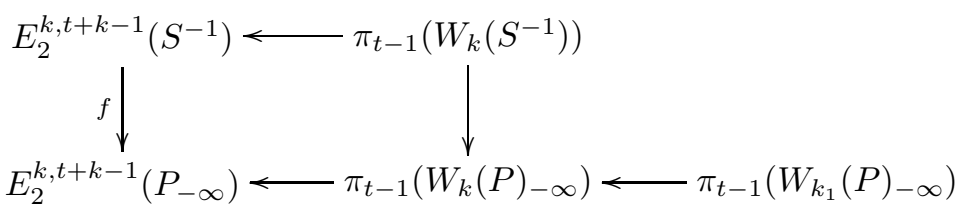

The element $\widetilde{\widetilde{\alpha}}$ maps to $\widetilde{\alpha} \in E_{2}^{k, t+k-1}\left(S^{-1}\right)$. By the definition of $k_{1}$, the image of $\widetilde{\widetilde{\alpha}}$ in $\pi_{t-1}\left(W_{k}(P)_{-\infty}\right)$ lifts to an element of $\pi_{t-1}\left(W_{k_{1}}(P)_{-\infty}\right)$. This implies that $f(\widetilde{\alpha})=0$. However, $\widetilde{\alpha}$ is non-zero, and the algebraic Segal conjecture implies that $f$ is an isomorphism. We conclude that $k_{1}=k$.

The filtered root invariant $R_{H}^{[1]}(\alpha)$ is a subset of $E_{1}^{k, t+N_{1}+k-1}\left(S^{0}\right)$. All of the attaching maps of $P_{-\infty}$ are of positive Adams filtration, so Theorem 5.3 implies that $R_{E}^{[1]}(\alpha)$ consists of $d_{1}$-cycles. Let $f^{\alpha} \in \pi_{t-1}\left(W_{I}\left(P^{J}\right)\right)$ be a lift of $\alpha$, and let $\beta \in \pi_{t-1}\left(W_{k}^{k}\left(S^{-N_{1}}\right)\right)$ be the corresponding element in $R_{H}^{[k]}(\alpha)$. Let $f_{1}^{\alpha} \in \pi_{t-1}\left(W_{k}(P)_{-\infty}\right)$ be the image of $f^{\alpha}$ under the natural map

$$
W_{I}\left(P^{J}\right) \rightarrow W_{k}(P)_{-\infty}
$$

Consulting the diagram,

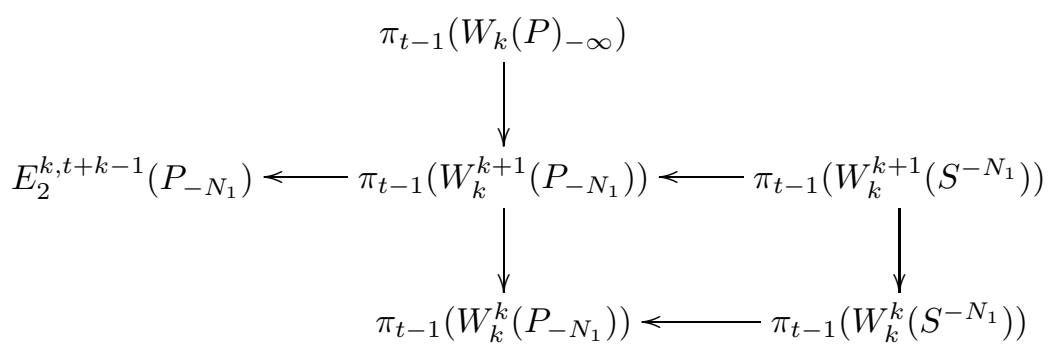

both $f_{1}^{\alpha}$ and $\beta$ have the same image in $\pi_{t-1}\left(W_{k}^{k}\left(P_{-N_{1}}\right)\right)$. Let $\gamma$ be the image of $f_{1}^{\alpha}$ in $\pi_{t-1}\left(W_{k}^{k+1}\left(P_{-N_{1}}\right)\right)$. Since $\beta$ is a $d_{1}$-cycle, it lifts to $\widetilde{\beta} \in \pi_{t-1}\left(W_{k}^{k+1}\left(S^{-N_{1}}\right)\right)$. Let $\widetilde{\gamma}$ be the image of $\widetilde{\beta}$ in $\pi_{t-1}\left(W_{k}^{k+1}\left(P_{-N_{1}}\right)\right)$. It is not necessarily the case that $\gamma=\widetilde{\gamma}$, but it is the case that the difference $\gamma-\widetilde{\gamma}$ vanishes in $E_{2}^{k, t+k-1}\left(P_{-N_{1}}\right)$. 
Consider the following diagram:

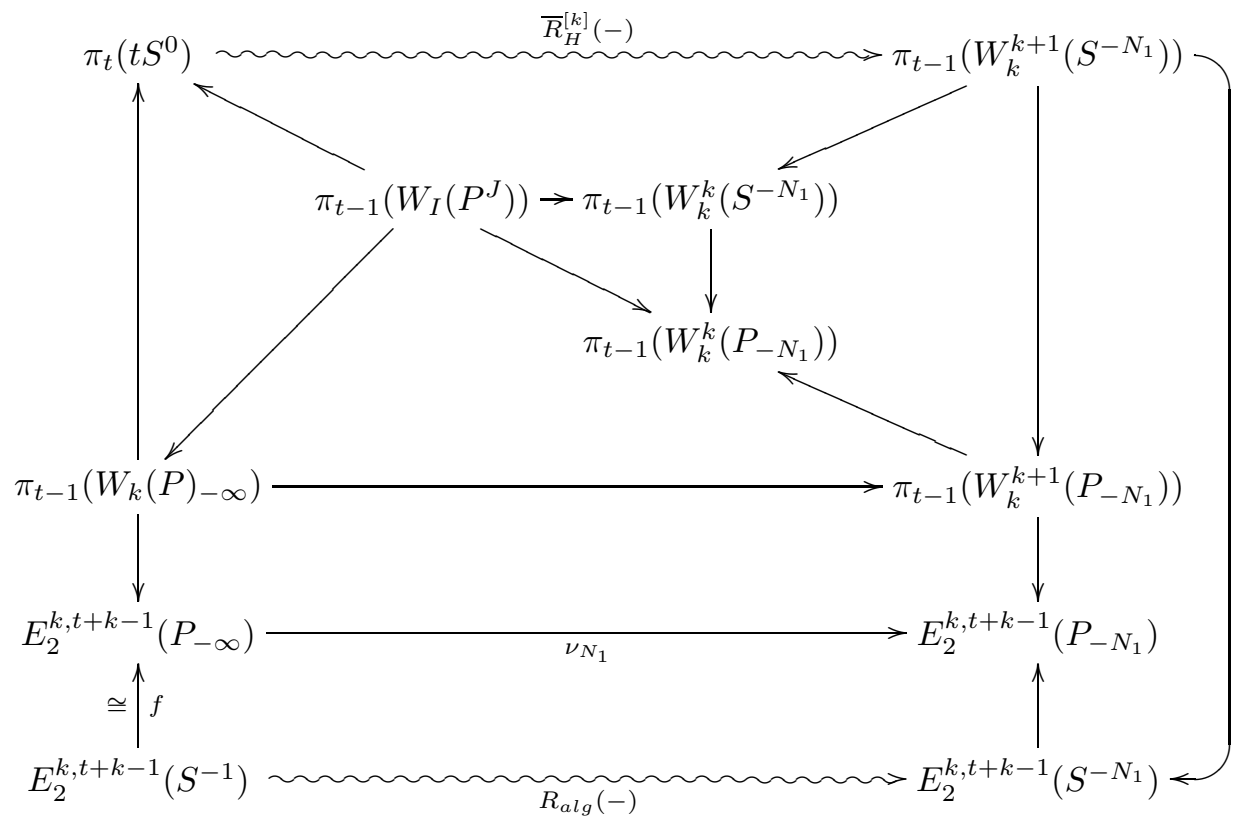

The element $\widetilde{\beta}$ maps to an element of $\bar{R}_{H}^{[k]}(\alpha)$ in $E_{2}^{k, t+k-1}\left(S^{-N_{1}}\right)$. The map $f$ is an isomorphism, so the image of $f_{1}^{\alpha}$ lifts to an element $\widetilde{\alpha} \in E_{2}^{k, t+k-1}\left(S^{-1}\right)$. This is the choice of $\widetilde{\alpha}$ which detects $\alpha$ that we appeal to in the statement of Theorem 5.3 . Recall that the image of $f_{1}^{\alpha}$ in $\pi_{t-1}\left(W_{k}^{k+1}\left(P_{-N_{1}}\right)\right)$ was $\gamma$, while the image of $\beta$ is $\widetilde{\gamma}$. Since the difference $\gamma-\widetilde{\gamma}$ maps to zero in $E_{2}^{k, t+k-1}\left(P_{-N_{1}}\right)$, we may conclude that the image of $\widetilde{\beta}$ in $E_{2}^{k, t+k-1}\left(S^{-N_{1}}\right)$ is also an element of the algebraic root invariant, provided the algebraic root invariant does not lie in a higher stem.

We have proven that either what is claimed in Theorem 5.10 holds, or the algebraic root invariant lives in a larger stem than $R_{H}^{[1]}(\alpha)$. We shall now show that this cannot happen. Let $M$ be maximal such that the image of $\widetilde{\alpha}$ in $E_{2}^{k, t+k-1}\left(S^{-1}\right)$ maps to zero under the composite

$$
E_{2}^{k, t+k-1}\left(S^{-1}\right) \stackrel{\nu_{M-1} \circ f}{\longrightarrow} E_{2}^{k, t+k-1}\left(P_{-M+1}\right) .
$$

By the algebraic Segal conjecture, such a finite $M$ exists. We wish to show that $N_{1}=M$. So far we know that $N_{1} \leq M$. In light of the definition of $N_{1}$, we simply must show that the $\alpha$ is sent to zero under the composition

$$
\pi_{t-1}\left(S^{-1}\right) \rightarrow \pi_{t-1}\left(W_{0}^{(k-1, k)}\left(P_{(-M+1, \infty)}\right)\right) .
$$


To this end consider the following diagram:

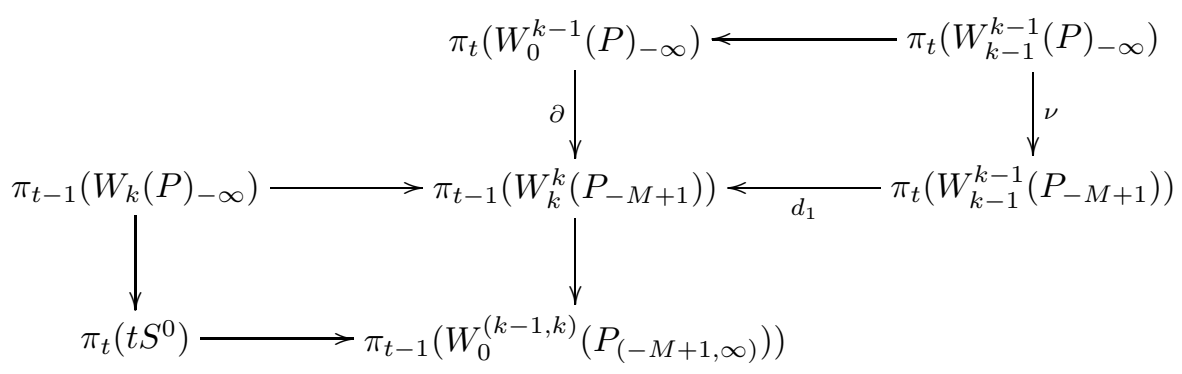

The central vertical column corresponds to part of the long exact sequence for a cofibration. The element $f_{1}^{\alpha} \in \pi_{t-1}\left(W_{k}(P)_{-\infty}\right)$ maps to the image of $\alpha$ in $\pi_{t}\left(t S^{0}\right)$. Let $g$ be the image of $f_{1}^{\alpha}$ in $\pi_{t-1}\left(W_{k}^{k}\left(P_{-M+1}\right)\right)$. By our choice of $M, g$ must vanish in $E_{2}$, that is to say, there must be an element $h \in \pi_{t}\left(W_{k-1}^{k-1}\left(P_{-M+1}\right)\right)$ such that $d_{1}(h)=g$. The map $\nu$ is surjective, because we are dealing with ordinary homology, and the map

$$
H_{*}(P)_{-\infty} \rightarrow H_{*}\left(P_{N}\right)
$$

is surjective for every $N$. Thus $h$ lifts to an element $\widetilde{h} \in \pi_{t}\left(W_{k-1}^{k-1}(P)_{-\infty}\right)$. Applying $\partial$ to the image of $\widetilde{h}$ in $\pi_{t}\left(W_{0}^{k-1}(P)_{-\infty}\right)$, we get $g$, so $g$ is in the image of $\partial$. Thus, by exactness of the vertical column, the image of $g$ in $\pi_{t-1}\left(W_{0}^{(k-1, k)}\left(P_{(-M+1, \infty)}\right)\right)$ is trivial. Thus the image of $\alpha$ in $\pi_{t}\left(t S^{0}\right)$ maps to zero in $\pi_{t-1}\left(W_{0}^{(k-1, k)}\left(P_{(-M+1, \infty)}\right)\right)$, which is what we were trying to prove.

\section{7. bo RESOLUTIONS}

The bo resolution for the sphere was the motivating example for the theorems in Section 5. The $d_{1}$ 's on the $v_{1}$-periodic summand of the 0- and 1-lines of the bo-resolution reflect the root invariants of the elements $2^{i}$ at the prime 2 . We do not rederive these root invariants, but satisfy ourselves in explaining how the theorems of Section 5 play out in this context. Alternatively, one might interpret this section as explaining how to use bo to compute the root invariants in the $K(1)$ local category. This section is furthermore meant to explain how the techniques of this paper, when applied to the small resolution of $L_{K(2)}\left(S^{0}\right)$ described in [13], could be used to compute root invariants in the $K(2)$-local category.

Recall that $\Sigma^{4} b s p$ is a summand of bo $\wedge \Sigma \overline{b o}$, hence its homotopy is a stable summand of the 1-line of the $E_{1}$-term of the bo-ASS. The composite of the $d_{1}$ of this spectral sequence with the projection onto the summand

$$
\pi_{t}(b o) \stackrel{d_{1}}{\longrightarrow} \pi_{t-1}(b o \wedge \overline{b o}) \rightarrow \pi_{t-1}\left(\Sigma^{4} b s p\right)
$$

is the map $\psi^{3}-1$ (up to a unit in $\mathbb{Z}_{(2)}$ ). The fiber of $\psi^{3}-1$ is the 2-primary $J$ spectrum. Figure 4 shows the $v_{1}$-periodic summand of the bo-ASS with differentials. In this figure, dots represent copies of $\mathbb{F}_{2}$ and circles represent copies of $\mathbb{Z}$. The $d_{1}$ differentials are (up to a 2-adic unit) multiplication by the power of 2 indicated. What survives are the $v_{1}$-periodic elements in $\pi_{*}^{S}$ at $p=2$.

Our theorems explain the relationship between $R_{b o}\left(2^{k}\right)$ and $R\left(2^{k}\right)$ for all $k>0$. It is quite straightforward to calculate $R_{b o}\left(2^{k}\right)$ (see [25]). Let $\eta, \alpha$, and $\beta$ be the 


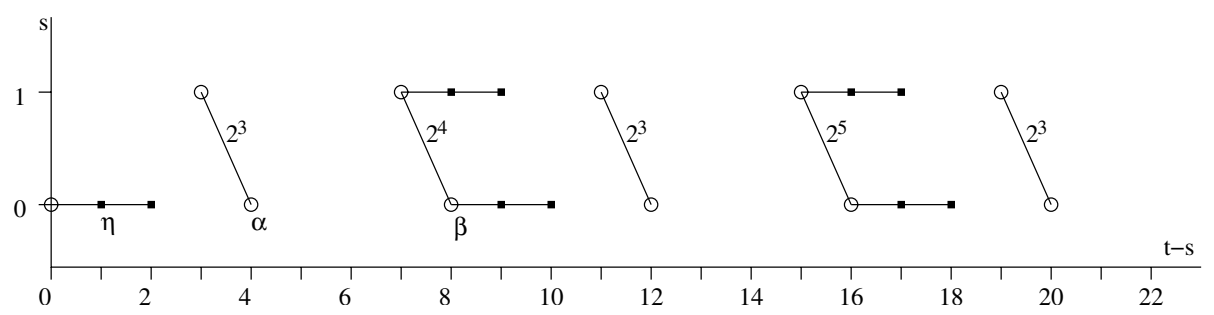

Figure 4 . The $v_{1}$-periodic summand of the bo-ASS

multiplicative generators of $\pi_{*}(b o)$ in dimensions 1,4 , and 8 , respectively. Then we have the following bo-root invariants:

$$
R_{b o}\left(2^{k}\right)= \begin{cases}\beta^{i}, & k=4 i, \\ \eta \beta^{i}, & k=4 i+1, \\ \eta^{2} \beta^{i}, & k=4 i+2, \\ \alpha \beta^{i}, & k=4 i+3 .\end{cases}
$$

These are the filtered root invariants $R_{b o}^{[0]}\left(2^{k}\right)$, by Proposition 5.7. For $k \equiv 1,2$ $(\bmod 4)$, the elements $R_{b o}^{[0]}\left(2^{k}\right)$ are in the Hurewicz image of bo, hence they are permanent cycles. They detect elements in $R\left(2^{k}\right)$.

For $k \equiv 3,4(\bmod 4)$, the elements $R_{b o}\left(2^{k}\right)$ are not in the bo-Hurewicz image. Therefore, the elements $R_{b o}^{[0]}\left(2^{k}\right)$ support differentials. We have

$$
d_{1} R_{b o}^{[0]}\left(2^{k}\right)=2 \cdot \widetilde{\alpha}_{k},
$$

where $\widetilde{\alpha}_{k}$ survives to a $v_{1}$-periodic element of order 2 in dimension $4 k-1$. In the bo-bifiltration of $2^{k}$, the $-N_{1}$ cell carries the zeroth filtered root invariant, where $N_{1}$ is given by

$$
N_{1}= \begin{cases}8 i+5, & k=4 i+3, \\ 8 i+1, & k=4 i .\end{cases}
$$

The first cell to attach to the $-N_{1}$ cell in $P_{-N_{1}}$ is the $-N_{1}+1$ cell, and the attaching map is the degree 2 map. Therefore, we may deduce from Theorem 5.3 that $\widetilde{\alpha}_{k}$ is an element of $R_{b o}^{[1]}\left(2^{k}\right)$. The element $\widetilde{\alpha}_{k}$ detects the homotopy root invariant $R\left(2^{k}\right)$.

\section{The algebraic Atiyah-Hirzebruch spectral sequence}

As discussed after the statement of Corollary 5.2 it is often the case that one may know that $R_{E}^{[k]}(\alpha)$ contains a permanent cycle in the $E$-Adams spectral sequence, but nothing else. One would want to conclude that this permanent cycle detects the homotopy root invariant $R(\alpha)$, but Theorem 5.1 says this is only true modulo obstructions in higher $E$-Adams filtration. However, it is sometimes the case that brute force computations in the $E_{2}$-term of the $E$-Adams spectral sequence for $P_{N}$ will yield enough data to eliminate such obstructions. This section is concerned with computations of the $E_{2}$-term $E_{2}\left(P_{N}\right)$. Specifically, we shall assume that $E=B P$ and describe the algebraic Atiyah-Hirzebruch spectral sequence (AAHSS), which computes $\operatorname{Ext}\left(B P_{*} P_{N}\right)$ from $\operatorname{Ext}\left(B P_{*}\right)$. In Section 9, we describe a procedure (Procedure 9.1) that explains how a limited knowledge of differentials in the AAHSS can be exploited to compute homotopy root invariants from filtered root invariants. 
The AAHSS is the spectral sequence obtained by applying Ext to the skeletal filtration of a complex. The AAHSS has appeared in various forms in the literature. In [22], Mahowald uses ordinary cohomology, and uses this spectral sequence to compute Ext of various stunted projective spaces. In [35], Sadofsky uses the $B P$ version that is the subject of this section. There is some overlap with this section and the computations in 35 .

We will first describe the AAHSS for computing the Adams-Novikov $E_{2}$-term $E_{2}\left(P_{N}\right)$, where $N \equiv-1(\bmod q)$. Let $F$ be the universal $p$-typical formal group law associated to $B P$, and write the $p$-series of $F$ as

$$
[p]_{F}(x)=\sum_{i \geq 0}^{F} v_{i} x^{p^{i}}=\sum_{j} c_{j} x^{(p-1) j+1},
$$

where $v_{i}$ are the Araki generators. Then we can say the following about the coefficients $c_{i}$ :

$$
\begin{aligned}
c_{0} & =p, \\
c_{1} & =v_{1}, \\
c_{j} & \equiv 0 \quad(\bmod p) \text { for } 1<j<p+1, \\
c_{p+1} & \equiv v_{2} \quad(\bmod p) .
\end{aligned}
$$

There are short exact sequences (compare with [35, 2.3])

$$
\begin{gathered}
0 \rightarrow B P_{*}\left(S^{k q-1}\right) \stackrel{\phi}{\rightarrow} B P_{*}\left(P_{N}^{k q-1}\right) \rightarrow B P_{*}\left(P_{N}^{k q}\right) \rightarrow 0, \\
0 \rightarrow B P_{*}\left(P_{N}^{(k-1) q}\right) \rightarrow B P_{*}\left(P_{N}^{k q-1}\right) \stackrel{\nu}{\rightarrow} B P_{*}\left(S^{k q-1}\right) \rightarrow 0,
\end{gathered}
$$

and these give rise to long exact sequences upon applying Ext. They have the following boundary homomorphisms:

$$
\begin{aligned}
& \delta_{1}: \operatorname{Ext}^{s, t}\left(B P_{*} P_{N}^{k q}\right) \rightarrow \operatorname{Ext}^{s+1, t}\left(B P_{*} S^{k q-1}\right), \\
& \delta_{2}: \operatorname{Ext}^{s, t}\left(B P_{*} S^{k q-1}\right) \rightarrow \operatorname{Ext}^{s+1, t}\left(B P_{*} P_{N}^{(k-1) q}\right) .
\end{aligned}
$$

For $l \leq k, B P_{*}\left(P_{l q-1}^{k q}\right)$ is generated as a $B P_{*}$-module by elements $e_{j q-1}$ in dimension $j q-1$. The map $\phi$ in the short exact sequence (8.1) is given by

$$
\phi\left(\iota_{k q-1}\right) \mapsto \sum_{i} c_{i} e_{q(k-i)-1}
$$

Splicing these sequences together creates an exact couple, and the resulting spectral sequence is the AAHSS. We shall index it just as one indexes the stable EHP spectral sequence [33, 1.5]. In fact, if $N=q-1$, then this is precisely the $B P$-algebraic stable EHP spectral sequence. Thus we have a spectral sequence

$$
E_{1}^{k, n, s} \Rightarrow \operatorname{Ext}^{s, s+k}\left(B P_{*} P_{N}\right),
$$

where the $E_{1}$ term is described as follows:

$$
\begin{aligned}
E_{1}^{k, 2 m, s} & =\mathrm{Ext}^{s, s+k}\left(B P_{*} S^{m q-1}\right), \\
E_{1}^{k, 2 m+1, s} & =\mathrm{Ext}^{s+1, s+k}\left(B P_{*} S^{m q-1}\right) .
\end{aligned}
$$

The indexing works out so that

$$
d_{r}: E_{r}^{k, n, s} \rightarrow E_{r}^{k-1, n-r, s+1} .
$$


If we wish to compute $\operatorname{Ext}\left(B P_{*} P_{N}\right)$ for $N=l q$, simply truncate the AAHSS for $\operatorname{Ext}\left(B P_{*} P_{N-1}\right)$ by setting $E_{1}^{k, 2 l, s}=0$ for all $k$ and $s$.

We shall refer to an element in the $E_{1}$-term of the AAHSS by its name in $\operatorname{Ext}\left(B P_{*}\right)$ and the cell that it is borne on. Thus if $\gamma \in \operatorname{Ext}\left(B P_{*}\right)$ is in $E_{1}^{k, 2 m, s}$, then we shall refer to it as $\gamma[m q-1]$. Likewise, if $\gamma$ is in $E_{1}^{k, 2 m+1, s}$, we shall refer to it as $\gamma[m q]$.

In order to implement Procedure 9.1 we shall need to know how to explicitly compute differentials in the filtered spectral sequence. It is useful to use the diagram below. In this diagram and the remainder of this section we will drop $B P_{*}$ from our Ext notation for compactness.

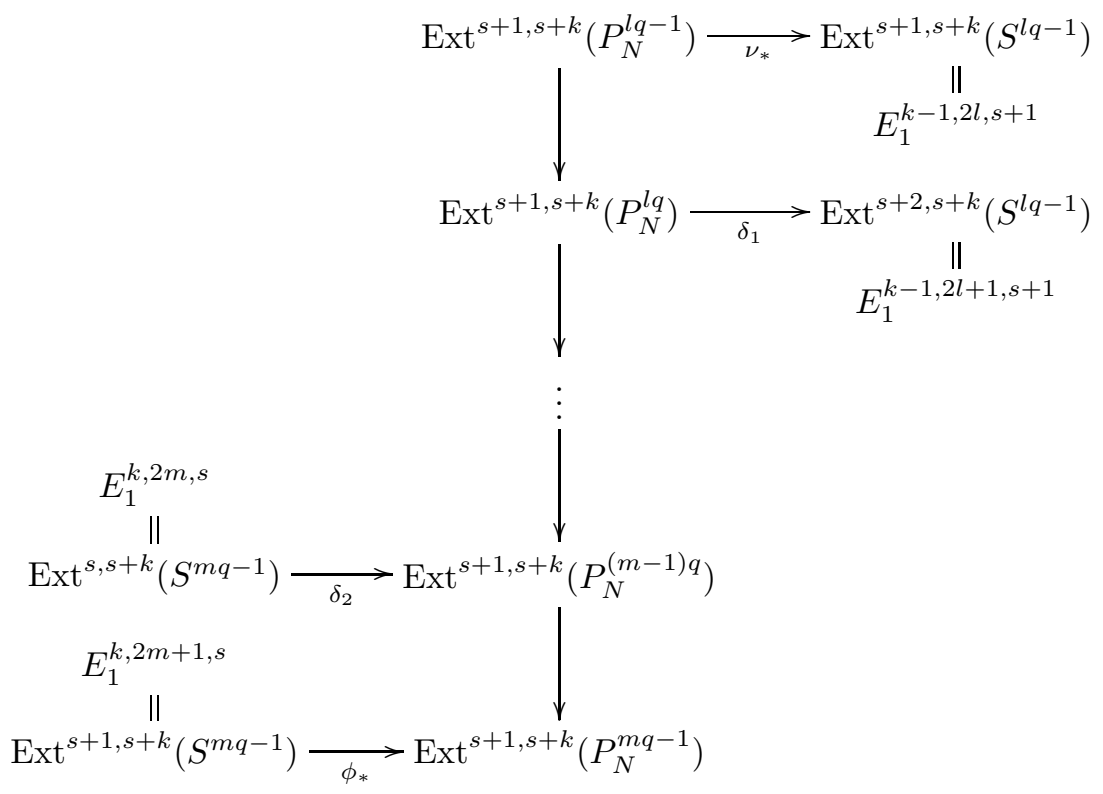

Suppose $\gamma[n]$ is an element of $E_{1}$, where $n=m q-\epsilon, \epsilon=0,1$. Let $\gamma_{1}$ be the image of $\gamma$ in $\operatorname{Ext}^{s+1, s+k}\left(P_{N}^{m q-1}\right)$ or $\operatorname{Ext}^{s+1, s+k}\left(P_{N}^{(m-1) q}\right)$, depending on the value of $\epsilon$. Then lift $\gamma_{1}$ as far as possible up the tower in the center of the above diagram. Suppose that $\gamma_{1}$ lifts to $\gamma_{2} \in \mathrm{Ext}^{s+1, s+k}\left(P_{N}^{l q-\epsilon_{1}}\right)$, where $\epsilon_{1}=0,1$. Then if $\gamma_{3}$ is the image of $\gamma_{2}$ under $\nu_{*}$ or $\delta_{1}$ (depending on the value of $\epsilon_{1}$ ), there is an AAHSS differential

$$
d_{r}(\gamma[n])=\gamma_{3}\left[n^{\prime}\right]
$$

where $n^{\prime}=l q-\epsilon$.

The above description makes the following proposition clear. In the statement of this proposition, and in what follows, when we say that differentials are computed modulo $I_{n}$, we mean is that we are considering their images in $\operatorname{Ext}\left(B P_{*}(-) / I_{n}\right)$.

Proposition 8.1. Modulo $I_{n}$, we have the differential

$$
d_{2 p^{n}-1}(\alpha[m q])=v_{n} \cdot \alpha\left[m q-2 p^{n}+1\right] .
$$

Proof. Take $\alpha \in \mathrm{Ext}^{s+1, s+k}\left(S^{m q-1}\right)$. Clearly, it is the case that

$$
\phi_{*}(\alpha) \underset{\left(\bmod I_{n}\right)}{\equiv} v_{n} \alpha\left[m q-2 p^{n}+1\right]+\cdots
$$


lifts to $\operatorname{Ext}^{s+1, s+k}\left(P^{m q-2 p^{n}+1}\right)$, and the image of this lift under the map $\nu_{*}$ is

$$
v_{n} \cdot \alpha \in \mathrm{Ext}^{s+1, s+k}\left(S^{m q-2 p^{n}+1}\right) .
$$

In order to compute $\delta_{1}$ and $\delta_{2}$, we must know something of the $B P_{*} B P$ comodule structure of $B P_{*}\left(P_{N}\right)$. It suffices to understand the $B P_{*} B P$ comodule structure of $B P_{*}(B \mathbb{Z} / p)$, since $P_{0}$ is a stable summand of $B \mathbb{Z} / p_{+}$. The $B P_{*} B P$ comodule structure of $B P_{*}\left(P_{N}\right)$ may then be deduced from James periodicity. In [35], it is proven that there is a cofiber sequence

$$
\mathbb{C} P^{\infty} \rightarrow\left(\mathbb{C} P^{\infty}\right)^{\xi^{p}} \rightarrow \Sigma B \mathbb{Z} / p
$$

(where $\xi$ is the canonical complex line bundle on $\mathbb{C} P^{\infty}$ ) which induces the short exact sequence

$$
0 \rightarrow B P_{*} \mathbb{C} P^{\infty} \rightarrow B P_{*}\left(\mathbb{C} P^{\infty}\right)^{\xi^{p}} \rightarrow B P_{*} \Sigma B \mathbb{Z} / p \rightarrow 0 .
$$

Since $B P_{*}\left(\mathbb{C} P^{\infty}\right)^{\xi^{p}}$ surjects onto $B P_{*} \Sigma B \mathbb{Z} / p$, it suffices to understand the $B P_{*} B P$ comodule structure of the former.

Recall that $B P^{*}\left(\mathbb{C} P^{\infty}\right)=B P^{*}[[x]]$, where $x$ is the Euler class of $\xi$. Therefore, the image of the inclusion

$$
B P^{*}\left(\mathbb{C} P^{\infty}\right)^{\xi^{p}} \rightarrow B P^{*}\left(\mathbb{C} P^{\infty}\right)
$$

is the ideal

$$
[p]_{F}(x) \cdot B P^{*}[[x]] \subseteq B P^{*}\left(\mathbb{C} P^{\infty}\right) .
$$

We shall identify $B P^{*}\left(\mathbb{C} P^{\infty}\right)^{\xi^{p}}$ with this ideal.

Let $y_{2 k}$ be the generator in $B P_{2 k}\left(\left(\mathbb{C} P^{\infty}\right)^{\xi^{p}}\right)$ dual to $[p]_{F}(x) \cdot x^{k-1}$. Let $f(x)$ be the formal power series over $B P_{*} B P$ whose inverse is given by

$$
f^{-1}(x)=\sum_{i \geq o}^{F} t_{i} x^{p^{i}}
$$

The power series $f(x)$ is the universal strict isomorphism of $p$-typical formal group laws.

Proposition 8.2. The $B P_{*} B P$ coaction on $y_{2 k}$ is given by

$$
\psi\left(y_{2 k}\right)=\sum_{i+j=k}\left(\frac{f\left([p]_{F}(x)\right)}{[p]_{F}(x)}(f(x))^{j-1}\right)_{k-1} \otimes y_{2 j} .
$$

Here the subscript $k-1$ indicates the coefficient of $x^{k-1}$.

Proof. We digress for a moment on cooperations. For a spectrum $X$, the left unit $\eta_{L}: B P \rightarrow B P \wedge B P$ induces the $B P_{*} B P$ coaction

$$
\psi: B P_{*}(X) \stackrel{\left(\eta_{L}\right)_{*}}{\longrightarrow}(B P \wedge B P)_{*}(X) \cong B P_{*} B P \otimes_{B P_{*}} B P_{*}(X) .
$$

It is sometimes convenient to consider the dual coaction on cohomology

$$
\psi^{*}: B P^{*}(X) \stackrel{\left(\eta_{L}\right)_{*}}{\longrightarrow}(B P \wedge B P)^{*}(X) .
$$

In Theorem 11.3 of Part II of [1], Adams describes the $M U_{*} M U$ coaction on $M U_{*}\left(\mathbb{C} P^{\infty}\right)$. When translated into $B P$-theory, the coaction formula reads

$$
\psi\left(e_{2 k}\right)=\sum_{i+j=k}\left((f(x))^{j}\right)_{k} \otimes e_{2 j} .
$$


Here, $e_{2 k}$ is the generator of $B P_{2 k}\left(\mathbb{C} P^{\infty}\right)$ dual to $x^{k} \in B P^{2 k}\left(\mathbb{C} P^{\infty}\right)$. Upon dualization, we get the following formula for the dual coaction on an element $h(x)$ in $B P^{*}\left(\mathbb{C} P^{\infty}\right)$ :

$$
\psi^{*}(h(x))=f_{*} h(f(x)) .
$$

The polynomial $f_{*} h(x)$ is the polynomial obtained from applying the right unit to the coefficients of $h(x)$. We deduce the dual coaction on an element $[p]_{F}(x) \cdot h(x)$ in $B P^{*}\left(\left(\mathbb{C} P^{\infty}\right)^{\xi^{p}}\right)$, regarded as the ideal $\left([p]_{F}(x)\right)$ contained in $B P^{*}[[x]]$.

$$
\begin{aligned}
\psi^{*}\left([p]_{F}(x) \cdot h(x)\right) & =f_{*}[p]_{F}(f(x)) \cdot f_{*} h(f(x)) \\
& =[p]_{f_{*} F}(f(x)) \cdot f_{*} h(f(x)) \\
& =f\left([p]_{F}(x)\right) \cdot f_{*} h(f(x)) \\
& =[p]_{F}(x) \cdot \frac{f\left([p]_{F}(x)\right)}{[p]_{F}(x)} \cdot f_{*} h(f(x)) .
\end{aligned}
$$

Here, $f_{*} F$ is the pushforward of the formal group law $F$ under the map $f$. Letting $h(x)=x^{j-1}$ and dualizing, we have the desired formula for the coaction on $B P^{*}\left(\left(\mathbb{C} P^{\infty}\right)^{\xi^{p}}\right)$.

We wish to use the above coaction formula to compute differentials in the AAHSS with $p \geq 3$. The first few terms of the relevant power series are listed below. Everything in what follows is written in terms of Hazewinkel generators:

$$
\begin{gathered}
{[p]_{F}(x)=p x+\left(1-p^{p-1}\right) v_{1} x^{p}+\mathcal{O}\left(x^{p}+1\right),} \\
f(x)=x-t_{1} x^{p}+\left(p t_{1}^{2}+v_{1} t_{1}\right) x^{2 p-1}+\mathcal{O}\left(x^{2 p}\right), \\
\frac{f\left([p]_{F}(x)\right)}{[p]_{F}(x)}= \\
\quad 1-p^{p-1} t_{1} x^{p-1} \\
+p^{p-2}\left(p^{p+1} t_{1}^{2}+\left(1-p-p^{p-1}+2 p\right) v_{1} t_{1}\right) x^{2 p-2}+\mathcal{O}\left(x^{2 p-1}\right) .
\end{gathered}
$$

We may compute these series further if we work modulo the ideal $I_{2}=\left(p, v_{1}\right)$ :

$$
\begin{gathered}
{[p]_{F}(x) \equiv v_{2} x^{p^{2}}+\mathcal{O}\left(x^{p^{2}}+1\right) \quad\left(\bmod I_{2}\right),} \\
f(x) \equiv x-t_{1} x^{p}+\mathcal{O}\left(x^{p^{2}}\right) \quad\left(\bmod I_{2}\right), \\
\frac{f\left([p]_{F}(x)\right)}{[p]_{F}(x)} \equiv 1+\mathcal{O}\left(x^{p^{2}-1}\right) \quad\left(\bmod I_{2}\right) .
\end{gathered}
$$

These formulas give rise to the following proposition. 
Proposition 8.3. In the filtered spectral sequence, the following formulas for differentials hold up to multiplication by a unit in $\mathbb{Z}_{(p)}$ :

$$
\begin{aligned}
& d_{2}(\alpha[k q-1]) \doteq\left\{\begin{array}{lll}
\alpha_{1} \cdot \alpha[(k-1) q-1], & k \neq 0 & (\bmod p), \\
0, & k \equiv 0 & (\bmod p),
\end{array}\right. \\
& d_{2}(\alpha[k q]) \underset{\left(\bmod I_{1}\right)}{\doteq}\left\{\begin{array}{lll}
\alpha_{1} \cdot \alpha[(k-1) q], & k \neq 1 & (\bmod p), \\
0, & k \equiv 1 & (\bmod p),
\end{array}\right. \\
& d_{q}(\alpha[k q-1]) \underset{\left(\bmod I_{2}\right)}{\dot{\epsilon}}\left\{\begin{array}{lll}
\langle\alpha, \underbrace{\alpha_{1}, \ldots, \alpha_{1}}_{p-1}\rangle[(k-p+1) q-1], & k \equiv-1 & (\bmod p), \\
0, & k \neq-1 & (\bmod p),
\end{array}\right. \\
& d_{q}(\alpha[k q]) \underset{\left(\bmod I_{2}\right)}{\dot{\epsilon}}\left\{\begin{array}{lll}
\langle\alpha, \underbrace{\alpha_{1}, \ldots, \alpha_{1}}_{p-1}\rangle[(k-p+1) q], & k \equiv 0 & (\bmod p), \\
0, & k \neq 0 & (\bmod p) .
\end{array}\right.
\end{aligned}
$$

Proof. We shall begin by computing $d_{2}(\alpha[k q-1])$. In light of the coaction equation 8.3. we compute from (8.5) and (8.6)

$$
\frac{f\left([p]_{F}(x)\right)}{[p]_{F}(x)}(f(x))^{r}=x^{r}-\left(r+p^{p-1}\right) t_{1} x^{r+p-1}+\mathcal{O}\left(x^{r+p}\right) .
$$

We may conclude that the coaction formula on the generator $e_{k q-1}$ of $B P_{*}\left(P_{0}^{\infty}\right)$ is given by

$$
\psi\left(e_{k q-1}\right)=1 \otimes e_{k q-1}+((k-1)(p-1)-1) t_{1} \otimes e_{(k-1) q-1}+\cdots
$$

Now $t_{1}$ represents $\alpha_{1}$ in the cobar complex, and $p \cdot \alpha_{1}=0$.

We now compute $d_{2}$ :

$$
\delta_{2}(\alpha[k q-1])=((k-1)(p-1)-1) \alpha \mid t_{1}[(k-1) q-1]+\cdots
$$

and it follows immediately that

$$
\begin{aligned}
d_{2}(\alpha[k q-1]) & =((k-1)(p-1)-1) \alpha_{1} \cdot \alpha[(k-1) q-1] \\
& \equiv-k \alpha_{1} \cdot \alpha[(k-1) q-1] \quad(\bmod p) .
\end{aligned}
$$

Of course, since $p \cdot \alpha_{1}=0$, one needs only to compute this differential modulo $p$.

Now we shall deal with $d_{2}(\alpha[k q])$. We shall work modulo $I_{1}$.

$$
\phi_{*} \alpha[k q] \equiv v_{1} \cdot \alpha v_{1}[(k-1) q-1]+\cdots .
$$

Therefore $\phi_{*} \alpha[k q]$ lifts to $\operatorname{Ext}\left(P_{0}^{(k-1) q}\right)$. So

$$
d_{2}(\alpha[k q])=\delta_{1}\left(v_{1} \cdot \alpha[(k-1) q-1]+\cdots\right)
$$


The boundary homomorphism $\delta_{1}$ is computed below on the level of cobar complexes:
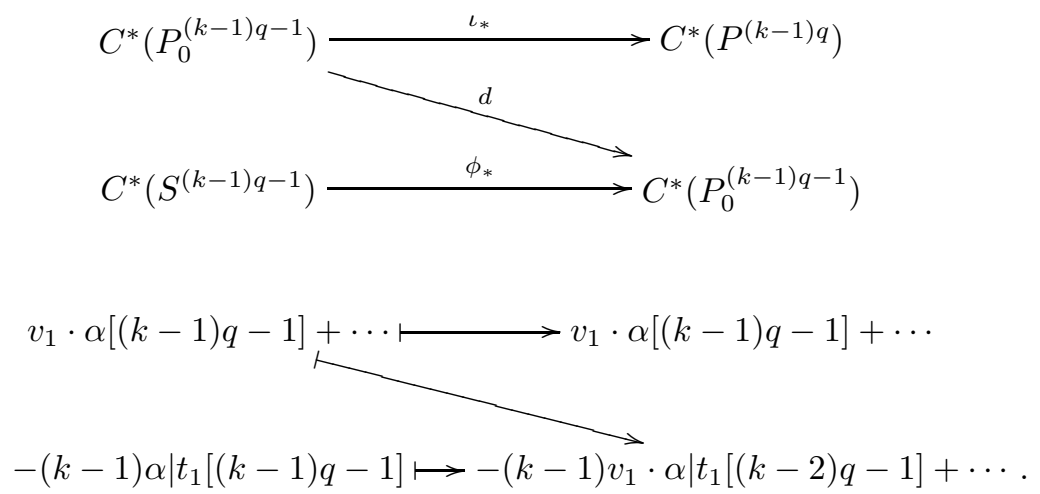

Here, $C^{*}(-)$ represents the cobar complex. We conclude that

$$
\delta_{1}\left(v_{1} \cdot \alpha[(k-1) q-1]+\cdots\right) \equiv(1-k) \alpha \mid t_{1}[(k-1) q-1] \quad\left(\bmod I_{1}\right) .
$$

The $d_{q}$ 's are proved similarly. The relevant information to be gathered from Equations (8.8) and (8.9) is

$$
\begin{aligned}
\frac{f\left([p]_{F}(x)\right)}{[p]_{F}(x)}(f(x))^{r} \underset{\left(\bmod I_{2}\right)}{\equiv} x^{r} & +\left(\begin{array}{c}
r \\
1
\end{array}\right)\left(-t_{1}\right) x^{r+(p-1)}+\cdots \\
& +\left(\begin{array}{c}
r \\
p-1
\end{array}\right)\left(-t_{1}\right)^{p-1} x^{r+(p-1)^{2}}+\mathcal{O}\left(x^{r+(p-1)^{2}+1}\right) .
\end{aligned}
$$

The Massey product

$$
\langle\alpha, \underbrace{\alpha_{1}, \ldots, \alpha_{1}}_{p-1}\rangle
$$

corresponds (up to multiplication by a unit) to

$$
\alpha \mid t_{1}^{p-1}+\cdots
$$

in the cobar complex. We have that

$$
d_{q}(\alpha[k q-1]) \underset{\left(\bmod I_{2}\right)}{\in}\left(\begin{array}{c}
r \\
p-1
\end{array}\right)\langle\alpha, \underbrace{\alpha_{1}, \ldots, \alpha_{1}}_{p-1}\rangle[(k-p+1) q-1],
$$

where $r=(k-p+1)(p-1)-1$. Consider the coefficient

$$
\left(\begin{array}{c}
r \\
p-1
\end{array}\right)=\frac{r \cdot(r-1) \cdots(r-p+2)}{(p-1) !} .
$$

Since $p \nmid p-1$, in order for $p$ to not divide this binomial coefficient, it must be the case that $r \equiv-1(\bmod p)$. In terms of $k$, this translates to $k \equiv-1(\bmod p)$.

Just as in the calculation of $d_{2} \alpha[k q]$, the map $\delta_{1}$ introduces an offset in coefficients which results in the claimed formula for $d_{q} \alpha[k q]$.

It is interesting to note that these differentials, combined with the image of $J$ differentials, are enough to compute the AAHSS completely through a certain range at odd primes.

In $\operatorname{Ext}\left(B P_{*}\right)$, many elements live in an $\alpha_{1}-\beta_{1}$ tower, that is, a copy of $P\left[\beta_{1}\right] \otimes$ $E\left(\alpha_{1}\right)$. The following general observation says that elements of the $E_{1}$-term of the 
AAHSS which lie within an $\alpha_{1}-\beta_{1}$ tower typically either support differentials or are the target of differentials.

Proposition 8.4. Let $\gamma \in \operatorname{Ext}\left(B P_{*}\right)$. Suppose furthermore that there exist elements $\lambda$ and $\mu$ in the $E_{2}$ term of the Adams-Novikov spectral sequence such that either

$$
\begin{gathered}
\alpha_{1} \lambda=\gamma, \\
\langle\gamma, \underbrace{\alpha_{1}, \ldots, \alpha_{1}}_{p-1}\rangle=\mu
\end{gathered}
$$

or

$$
\begin{gathered}
\langle\lambda, \underbrace{\alpha_{1}, \ldots, \alpha_{1}}_{p-1}\rangle=\gamma, \\
\alpha_{1} \gamma=\mu .
\end{gathered}
$$

(Here we insist that the Massey products have no indeterminacy.) Assume furthermore that $p$ and $v_{1}$ do not divide any of $\lambda, \mu$, or $\gamma$. Then, in the AAHSS, $\gamma[n]$ is either killed by a differential, or supports a non-trivial differential.

Proof. The condition on $p$ and $v_{1}$ not dividing any of the elements $\lambda, \mu$, or $\gamma$ is just to ensure that we may use the formulas in Proposition 8.3. Let us suppose that $\gamma$ satisfies the first set of conditions.

Case $1: n \equiv-1(\bmod q):$ Write $n=m q-1$.

Subcase (a): $m \equiv-1(\bmod p)$ : Then $d_{q} \gamma[m q-1]=\mu[(m-p+1) q-1]$ or $d_{i} \gamma[m q-1] \neq 0$ for some $i<q$.

Subcase $(\mathrm{b}): m \not \equiv-1(\bmod p)$ : Then $d_{2} \lambda[(m+1) q-1]=\gamma[m q-1]$.

Case 2: $n \equiv 0(\bmod q)$ : Write $n=m q$.

Subcase $(\mathrm{a}): m \equiv 0(\bmod p)$ : Then either $d_{q} \gamma[m q]=\mu[(m-p+1) q]$ or we have $d_{i} \gamma[m q] \neq 0$ for some $i<q$.

Subcase $(\mathrm{b}): m \not \equiv 0(\bmod p)$ : Then $d_{2} \lambda[(m+1) q]=\gamma[m q]$.

Suppose now that $\gamma$ satisfies the second set of conditions.

Case 1: $n \equiv-1(\bmod q):$ Write $n=m q-1$.

Subcase $(\mathrm{a}): m \equiv 0(\bmod p)$ : Then $d_{q} \lambda[(m+p-1) q-1]=\gamma[m q-1]$.

Subcase $(\mathrm{b}): m \not \equiv 0(\bmod p)$ : Then $d_{2} \gamma[m q-1]=\mu[(m-1) q-1]$.

Case 2: $n \equiv 0(\bmod q)$ : Write $n=m q$.

Subcase $(\mathrm{a}): m \equiv 1(\bmod p)$ : Then $d_{q} \lambda[(m+p-1) q]=\gamma[m q]$.

Subcase (b): $m \neq \equiv 1(\bmod p)$ : Then $d_{2} \gamma[m q]=\mu[(m-1) q]$ or $d_{1} \gamma[m q] \neq$ 0 . (This is the case of $p \cdot \gamma \neq 0$.)

It is also useful to record all of the differentials supported by the $v_{1}$-periodic elements of $\operatorname{Ext}\left(B P_{*}\right)$ for $p>2$ in the AAHSS. These results are used in executing Step 4 of Procedure 9.1 .

Proposition 8.5. In the AAHSS, the differentials supported by ImJ elements whose targets are ImJ elements are given by

$$
\begin{aligned}
d_{1}(1[k q]) & =p[k q-1], \\
d_{1}\left(\alpha_{i / j}[k q]\right) & =\alpha_{i /(j-1)}[k q-1] \text { for } j>1, \\
d_{2 j}(1[k q-1]) & \doteq \tilde{\alpha}_{j}[(k-j) q-1], \text { where } \nu_{p}(k)=j-1, \\
d_{2 j+1}\left(\alpha_{i}[k q]\right) & \doteq \tilde{\alpha}_{i+j}[(k-j) q-1], \text { where } \nu_{p}(k+i)=j-1 .
\end{aligned}
$$


(Here $\tilde{\alpha}_{l}$ is $\alpha_{l / m}$ for $m=\nu_{p}(l)+1$. It is the additive generator of $i m J$ in the $l q-1$ stem.)

Proof. These differentials detect the corresponding differentials in the AHSS which converge to the stable homotopy of $P_{N}$. These differentials were computed for $P_{0}$ by Thompson in 38 and are summarized in 33 .

There are still $v_{1}$-periodic elements of $\operatorname{Ext}\left(B P_{*}\right)$ which are eligible to support differentials in the filtered spectral sequence (or subsequently in the Adams-Novikov spectral sequence for $P_{N}$ ) whose targets are elements which are not $v_{1}$-periodic. These elements are described below.

Corollary 8.6. The only elements in imJ in the AAHSS for $P_{-\infty}$ which are neither targets nor sources of the differentials described in Proposition 8.5 are

$$
\tilde{\alpha}_{i}[k q-1],
$$

where $p^{i} \mid(k+i)$.

We obtain the following consequence which will become quite relevant when we execute Step 4 of Procedure 9.1.

Proposition 8.7. None of the elements $\beta_{i / j}[k q]$ are the target of differentials in the AAHSS or subsequently in the Adams-Novikov spectral sequence for $\pi_{*}\left(P_{N}\right)$.

Proof. The only elements which could kill $\beta_{i / j}[k q]$ in the filtered spectral sequence are of the form $\alpha_{n}[m q]$ and $1[m q-1]$. But these cannot, by Corollary 8.6. There are no elements in the $E_{2}$ term of the Adams-Novikov spectral sequence which can kill $\beta_{i / j}[k q]$. Such elements would have to lie in Adams-Novikov filtration 0.

\section{Procedure For low-Dimensional Calculations of root invariants}

In this section, we let $E=B P$, and concentrate on $B P$-filtered root invariants. The following procedure is used in later sections to compute homotopy root invariants from filtered root invariants using Theorem 5.1. It only has a chance to work through a finite range, and is very crude. We state it mainly to codify everything that must be checked in general to see that a filtered root invariant detects a homotopy root invariant.

Procedure 9.1. Suppose we are in the situation where we know $\beta$ is an element of $R_{B P}^{[k]}(\alpha)$, and we know that $\beta$ is a permanent cycle. Let $\iota_{-N}$ be the inclusion of the $-N$ cell of $P_{-N}$, where $-N=-N_{i}$ is the index of the bifiltration of $\alpha$ that corresponds to the cell that bears the $k^{\text {th }}$ filtered root invariant. Let $\nu_{-N} \alpha$ be the image of $\alpha$ in $\pi_{*}\left(P_{-N}\right)$. Then Theorem 5.1 tells us that $\nu_{-N} \alpha=\iota_{-N} \beta$ modulo elements in higher Adams-Novikov filtration.

Step 1: Make a list

$$
\gamma_{i}\left[-n_{i}\right]
$$

of additive generators in the $E_{1}$ term of the AAHSS for $\operatorname{Ext}\left(P_{-N}\right)$, where $\gamma_{i}$ lies in Adams-Novikov filtration $s_{i}$ and stem $k_{i}$, satisfying the following:

(1) The homological degree $s_{i}$ satisfies

$$
s_{i}>\left\{\begin{array}{lll}
k & n_{i} \equiv 1 \quad(\bmod q), \\
k+1 & n_{i} \equiv 0 \quad(\bmod q) .
\end{array}\right.
$$

(2) The stem $k_{i}$ is greater than $p \cdot|\alpha|$, and less than $|\beta|$. 
(3) We have $n_{i}=k_{i}-|\alpha|+1$, and $n_{i} \equiv 0,1(\bmod q)$.

These are precisely the conditions required for $\gamma_{i}\left[n_{i}\right]$ to be a candidate to survive in the AAHSS to an element in the same stem, but higher AdamsNovikov filtration, as $\iota_{-N} \beta$. Condition (3) is a consequence of $P_{-N}$ having cells in dimensions congruent to $0,-1$ modulo $q$. We apply the first inequality in (2) because Jones's theorem [29] will preclude the possibility of these surviving to detect the difference of $\iota_{-N} \bar{\beta}$ and $\nu_{-N} \alpha$. Thus we have

$\nu_{-N} \alpha=\beta[-N]+\sum_{i} a_{i} \gamma_{i}\left[-n_{i}\right]+($ terms born on cells $<-(p-1)|\alpha|+1)$.

Step 2: Attempt to determine which $\gamma_{i}\left[n_{i}\right]$ are killed in the filtration spectral sequence, or subsequently, in the Adams-Novikov spectral sequence for computing $\pi_{*}\left(P_{-N}\right)$. This will limit the possibilities for what can detect the difference of $\iota_{-N} \bar{\beta}$ and $\nu_{-N} \alpha$.

Step 3: First eliminate those $\gamma_{i}$ which are not permanent cycles in the ANSS. These cannot be root invariants. Then, attempt to show that every remaining non-trivial linear combination of the elements $\gamma_{i}\left[n_{i}\right]$ which are not killed in the AAHSS actually supports a non-trivial differential in the AHSS for computing $\pi_{*}\left(P_{-\infty}\right)$. Suppose this is the case. Then, if $\iota_{-N} \bar{\beta}-\nu_{-N} \alpha$ is nontrivial and is born on cells above the $-N$-cell, it will project non-trivially to $\pi_{*}\left(P_{-M}\right)$ for $M<N$, and will represent the image of the homotopy root invariant. The homotopy root invariant is a permanent cycle in the AHSS for $\pi_{*}\left(P_{-\infty}\right)$. Thus we may assume that the difference $\iota_{-N} \bar{\beta}-\nu_{-N} \alpha$ is actually born on cells above the $-(p-1)|\alpha|+1$ cell. If this difference was non-trivial, we would violate Jones's theorem 29. The difference is therefore trivial, and $\iota_{-N} \bar{\beta}=\nu_{-N} \alpha$.

Step 4: We will have shown that $\bar{\beta} \in R(\alpha)$ if we know that $\iota_{-N} \bar{\beta} \neq 0$. It would suffice to show that $\beta[-N]$ is not killed in the AAHSS, and also survives to a non-zero element in the ANSS for computing $\pi_{*}\left(P_{-N}\right)$. Form a list of all of the elements

$$
\eta_{i}\left[n_{i}\right]
$$

in the $E_{1}$-term of the AAHSS, where $\eta_{i}$ is in Adams-Novikov filtration $s_{i}$ and stem $k_{i}$ such that:

$$
\begin{aligned}
& \text { (1) } s_{i} \leq \begin{cases}k & -N \equiv-1(\bmod q), \\
k-1 & -N \equiv 0(\bmod q),\end{cases} \\
& \text { (2) } k_{i} \leq|\beta|, \\
& \text { (3) } n_{i}=-N+|\alpha|-k_{i}+1 \equiv 0,-1(\bmod q) .
\end{aligned}
$$

These are precisely the elements which have a chance of killing $\beta[-N]$ in the AAHSS, or subsequently in the ANSS. Next, show that these elements actually do not kill $\beta[-N]$.

Remark 9.2. By comparing the definition of differentials in the AAHSS with the differentials in the AHSS, it is clear that if $\gamma$ detects $\bar{\gamma}$ in the ANSS for computing $\pi_{*}^{S}$, then if $\gamma[n]$ supports a non-trivial AAHSS differential

$$
d_{r}(\gamma[n])=\eta[m]
$$


where $\eta$ is a permanent cycle in the ANSS, then either $\bar{\gamma}$ supports a non-trivial AHSS $d_{i}$ for $i<r$, or there is an AHSS differential

$$
d_{r}(\bar{\gamma}[n])=\bar{\eta}[m]
$$

where $\eta$ detects $\bar{\eta}$. However, the differential given in Equation (9.1) may be trivial in the AHSS if $\bar{\eta}$ is the target of a shorter differential.

Since in Step 3 of Procedure 9.1 we are only concerned with whether the elements $\bar{\gamma}_{i}\left[n_{i}\right]$ support non-trivial differentials in the AHSS, it suffices to show that the elements $\gamma_{i}\left[n_{i}\right]$ support non-trivial differentials in the AAHSS. One must then make sure that the targets of the AAHSS differentials are not the targets of shorter differentials in the AHSS.

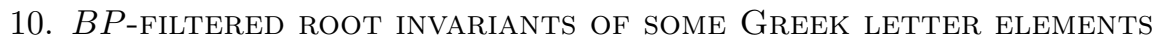

In this section we compute the first two $B P$-filtered root invariants of some chromatic families. If $\alpha=\alpha_{i / j_{1}, \ldots, j_{k}}^{(n)}$ is the $n^{\text {th }}$ algebraic Greek letter element of the ANSS which survives to a non-trivial element of $\pi_{*}^{S}$, then one might expect that it should be the case that

$$
\alpha_{i / j_{1}, \ldots, j_{k}}^{(n+1)} \dot{\in} R_{B P}^{[n+1]}\left(\alpha_{i / j_{1}, \ldots, j_{k}}^{(n)}\right) .
$$

The purpose of this section is to show that Equation (10.1) holds for $n=0$ at all primes and for $n=1$ at odd primes. Modulo an indeterminacy group which we do not compute, we also show that the $n=1$ case of Equation (10.1) is true at the prime 2. Throughout this section, for a ring spectrum $E$, we shall let $\widetilde{E} \simeq \Sigma \bar{E}$ denote the cofiber of the unit. Also, whereas in Section 8 we used Hazewinkel generators, in this section we always use Araki generators. This is because the $p$-series is more naturally expressed in the Araki generators.

For appropriate $i$ and $j$, the elements which generate the 1-line of the ANSS are $\alpha_{i / j} \in B P_{*} B P$ and are given by

$$
\alpha_{i / j}=\frac{\eta_{R}\left(v_{1}\right)^{i}-v_{1}^{i}}{p^{j}}
$$

Proposition 10.1. The first two filtered root invariants of $p^{i}$ are given by

$$
\begin{aligned}
\left(-v_{1}\right)^{i} & \in R_{B P}^{[0]}\left(p^{i}\right), \\
(-1)^{i} \cdot \alpha_{i} & \in R_{B P}^{[1]}\left(p^{i}\right) .
\end{aligned}
$$

Define, for appropriate $i$ and $j$,

$$
\widetilde{\beta}_{i / j}=\frac{\left(v_{2}+v_{1} t_{1}^{p}-v_{1}^{p} t_{1}\right)^{i}-v_{2}^{i}}{v_{1}^{j}} \in B P_{*} B P .
$$

Observe that the image of $\widetilde{\beta}_{i / j}$ in $B P_{*} B P / I_{1}$ coincides with

$$
\frac{\eta_{R}\left(v_{2}\right)^{i}-v_{2}^{i}}{v_{1}^{j}}
$$

It follows from the definition of the algebraic Greek letter elements that, in the cobar complex, $d_{1}\left(\widetilde{\beta}_{i / j}\right)=p \cdot \beta_{i / j}$ unless $p=2$ and $i=j=1$, in which case $\widetilde{\beta}_{1}=\alpha_{2 / 2}$, which is a permanent cycle that detects $\nu$. 
In order to compute the first filtered root invariants of the elements $\alpha_{i / j}$, we first compute the $B P \wedge \widetilde{B P}$-root invariants, and then invoke Corollary [5.9, Note that $\widetilde{B P}=\Sigma \overline{B P}$, so the $B P \wedge \widetilde{B P}$-root invariant is just the suspension of the $B P \wedge \overline{B P}$-root invariant. The following result holds for any prime.

Proposition 10.2. The $B P \wedge \widetilde{B P}$-root invariant of $\alpha_{i / j}$ is given by

$$
R_{B P \wedge \widetilde{B P}}\left(\alpha_{i / j}\right)=(-1)^{i-j} \widetilde{\beta}_{i / j}+p \widetilde{\beta}_{i / j} B P_{*} \widetilde{B P} .
$$

Corollary 10.3. Suppose the Greek letter element $\alpha_{i / j}$ exists and is a permanent cycle in the ANSS. Let $\bar{\alpha}_{i / j} \in \pi_{*}^{S}$ be the element that $\alpha_{i / j}$ detects. Then the zeroth filtered root invariant of $\bar{\alpha}_{i / j}$ is trivial. If $p$ is odd, the first filtered root invariant of $\bar{\alpha}_{i / j}$ is given by

$$
(-1)^{i-j} \widetilde{\beta}_{i / j}+c \cdot \widetilde{\alpha}_{i(p+1)+j} \in R_{B P}^{[1]}\left(\bar{\alpha}_{i / j}\right),
$$

where $c$ is some constant. The second filtered root invariant is given by

$$
(-1)^{i-j} \beta_{i / j} \in R_{B P}^{[2]}\left(\bar{\alpha}_{i / j}\right) .
$$

Deducing the first filtered root invariant in Corollary 10.3 from Proposition 10.2 amounts to computing the indeterminacy group $A$ described in Corollary 5.9. The second filtered root invariant then follows from Theorem 5.3. We describe a spectral sequence which computes $A$ (10.8). We fully compute $A$ for odd primes $p$ and describe some of the 2-primary aspects of this computation in Remark 10.6.

We have shown that $\alpha_{k} \dot{\in} R_{B P}^{[1]}\left(p^{k}\right)$. It is shown in [25], 35] that these elements survive in the Adams-Novikov spectral sequence to elements of $R\left(p^{k}\right)$. Similarly, we have shown that $\beta_{k} \dot{\in} R_{B P}^{[2]}\left(\bar{\alpha}_{k}\right)$. Again, in [25], 35] it is shown that these elements survive to elements of $R\left(\bar{\alpha}_{k}\right)$ for $p \geq 5$. Sadofsky goes further in [35] to show that $\beta_{p / 2} \in R\left(\bar{\alpha}_{p / 2}\right)$ for $p \geq 5$.

In general, we have shown that if $p^{k-1} \mid s$, then $\beta_{s / k} \dot{\in} R_{B P}^{[2]}\left(\alpha_{s / k}\right)$. According to the summary presented in [33, 5.5], the elements $\beta_{s / k}$ exist and are permanent cycles in the ANSS for $p \geq 5$ for these values of $k$ and $s$. However, without any additional information, we can only deduce the conclusions of Theorem 5.1, In Section 12 we indicate what these filtered root invariant calculations mean for the computation of homotopy root invariants in low dimensions at the prime 3 .

In proving Propositions 10.1 and 10.2, we shall need to make use of the following well-known computation (see Lemma 2.1 of [2]).

Lemma 10.4. Let $E$ be a complex-oriented spectrum whose associated p-series $[p]_{E}(x)$ is not a zero divisor in $E^{*}[[x]]$. Then the coefficient ring of the Tate spectrum is given by

$$
\pi_{*}(t E)=\left(E_{*}[[x]] /\left([p]_{E}(x)\right)\right)\left[x^{-1}\right]=E_{*}((x)) /\left([p]_{E}(x)\right),
$$

where the degree of $x$ is -2 . Furthermore, the inclusions of the projective spectra are described as the inclusion of the fractional ideal

$$
x^{N} E_{*}[[x]] /\left([p]_{E}(x) / x\right)=E_{*}\left(\Sigma(B \mathbb{Z} / p)^{-2 N}\right) \rightarrow \pi_{*}(t E)=E_{*}((x)) /\left([p]_{E}(x)\right) .
$$

According to Appendix B of [15, the skeletal and coskeletal filtrations of $(E \wedge P)_{-\infty}$ give rise to the same notions of the $E$-root invariant. It follows that 
$E$-root invariants are quite easy to compute for complex orientable spectra $E$ provided one has some knowledge of the $p$-series of $E$. The method is outlined in the following corollary.

Corollary 10.5. Suppose that $\alpha$ is an element of $E_{*}$. Viewing $\bar{\alpha}$ as the image of the constant power series $\alpha$ in $E_{*}((x)) /\left([p]_{E}(x)\right)$, suppose that $n$ is maximal so that

$$
\bar{\alpha}=a_{n} x^{n}+\mathcal{O}\left(x^{n+1}\right)
$$

with $a_{n} \in E_{*}$ non-zero. Then $a_{n}$ is an element of $R_{E}(\alpha)$.

Proof of Proposition 10.1. We first compute $R_{B P}\left(p^{i}\right)$. The $p$-series $[p]_{F}(x)$ of the universal $p$-typical formal group $F$ is given by

$$
[p](x)=p x+_{F} v_{1} x^{p}+{ }_{F} v_{2} x^{p^{2}}+{ }_{F} \cdots,
$$

where $v_{i}$ are the Araki generators of $B P_{*}$. In $t B P_{*}$, we have the relation

$$
p=-v_{1} x^{p-1}+\mathcal{O}\left(x^{p}\right)
$$

which gives, upon taking the $i^{\text {th }}$ power,

$$
p^{i}=\left(-v_{1}\right)^{i} x^{(p-1) i}+\mathcal{O}\left(x^{(p-1) i+1}\right) .
$$

Any other expression of $p^{i}$ in terms of $x^{(p-1) i}$ and higher order terms will have a leading coefficient that differs by an element of the ideal $p B P_{*} \subset B P_{*}$. Using Corollary 10.5 and Proposition 5.7 we may conclude that

$$
\left(-v_{1}\right)^{i}+p B P_{*}=R_{B P}\left(p^{i}\right) \supseteq R_{B P}^{[0]}\left(p^{i}\right) .
$$

We calculate the Adams-Novikov $d_{1}$ on this coset as

$$
d_{1}\left(v_{1}^{i}\right)=\eta_{R}\left(v_{1}^{i}\right)-v_{1}^{i} .
$$

The algebraic Greek letters $\alpha_{i}$ are defined by

$$
\alpha_{i}=\frac{\eta_{R}\left(v_{1}^{i}\right)-v_{1}^{i}}{p}
$$

so we are in the situation where

$$
d_{1}\left(R_{B P}^{[0]}\left(p^{i}\right)\right) \subseteq p \cdot(-1)^{i} \alpha_{i}+p^{2} \alpha_{i} B P_{*} B P .
$$

By Theorem [5.3. we may conclude that the first filtered root invariant $R_{B P}^{[1]}\left(p^{i}\right)$ is contained in the coset $(-1)^{i} \alpha_{i}+p \alpha_{i} B P_{*} B P$.

Proof of Proposition 10.2. The proof consists of two parts. In Part 1 we prove the proposition with $v_{1}$ inverted, and in Part 2 we prove that the $v_{1}^{-1} B P \wedge \widetilde{B P}$-root invariant can be lifted to compute the $B P \wedge \widetilde{B P}$-root invariant.

Part 1: computing the $v_{1}^{-1} B P \wedge \widetilde{B P}$-root invariant.

We may calculate $t B P \wedge \widetilde{B P}_{*}$ from $t B P_{*} B P$ using the split short exact sequence

$$
0 \rightarrow t B P_{*} \rightarrow t B P \wedge B P_{*} \rightarrow t B P \wedge \widetilde{B P}_{*} \rightarrow 0 .
$$

Applying Lemma 10.4 to the left and right complex orientations of $B P_{*} B P$, we get

$$
\begin{gathered}
t B P \wedge B P_{*}=B P \wedge B P_{*}\left(\left(x_{L}\right)\right) /\left([p]_{L}\left(x_{L}\right)\right) \\
B P \wedge B P_{*}\left(\left(x_{R}\right)\right) /\left([p]_{R}\left(x_{R}\right)\right) .
\end{gathered}
$$


Here, the $p$-series $[p]_{L}(x),[p]_{R}(x)$, and the coordinate $x_{R}$ are given by the formulas

$$
\begin{aligned}
{[p]_{L}(x) } & =p x+_{F_{L}} v_{1} x^{p}+_{F_{L}} v_{2} x^{p^{2}}+F_{F_{L}} \cdots, \\
{[p]_{R}(x) } & =p x+_{F_{R}} \eta_{R}\left(v_{1}\right) x^{p}+_{F_{R}} \eta_{R}\left(v_{2}\right) x^{p^{2}}+{ }_{F_{R}} \cdots, \\
x_{L} & =x_{R}+{ }_{F_{L}} t_{1} x_{R}^{p}+_{F_{L}} t_{2} x_{R}^{p^{2}}+_{F_{L}} \cdots .
\end{aligned}
$$

The formal group laws $F_{L}$ and $F_{R}$ are the $p$-typical formal group laws over $B P_{*} B P$ induced by left and right units $\eta_{L}$ and $\eta_{R}$, respectively. Consider the following computation in $t B P \wedge \widetilde{B P}_{*}$ :

$$
\begin{aligned}
p x_{R} & =[-1]_{F_{R}}\left(\eta_{R}\left(v_{1}\right) x_{R}^{p}+{ }_{F_{R}} \eta_{R}\left(v_{2}\right) x_{R}^{p^{2}}+F_{R} \cdots\right) \\
& =-\eta_{R}\left(v_{1}\right) x_{R}^{p}-\eta_{R}\left(v_{2}\right) x_{R}^{p^{2}}+\mathcal{O}\left(x_{R}^{p^{2}+1}\right) .
\end{aligned}
$$

The last equality for $p>2$ follows from the fact that for any $p$-typical formal group law $G$ where $p>2$, the -1 -series is given by

$$
[-1]_{G}(x)=-x \text {. }
$$

For $p=2$ this is not true, but it turns out that the last equality of Equation (10.2) still holds up to the power indicated in $t B P_{*} \widetilde{B P}$. The two expressions we want to be equal are written out explicitly as follows:

$$
\begin{aligned}
& {[-1]_{F_{R}}\left(\eta_{R}\left(v_{1}\right) x_{R}^{2}+F_{R} \eta_{R}\left(v_{2}\right) x_{R}^{4}+F_{R} \cdots\right)} \\
& \quad=\left(2 t_{1}-v_{1}\right) x_{R}^{2}+\left(14 t_{2}-4 t_{1}^{3}-v_{1} t_{1}^{2}-3 v_{1}^{2} t_{1}-v_{2}+v_{1}^{3}\right) x_{R}^{4}+\mathcal{O}\left(x_{R}^{5}\right)
\end{aligned}
$$

and

$$
\begin{aligned}
& -\eta_{R}\left(v_{1}\right) x_{R}^{2}-\eta_{R}\left(v_{2}\right) x_{R}^{4}+\mathcal{O}\left(x_{R}^{5}\right) \\
& \quad=\left(2 t_{1}-v_{1}\right) x_{R}^{2}+\left(14 t_{2}+4 t_{1}^{3}-13 v_{1} t_{1}^{2}+3 v_{1}^{2} t_{1}-v_{2}\right) x_{R}^{4}+\mathcal{O}\left(x_{R}^{5}\right) .
\end{aligned}
$$

The coefficient of $x_{R}^{2}$ is the same in Equations (10.3) and (10.4). Since we are working in $t B P_{*} \widetilde{B P}$, we are working modulo the 2 -series, and thus we only need the coefficients of $x_{R}^{4}$ to be equivalent modulo 2. However, the coefficient of $x_{R}^{4}$ in Equation (10.3) has an extra $v_{1}^{3}$, but since we are working in the reduced setting of $t B P_{*} \widetilde{B P}$, we have $v_{1} x_{L}^{4}=0$. We may switch to $x_{R}$ since $x_{L}^{4}=x_{R}^{4}+\mathcal{O}\left(x_{R}^{5}\right)$.

Returning to our manipulations of the $p$-series, upon dividing Equation (10.2) by $x_{R}$, we get

$$
p=-\eta_{R}\left(v_{1}\right) x_{R}^{p-1}-\eta_{R}\left(v_{2}\right) x_{R}^{p^{2}-1}+\mathcal{O}\left(x_{R}^{p^{2}}\right)
$$

which implies

$$
\eta_{R}\left(v_{1}\right)=-\eta_{R}\left(v_{2}\right) x_{R}^{p^{2}-p}+\mathcal{O}\left(x_{R}^{p^{2}-p+1}\right)
$$

since the image of $p$ is zero in $B P_{*} \widetilde{B P}$. Taking the $i^{\text {th }}$ power and exploiting the fact that the image of $v_{1}^{i}$ in $B P_{*} \widehat{B P}$ is also zero, we may write

$$
p^{j} \alpha_{i / j}=\eta_{R}\left(v_{1}\right)^{i}-v_{1}^{i}=\left(-\eta_{R}\left(v_{2}\right)\right)^{i} x_{R}^{i\left(p^{2}-p\right)}+\mathcal{O}\left(x_{R}^{i\left(p^{2}-p\right)+1}\right) .
$$

Since we are modding out by the $p$-series in $x_{L}$, we have the relation

$$
\begin{aligned}
p x_{L} & =[-1]_{F_{L}}\left(v_{1} x_{L}^{p}+F_{F_{L}} v_{2} x_{L}^{p^{2}}+F_{F_{L}} \cdots\right) \\
& =-v_{1} x_{L}^{p}+\mathcal{O}\left(x_{L}^{p^{2}}\right)
\end{aligned}
$$


or, dividing by $x_{L}$ and taking the $j^{\text {th }}$ power,

$$
p^{j}=\left(-v_{1}\right)^{j} x_{L}^{j(p-1)}+\mathcal{O}\left(x_{L}^{j(p-1)+p^{2}-p}\right) .
$$

Upon combining Equation (10.6) with Equation (10.5), and using the fact that $x_{R}^{k}=x_{L}^{k}+\mathcal{O}\left(x_{R}^{k+1}\right)$, our original expression for $\alpha_{i / j}$ becomes the following:

$$
\begin{array}{rl}
\left(-v_{1}\right)^{j} \alpha_{i / j}+\alpha_{i / j} & \mathcal{O}\left(x_{R}^{p^{2}-p}\right) \\
& =(-1)^{i}\left(\eta_{R}\left(v_{2}\right)^{i}-v_{2}^{i}\right) x_{R}^{i\left(p^{2}-p\right)-j(p-1)}+\mathcal{O}\left(x_{R}^{i\left(p^{2}-p\right)-j(p-1)+1}\right) .
\end{array}
$$

Explicit formulas (see, for instance, 33 , 4.3.21]) reveal that

$$
\eta_{R}\left(v_{2}\right)=v_{2}+v_{1} t_{1}^{p}-v_{1}^{p} t_{1}+p y
$$

for some $y \in B P_{*} B P$. Using this formula and Equation (10.6) to write elements which are divisible by $p$ in terms of higher order elements, we have

$$
\begin{aligned}
\left(-v_{1}\right)^{j} \alpha_{i / j}+\alpha_{i / j} \mathcal{O} & \left(x_{R}^{p^{2}-p}\right) \\
& =(-1)^{i} v_{1}^{j} \tilde{\beta}_{i / j} x_{R}^{i\left(p^{2}-p\right)-j(p-1)}+\mathcal{O}\left(x_{R}^{i\left(p^{2}-p\right)-j(p-1)+1}\right) .
\end{aligned}
$$

In order to solve for $\alpha_{i / j}$, we would like to divide by $v_{1}^{j}$. Therefore, we shall finish our algebraic manipulations in $t v_{1}^{-1} B P \wedge \widetilde{B P}_{*}$. We will then show that we can pull back our results to results in $t B P \wedge \widetilde{B P}_{*}$. Taking the image of Equation (10.7) in $t v_{1}^{-1} B P \wedge B P_{*}$ and dividing by $\left(-v_{1}\right)^{j}$, we get the expression

$$
\alpha_{i / j}=(-1)^{i-j} \widetilde{\beta}_{i / j} x_{R}^{i\left(p^{2}-p\right)-j(p-1)}+\alpha_{i / j} \mathcal{O}\left(x_{R}^{p^{2}-p}\right)+\mathcal{O}\left(x_{R}^{i\left(p^{2}-p\right)-j(p-1)+1}\right) .
$$

By successively substituting the left-hand side of this expression into the right-hand side, we obtain the expression

$$
\alpha_{i / j}=(-1)^{i-j} \tilde{\beta}_{i / j} x_{R}^{i\left(p^{2}-p\right)-j(p-1)}+\text { higher order terms. }
$$

We may conclude that $R_{v_{1}^{-1} B P \wedge \widetilde{B P}}\left(\alpha_{i / j}\right)=\widetilde{\beta}_{i / j}$, or that the $v_{1}^{-1} B P \wedge \widetilde{B P}$-root invariant lives in a higher stem.

Part 2: lifting to the $B P \wedge \widetilde{B P}$-root invariant.

We claim that the localization map

$$
B P \wedge \widetilde{B P}_{*}\left(P_{-N}\right) \rightarrow v_{1}^{-1} B P \wedge \widetilde{B P}_{*}\left(P_{-N}\right)
$$

is an inclusion. In order to see this, chase the following diagram:

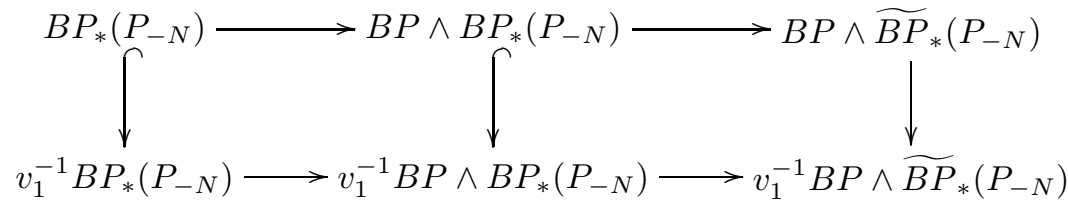

The relevant observations are that the other two localization maps in the diagram are inclusions (since there is no $v_{1}$ torsion in these groups), and the top and bottom sequences are compatibly split cofiber sequences. 
We wish to deduce something about the $B P \wedge \widetilde{B P}$-root invariant from the computation of the $v_{1}^{-1} B P \wedge \widetilde{B P}$-root invariant. We refer to the following diagram:

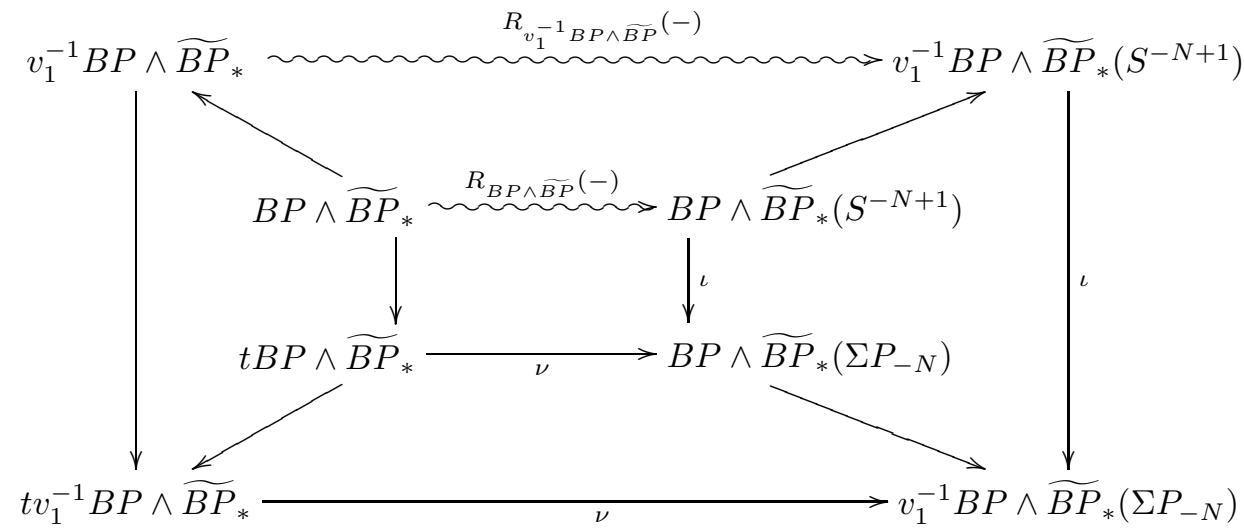

Here $N$ equals $2 i\left(p^{2}-p\right)-2 j(p-1)+1$. We have shown that $\iota\left((-1)^{i-j} \widetilde{\beta}_{i / j}\right)=$ $\nu\left(\alpha_{i / j}\right)$ after $v_{1}$ is inverted. Because the $v_{1}$-localization map is an inclusion, we may conclude that $\iota\left((-1)^{i-j} \widetilde{\beta}_{i / j}\right)=\nu\left(\alpha_{i / j}\right)$ in $B P \wedge \widetilde{B P}_{*}\left(\Sigma P_{-N}\right)$. Therefore, we have computed the $B P_{*} \widetilde{B P}$-root invariant

$$
R_{B P \wedge \widetilde{B P}}\left(\alpha_{i / j}\right)=(-1)^{i-j} \widetilde{\beta}_{i / j}+p \widetilde{\beta}_{i / j} B P_{*} \widetilde{B P}
$$

or it lies in a larger stem. The latter cannot be the case, however, as $\iota\left(\widetilde{\beta}_{i / j}\right)$ is non-zero.

We wish to apply Corollary 5.9 to Proposition 10.2 and conclude that

$$
R_{B P}^{[1]}\left(\bar{\alpha}_{i / j}\right) \subseteq(-1)^{i-j} \widetilde{\beta}_{i / j}+p \widetilde{\beta}_{i / j} B P_{*} \widetilde{B P}+A .
$$

Here $A$ is the image of the boundary homomorphism

$$
\pi_{i q-1}\left(W_{0}^{(0,1)}\left(P_{(-(i p-j) q-1,-(i p-j) q)}\right)\right) \stackrel{\partial}{\rightarrow} \pi_{i q-2}\left(W_{1}^{1}\left(S^{-(i p-j) q-1}\right)\right) .
$$

Let $N$ be $(i p-j) q+1$.

The group $\pi_{*}\left(W_{0}^{(0,1)}\left(P_{(-N,-N+1)}\right)\right)$ may be computed from the AHSS associated to the filtration

$$
W_{0}^{0}\left(S^{-N}\right) \subset W_{0}^{(0,1)}\left(P_{(-N,-N+1)}^{-N+1}\right) \subset W_{0}^{(0,1)}\left(P_{(-N,-N+1)}^{-N+2}\right) \subset \cdots .
$$

The resulting spectral sequence takes the form

$$
E_{1}^{k, l}=\left\{\begin{array}{lll}
0, & l \neq \equiv 0,-1 \quad(\bmod q), \\
\pi_{k}\left(W_{0}^{1}\left(S^{l}\right)\right), & l \equiv 0,-1 \quad(\bmod q), l>-N, \\
\pi_{k}\left(W_{0}^{0}\left(S^{-N}\right)\right), & l=-N,
\end{array}\right.
$$

and converges to $\pi_{k}\left(W_{0}^{(0,1)}\left(P_{(-N,-N+1)}\right)\right)$. The groups $\pi_{*}\left(W_{0}^{1}\left(S^{l}\right)\right)$ in the $E_{1}$-term of the spectral sequence (10.8) may be computed by taking the 0- and 1-lines of the $E_{1}$-term of the ANSS for the sphere, and taking the cohomology with respect to the $d_{1}$ from the 0 -line to the 1-line. The differentials in spectral sequence (10.8) are a restriction of the differentials in the AAHSS. 
The image of the map $\partial$ is generated by the image of Adams-Novikov $d_{1}$ 's supported on the 0-line, the subgroup $p W_{1}^{1}\left(S^{-N}\right)$, and the images of the higher differentials in the AAHSS whose sources are permanent cycles in spectral sequence (10.8) and whose targets are elements in Adams-Novikov filtration 1 that are carried by the $-N$-cell.

We remark that for $0 \neq x \in \pi_{*}\left(W_{1}^{1}\left(S^{0}\right)\right)$ which is not a permanent cycle, we have a non-trivial differential

$$
d_{1}(x[k q])=p x[k q-1] .
$$

The reason the differential must be non-trivial is that there is no torsion in the $E_{1}$-term of the ANSS, and if $p x$ were the target of a $d_{1}$ in the ANSS, then $x$ would have to be a $d_{1}$-cycle. Thus, if we are looking for longer differentials in spectral sequence (10.8) supported by $k q$-cells, we may restrict our search to those which are actually $d_{1}$-cycles.

We now use spectral sequence (10.8) to compute the indeterminacy group $A$ for odd primes $p$.

Proof of Corollary 10.3. Since filt ${ }_{B P}\left(\bar{\alpha}_{i / j}\right)=1$, Lemma 5.6 implies the filtered root invariant $R_{B P}^{[0]}\left(\bar{\alpha}_{i / j}\right)$ is trivial. In Proposition [10.2, we found the $B P \wedge \widetilde{B P}$ root invariants $R_{B P \wedge \widetilde{B P}}\left(\alpha_{i / j}\right)$. We will now deduce the first filtered root invariant $R_{B P}^{[1]}\left(\bar{\alpha}_{i / j}\right)$ through the application of Proposition [5.8. We just need to compute $A$ using spectral sequence (10.8).

The indeterminacy group $A$ is generated by the images of Adams-Novikov $d_{1}$ 's, the subgroup $p W_{1}^{1}\left(S^{-N}\right)$, and the higher AAHSS differentials. We just need to compute the latter. The only elements of spectral sequence (10.8) that could contribute to $A$ are those of the form

$$
\alpha_{k / l}[(i-k) q], \quad k \leq i p-j .
$$

Proposition 8.5 tells us that only one can contribute to $\delta$ and that contribution is given by

$$
\partial\left(\alpha_{i(p+1)-j-l}[-(i p-j-l) q]\right)=\widetilde{\alpha}_{i(p+1)-j}[-(i p-j) q-1],
$$

where $l=\nu_{p}(i)+1$. Thus $A$ is also spanned by the element $\widetilde{\alpha}_{-i(p+1)+j}$. We conclude that

$$
(-1)^{i-j} \widetilde{\beta}_{i / j}+c \cdot \widetilde{\alpha}_{-i(p+1)+j} \in R_{B P}^{[1]}\left(\bar{\alpha}_{i / j}\right),
$$

where $c$ is some constant.

To prove the second part of the proposition we appeal to Theorem 5.3 , There is an Adams-Novikov differential $d_{1}\left(\widetilde{\beta}_{i / j}\right)=p \cdot \beta_{i / j}$. The filtered root invariant $R_{B P}^{[1]}\left(\alpha_{i / j}\right)$ is carried by the $-N_{1}$-cell, where $N_{1}=2\left(i\left(p^{2}-p\right)-j(p-1)\right)+1=$ $(i p-j) q+1$. The first cell to attach non-trivially to this cell is the $-(i p-j) q$-cell, and this is by the degree $p$ map. We may conclude that

$$
(-1)^{i-j} \beta_{i / j} \in R_{B P}^{[2]}\left(\bar{\alpha}_{i / j}\right) \text {. }
$$

Remark 10.6. Computing the group $A$ at the prime 2 requires a more careful analysis. The AAHSS differentials do not follow immediately from the $J$-spectrum AHSS 
differentials computed in 23, because of the varying Adams-Novikov filtrations of the $v_{1}$-periodic elements. For instance, it turns out that

$$
\alpha_{4 / 4}=\widetilde{\beta}_{2 / 2}+x_{7} \in R_{B P}^{[1]}\left(\bar{\alpha}_{2 / 2}\right),
$$

where

$$
x_{7} \equiv v_{2} t_{1}+v_{1}\left(t_{2}+t_{1}^{3}\right) \quad(\bmod 2) .
$$

If $i=j=1$, then $\widetilde{\beta}_{1}$ is a permanent cycle which represents the element $\alpha_{2 / 2}$. If $i=j=2$, then $\widetilde{\beta}_{2 / 2}+x_{7}$ is a permanent cycle which represents the element $\alpha_{4 / 4}$. Low-dimensional calculations seem to indicate that in all other circumstances we have

$$
\beta_{i / j}+c \cdot \widetilde{\alpha}_{3 i-j+1} \alpha_{1} \in R_{B P}^{[2]}\left(\bar{\alpha}_{i / j}\right)
$$

where $c$ is some constant which may or may not be zero and $\widetilde{\alpha}_{k}$ is equal to $\alpha_{i / j}$ with $j$ maximal. The anomalous cases with $i=j=1,2$ correspond to the existence of the 'extra' Hopf invariant 1 elements $\nu$ and $\sigma$, which are detected in the ANSS by $\alpha_{2 / 2}$ and $\alpha_{4 / 4}$, respectively. These filtered root invariants are thus consistent with the homotopy root invariant, which takes each Hopf invariant 1 element to the next one, if it exists.

\section{Computation of $R\left(\beta_{1}\right)$ at odd primes}

In this section we will compute the root invariant of $\beta_{1}$ at odd primes. This result was stated, but not proved, in 25 . We do this by first computing the $B P$-filtered root invariants, and then by executing Step 4 of Procedure 9.1 .

Proposition 11.1. For $p>2$, the top filtered root invariant of $\beta_{1}$ is given by $R_{B P}^{[2 p]}\left(\beta_{1}\right) \doteq \beta_{1}^{p}$.

Sketch of Proof. We first wish to show that $\beta_{p / p} \in R_{B P}^{[2]}\left(\beta_{1}\right)$. In [33], it is shown that modulo the ideal $I_{1}$, the representatives for $\beta_{1}$ and $\beta_{p / p}$ in the cobar complex are given by

$$
\begin{aligned}
\beta_{1} & \equiv-\frac{1}{p} \sum_{0<i<p}\left(\begin{array}{c}
p \\
i
\end{array}\right) t_{1}^{i} \mid t_{1}^{p-i} \quad\left(\bmod I_{1}\right), \\
\beta_{p / p} & \equiv-\frac{1}{p} \sum_{0<i<p^{2}}\left(\begin{array}{c}
p^{2} \\
i
\end{array}\right) t_{1}^{i} \mid t_{1}^{p^{2}-i} \quad\left(\bmod I_{1}\right) .
\end{aligned}
$$

Now, $\nu_{p}\left(\begin{array}{c}p^{2} \\ i\end{array}\right)=1$ if and only if $p \mid i$. Therefore, the expression for $\beta_{p / p}$ modulo $I_{1}$ may be simplified as

$$
\beta_{p / p} \equiv-\frac{1}{p} \sum_{0<i<p}\left(\begin{array}{c}
p^{2} \\
i p
\end{array}\right) t_{1}^{i p} \mid t_{1}^{p(p-i)} \quad\left(\bmod I_{1}\right) .
$$

Since $\left(\begin{array}{l}p \\ i\end{array}\right) \equiv\left(\begin{array}{c}p^{2} \\ p i\end{array}\right)(\bmod p)$ for $0<i<p$, we see that in $\operatorname{Ext}_{B P_{*} B P / I_{1}}\left(B P_{*}\right)$, the element $\beta_{p / p}$ is obtained from $\beta_{1}$ by application of the $0^{\text {th }}$ algebraic Steenrod operation. By computing the reduction map

$$
\operatorname{Ext}_{B P_{*} B P}\left(B P_{*}\right) \rightarrow \operatorname{Ext}_{B P_{*} B P / I_{1}}\left(B P_{*} / I_{1}\right)
$$

we may conclude that

$$
P^{0}\left(\beta_{1}\right) \equiv \beta_{p / p} \quad(\bmod p)
$$


in $\operatorname{Ext}_{B P_{*} B P}\left(B P_{*}\right)$. By an algebraic analog of Jones's Kahn-Priddy theorem, this coincides with the $B P$-algebraic root invariant, which corresponds to the first nontrivial filtered root invariant. Thus we have the filtered root invariant

$$
\beta_{p / p} \in R_{B P}^{[2]}\left(\beta_{1}\right) .
$$

In the ANSS, the element $\beta_{p / p}$ supports the Toda differential

$$
d_{q+1}\left(\beta_{p / p}\right) \doteq \beta_{1}^{p} \alpha_{1} \text {. }
$$

The $-\left(p^{2}-p-1\right) q-1$-cell attaches to the $-\left(p^{2}-p\right) q-1$-cell of $P_{-\infty}$ with attaching map $\alpha_{1}$. Therefore, Theorem 5.3 tells us that

$$
\beta_{1}^{p} \dot{\in} R_{B P}^{[2 p]}\left(\beta_{1}\right) .
$$

There is no room for indeterminacy in the ANSS.

Corollary 11.2. For $p>2$, the homotopy root invariant of $\beta_{1}$ is given by

$$
R\left(\beta_{1}\right) \doteq \beta_{1}^{p} .
$$

Proof. The element $\beta_{1}^{p}$ lies on the Adams-Novikov vanishing line, so it must be the top filtered root invariant. Therefore, we may apply Corollary 5.2 to see that either the image of the element $\beta_{1}^{p}$ under the inclusion of the $\left(-\left(p^{2}-p-1\right) q-1\right)$-cell of $P_{-\left(p^{2}-p-1\right) q-1}$ is null, or $\beta_{1}^{p}$ actually detects the homotopy root invariant. We must therefore show that the element $\beta_{1}^{p}\left[-\left(p^{2}-p-1\right) q-1\right]$ in the AHSS for $P_{-\left(p^{2}-p-1\right) q-1}$ is not the target of a differential. We will actually show that $\beta_{1}^{p}\left[-\left(p^{2}-p-1\right) q-1\right]$ is not the target of a differential in the AAHSS, and that there are no possible sources of differentials in the ANSS for $P_{-\left(p^{2}-p-1\right) q-1}$ with target $\beta_{1}^{p}\left[-\left(p^{2}-p-1\right) q-1\right]$.

According to the low-dimensional computations of the ANSS at odd primes given in [33, Ch. 4], the only elements in the $E_{1}$-term of the AAHSS which can kill $\beta_{1}^{p}\left[-\left(p^{2}-p-1\right) q-1\right]$ in either the AAHSS or the ANSS are the elements

$$
\begin{aligned}
\beta_{k}[-(k-1)(p+1) q], & 1 \leq k<p, \\
\alpha_{k / l}[-(k-p) q-1], & 1 \leq k<p^{2}, 1 \leq l \leq \nu_{p}(k)+1,
\end{aligned}
$$

as well as elements in $\alpha_{1}-\beta_{1}$ towers, i.e. those that satisfy the hypotheses of Proposition 8.4. These latter elements cannot kill $\beta_{1}^{p}\left[-\left(p^{2}-p-1\right) q-1\right]$ in the AAHSS and cannot survive to kill anything in the ANSS by Proposition 8.4. Some care must be taken at $p=3$, but in this low-dimensional range there are no deviations from this pattern.

By Proposition 8.3 , the elements $\beta_{k}[-(k-1)(p+1) q]$ support non-trivial AAHSS differentials

$$
\left.d_{2}\left(\beta_{k}[-(k-1)(p+1) q]\right) \doteq \alpha_{1} \cdot \beta_{k}[-k(p+1) q]\right) .
$$

According to Proposition 8.5 for $l<\nu_{p}(k)+1$, we have differentials in the AAHSS

$$
d_{1}\left(\alpha_{k / l+1}[-(k-p) q]\right) \doteq \alpha_{k / l}[-(k-p) q-1]
$$

whereas for $l=\nu_{p}(k)+1$ and $k \geq 3$ we have

$$
d_{5}\left(\alpha_{k-2}[-(k-p-2) q]\right) \doteq \alpha_{k / l}[-(k-p) q-1] .
$$

For $k=2$ we have 38

$$
d_{4}(1[p q-1])=\alpha_{2}[(p-2) q-1] .
$$


Finally, Proposition 8.3 implies that

$$
d_{q}\left(\alpha_{1}[(p-1) q-1]\right) \doteq \beta_{1}[-1] .
$$

There are no elements left to kill $\beta_{1}^{p}\left[-\left(p^{2}-p-1\right) q-1\right]$.

\section{LOW-DIMENSIONAL COMPUTATIONS OF ROOT INVARIANTS AT $p=3$}

The aim of this section is to use knowledge of the ANSS for $\pi_{*}^{S}$ in the first 100 stems to compute the homotopy root invariants of some low-dimensional Greek letter elements $\alpha_{i / j}$ at $p=3$. These results are summarized in the following proposition.

Proposition 12.1. We have the following root invariants at $p=3$ :

$$
\begin{aligned}
R\left(\alpha_{1}\right) & =\beta_{1}, \\
R\left(\alpha_{2}\right) & \doteq \beta_{1}^{2} \alpha_{1}, \\
R\left(\alpha_{3 / 2}\right) & =-\beta_{3 / 2}, \\
R\left(\alpha_{3}\right) & =\beta_{3}, \\
R\left(\alpha_{4}\right) & \doteq \beta_{1}^{5}, \\
R\left(\alpha_{5}\right) & =\beta_{5}, \\
R\left(\alpha_{6 / 2}\right) & =\beta_{6 / 2}, \\
R\left(\alpha_{6}\right) & =-\beta_{6} .
\end{aligned}
$$

It is interesting to note that although $\beta_{2}$ exists, it fails to be the root invariant of $\alpha_{2}$. The element $\beta_{4}$ does not exist, so it cannot be the root invariant of $\alpha_{4}$. In Section 15 we will prove that $\beta_{i} \dot{\in} R\left(\alpha_{i}\right)$ for $i \equiv 0,1,5(\bmod 9)$. The remainder of this section is devoted to proving Proposition 12.1.

Figure 5 shows the Adams-Novikov $E_{2}$ term. These charts were created from the computations in 33. Solid lines represent multiplication by $\alpha_{1}$ and dotted lines represent the Massey product $\left\langle-, \alpha_{1}, \alpha_{1}\right\rangle$. Dashed lines represent hidden extensions.

In Section 10, we proved in Corollary 10.3 that we have filtered root invariants

$$
(-1)^{i-j} \beta_{i / j} \in R^{[2]}\left(\alpha_{i / j}\right) .
$$

We supplement those results with two higher-filtered root invariants particular to $p=3$.

Proposition 12.2. We have the following higher-filtered root invariant of $\alpha_{2}$ :

$$
\beta_{1}^{2} \alpha_{1} \dot{\in} R_{B P}^{[5]}\left(\alpha_{2}\right) .
$$

Proof. We know by Corollary 10.3 that

$$
-\beta_{2} \in R_{B P}^{[2]}\left(\alpha_{2}\right) \text {. }
$$

The element $\beta_{2}$ is a permanent cycle in the ANSS, which detects an element $\bar{\beta}_{2} \in$ $\pi_{*}^{S}$. We shall apply Theorem 5.4 to determine the higher-filtered root invariant from the following hidden Toda bracket:

$$
\beta_{1}^{3} \doteq\left\langle\alpha_{1}, 3, \bar{\beta}_{2}\right\rangle
$$



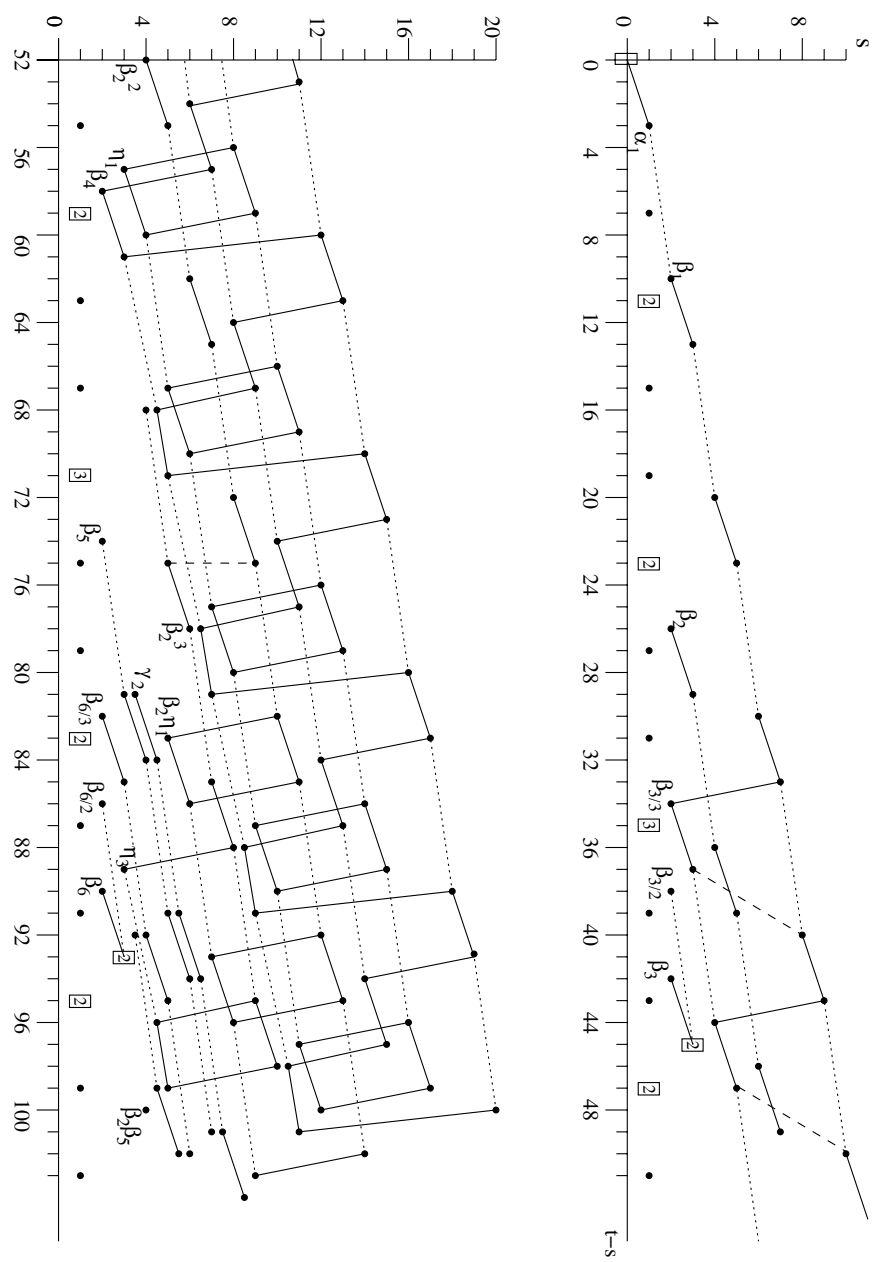

Figure 5. The Adams-Novikov spectral sequence at $p=3$

(see, for example, [33). Considering the attaching map structure of $P_{-\infty}$ we have the following equalities of Toda brackets (the first is an equality of homotopy Toda brackets whereas the second is an equality of Toda brackets in the ANSS):

$$
\begin{gathered}
\left\langle P_{-25}^{-20}\right\rangle\left(\bar{\beta}_{2}\right) \doteq\left\langle\alpha_{1}, 3, \bar{\beta}_{2}\right\rangle \doteq \beta_{1}^{3}, \\
\left\langle P_{-25}^{-17}\right\rangle\left(\beta_{1}^{2} \alpha_{1}\right) \doteq\left\langle\alpha_{1}, \alpha_{1}, \beta_{1}^{2} \alpha_{1}\right\rangle \doteq \beta_{1}^{3} .
\end{gathered}
$$

We conclude from Theorem 5.4(3) that

$$
\beta_{1}^{2} \alpha_{1} \dot{\in} R_{B P}^{[5]}\left(\alpha_{2}\right) .
$$

Proposition 12.3. We have the following higher-filtered root invariants of $\alpha_{4}$ :

$$
\begin{array}{r}
\beta_{3 / 3} \beta_{1}^{2} \dot{\in} R_{B P}^{[6]}\left(\alpha_{4}\right), \\
\beta_{1}^{5} \dot{\in} R_{B P}^{[10]}\left(\alpha_{4}\right) .
\end{array}
$$


Proof. We know from Corollary 10.3 that

$$
-\beta_{4} \in R_{B P}^{[2]}\left(\alpha_{4}\right)
$$

but $\beta_{4}$ is not a permanent cycle. There are Adams-Novikov differentials

$$
\begin{aligned}
d_{5}\left(\beta_{4}\right) & \doteq \beta_{3 / 3} \beta_{1}^{2} \alpha_{1}, \\
d_{5}\left(\beta_{3 / 3} \beta_{1}^{2}\right) & \doteq \beta_{1}^{5} \alpha_{1} .
\end{aligned}
$$

The higher-filtered root invariants of $\alpha_{4}$ are derived from two applications of Theorem 5.3. The relevant Toda brackets are computed below:

$$
\begin{gathered}
\left\langle P_{-40}^{-36}\right\rangle\left(\beta_{3 / 3} \beta_{1}^{2}\right) \doteq \alpha_{1} \cdot \beta_{3 / 3} \beta_{1}^{2}, \\
\left\langle P_{-44}^{-40}\right\rangle\left(\beta_{1}^{5}\right) \doteq \alpha_{1} \cdot \beta_{1}^{5} .
\end{gathered}
$$

We now apply Procedure 9.1 to our filtered root invariants. The results of executing Steps 1-3 in Procedure 9.1 are summarized in Table 2. The meanings of the contents of the columns are described below.

Element: contains the element $\alpha$ which we wish to calculate the root invariant of.

$\mathbf{R}^{[\mathbf{k}]}$ : indicates the $k^{\text {th }} B P$-filtered root invariant $\beta$ of $\alpha$. We would like $\beta$ to detect an element of $R(\bar{\alpha})$.

Cell: indicates the cell of $P_{-\infty}$ that carries the filtered root invariant.

$\left\{\gamma_{\mathbf{i}}\left[\mathbf{n}_{\mathbf{i}}\right]\right\}$ : contains the list of elements of the ANSS which could survive to the difference between the filtered root invariant and the homotopy root invariant. This is the list described in Step 1 of Procedure 9.1.

Diffs: indicates the differentials in the AAHSS which kill the elements $\gamma_{i}\left[n_{i}\right]$, as described in Step 2 of Procedure 9.1 or the differentials supported by the $\gamma_{i}\left[n_{i}\right]$, as described in Step 3 of Procedure 9.1, If the element $\gamma_{i}$ supports a non-trivial differential in the ANSS, we shall place a "(1)" to indicate that it is not a permanent cycle, and so cannot be a homotopy root invariant. If the $\gamma_{i}\left[n_{i}\right]$ satisfies the conditions of Proposition 8.4, then Steps 2 and 3 of Procedure 9.1 are automatically satisfied, as explained in Remark 9.2. We shall indicate this with a "(2)".

Some care must be taken in the case of $\alpha_{3}$. If $\left\langle\alpha_{1}, 3, \beta_{3}\right\rangle$ is non-zero (i.e. contains $\pm \beta_{2} \beta_{1}^{2}$ ), then the AAHSS differential

$$
d_{4}\left(\beta_{2} \beta_{1} \alpha_{1}[-7 q-1]\right) \doteq \beta_{2} \beta_{1}^{2}[-9 q-1]
$$

may be trivial in the AHSS with an intervening differential supported by $\beta_{3}[-8 q]$ (see Remark 9.2). This does not happen, though, as the Toda bracket $\left\langle\alpha_{1}, 3, \beta_{3}\right\rangle$ is actually zero. It is easily seen to have zero indeterminacy. If the Toda bracket were non-trivial, it would have to contain $\pm \beta_{2} \beta_{1}^{2}$, and thus $\alpha_{1} \cdot\left\langle\alpha_{1}, 3, \beta_{3}\right\rangle$ would be non-zero. This cannot be the case, since we have

$$
\alpha_{1} \cdot\left\langle\alpha_{1}, 3, \beta_{3}\right\rangle=-\left\langle\alpha_{1}, \alpha_{1}, 3\right\rangle \cdot \beta_{3}=\alpha_{2} \cdot \beta_{3}=0 .
$$

Now we must complete Step 4 of Procedure 9.1. We must determine that the filtered root invariants survive to non-trivial elements in the AHSS, and subsequently in the ANSS. For this we must determine which elements in the AAHSS 
TABle 2. Steps $1-3$ of Procedure 9.1 at $p=3$

\begin{tabular}{|c|c|c|c|c|}
\hline Element & $R^{|k|}$ & Cell & $\left\{\gamma_{i}\left[n_{i}\right]\right\}$ & Diffs \\
\hline$\alpha_{1}$ & $\overline{\beta_{1}}$ & $\overline{-2 q}$ & - & $\overline{-}$ \\
\hline$\alpha_{2}$ & $\beta_{1}^{2} \alpha_{1}$ & $-4 q-1$ & - & E \\
\hline$\alpha_{3 / 2}$ & $\beta_{3 / 2}$ & $-7 q$ & - & - \\
\hline$\alpha_{3}$ & $\beta_{3}$ & $-8 q$ & $\overline{\beta_{2} \beta_{1} \alpha_{1}[-7 q-1]}$ & $\overline{(2)}$ \\
\hline$\alpha_{4}$ & $\overline{\beta_{1}^{5}}$ & $-9 q$ & - & - \\
\hline$\alpha_{5}$ & $\beta_{5}$ & $\overline{-14 q}$ & $\begin{array}{c}\beta_{4} \beta_{1} \alpha_{1}[-13 q-1] \\
\eta_{1} \beta_{1}[-12 q-1] \\
\eta_{1} \beta_{1} \alpha_{1}[-13 q] \\
\beta_{2}^{2} \beta_{1}[-11 q] \\
\beta_{3 / 3} \beta_{1}^{3} \alpha_{1}[-12 q-1] \\
\beta_{2} \beta_{1}^{3} \alpha_{1}[-10 q-1] \\
\beta_{2} \beta_{1}^{4}[-12 q] \\
\beta_{1}^{6} \alpha_{1}[-11 q-1] \\
\beta_{1}^{7}[-13 q]\end{array}$ & $\begin{array}{l}(1) \\
(1) \\
(1) \\
(2) \\
(1) \\
(2) \\
(2) \\
(2) \\
(2)\end{array}$ \\
\hline$\alpha_{6 / 2}$ & $\beta_{6 / 2}$ & $-16 q$ & $\begin{array}{c}\beta_{2} \eta_{1}[-15 q-1] \\
\beta_{2}^{3} / \alpha_{1}[-13 q-1] \\
\beta_{4} \beta_{1} \alpha_{1}[-12 q-1] \\
\beta_{2}^{3}[-14 q] \\
\eta_{1} \beta_{1} \alpha_{1}[-12 q] \\
\beta_{2}^{2} \beta_{1}^{2} \alpha_{1}[-13 q-1] \\
\beta_{2}^{2} \beta_{1}^{3}[-15 q] \\
\beta_{3} / 3 \beta_{1}^{4}[-13 q] \\
\beta_{2} \beta_{1}^{5} \alpha_{1}[-14 q-1] \\
\beta_{1}^{7}[-12 q] \\
\beta_{1}^{8} \alpha_{1}[-15 q-1]\end{array}$ & $\begin{array}{l}(1) \\
(2) \\
(1) \\
(2) \\
(1) \\
(2) \\
(2) \\
(2) \\
(2) \\
(2) \\
(2)\end{array}$ \\
\hline$\alpha_{6}$ & $\beta_{6}$ & $-17 q$ & $\begin{array}{c}\eta_{1} \beta_{2}[-15 q-1] \\
\beta_{2}^{3} / \alpha_{1}[-13 q-1] \\
\beta_{4} \beta_{1} \alpha_{1}[-12 q-1] \\
\eta_{1} \beta_{2} \alpha_{1}[-16 q] \\
\beta_{2}^{3}[-14 q] \\
\eta_{1} \beta_{1} \alpha_{1}[-12 q] \\
\eta_{1} \beta_{1}^{3}[-16 q-1] \\
\beta_{2}^{2} \beta_{1}^{2} \alpha_{1} \\
\beta_{2}^{2} \beta_{1}^{3}[-15 q] \\
\beta_{3 / 3} \beta_{1}^{4}[-13 q] \\
\beta_{3 / 3} \beta_{1}^{5} \alpha_{1}[-16 q-1] \\
\beta_{2} \beta_{1}^{5} \alpha_{1}[-14 q-1] \\
\beta_{2} \beta_{1}^{6}[-16 q] \\
\beta_{1}^{8} \alpha_{1}[-15 q-1]\end{array}$ & $\begin{array}{l}(1) \\
(2) \\
(1) \\
(1) \\
(2) \\
(1) \\
(1) \\
(2) \\
(2) \\
(1) \\
(2) \\
(2) \\
(2) \\
(2)\end{array}$ \\
\hline
\end{tabular}

(or subsequently in the ANSS) can support differentials which kill the filtered root invariants.

Proposition 8.7 implies that for all of the $\alpha_{i / j}$ above whose filtered root invariants were $\beta_{i / j}$, the image of $\beta_{i / j}$ in the appropriate stunted projective space is non-trivial. 
Thus we conclude that

for $i \leq 6$ and $i \neq 2,4$.

$$
\beta_{i / j} \dot{\epsilon} R\left(\alpha_{i / j}\right)
$$

Step 4 of Procedure 9.1 is completed for $\alpha_{2}$ and $\alpha_{4}$ in the following lemmas. In both of these lemmas, we denote by $\bar{x}$ the element in homotopy detected by the ANSS element $x$. There is no ambiguity arising from higher Adams-Novikov filtration for the elements that we will be considering.

Lemma 12.4. The element $\overline{\beta_{1}^{2} \alpha_{1}}[-4 q-1]$ is not the target of a differential in the AHSS for $P_{-4 q-1}$.

Proof. Differentials which could kill $\overline{\beta_{1}^{2} \alpha_{1}}[-4 q-1]$ must have their source in the 7-stem. Proposition 8.5 demonstrates that in the targets of any AHSS differentials supported by $I m J$ elements in the 7 -stem are also $I m J$ elements. In the range we are considering, there is no room for shorter differentials. The only element left which could kill $\overline{\beta_{1}^{2} \alpha_{1}}[-4 q-1]$ is $\overline{\beta_{1}^{2}}[-3 q-1]$. However, the complex $P_{-4 q-1}^{-3 q-1}$ is reducible, so there can be no such differential.

Lemma 12.5. The element $\overline{\beta_{1}^{5}}[-9 q]$ is not the target of a differential in the AHSS for $P_{-9 q}$.

Proof. Exactly as in the proof of Lemma 12.4, the differentials supported by ImJ elements in the 15-stem can only hit other $\operatorname{ImJ}$ elements. The only elements left which can support differentials are

$$
\begin{array}{ccc}
\overline{\beta_{1}^{2}}[-q-1] \quad \overline{\beta_{1}^{2} \alpha_{1}}[-2 q] \quad \overline{\beta_{2} \beta_{1}}[-5 q-1], \\
\overline{\beta_{1}^{4}}[-6 q-1] \quad \overline{\beta_{3 / 3} \beta_{1} \alpha_{1}}[-8 q] .
\end{array}
$$

All but $\overline{\beta_{3 / 3} \beta_{1} \alpha_{1}}[-8 q]$ are the target or source of the AHSS $d_{2}$ differentials displayed as follows:

$$
\begin{aligned}
& \overline{\beta_{1}^{2}}[-q-1] \longmapsto \longmapsto \overline{\beta_{1}^{2} \alpha_{1}}[-2 q-1] \\
& \overline{\beta_{1}^{2}}[-q] \longmapsto \overline{\beta_{1}^{2} \alpha_{1}}[-2 q] \\
& \overline{\beta_{2} \beta_{1}}[-5 q-1] \longmapsto \overline{\beta_{2} \beta_{1} \alpha_{1}}[-6 q-1] \\
& \overline{\beta_{3 / 3} \alpha_{1}}[-5 q-1] \longmapsto \overline{\beta_{1}^{4}}[-6 q-1]
\end{aligned}
$$

The last of these is the result of a hidden $\alpha_{1}$-extension in the ANSS. The remaining element $\overline{\beta_{3 / 3} \beta_{1} \alpha_{1}}[-8 q]$ cannot support a differential whose target is $\overline{\beta_{1}^{5}}[-9 q]$, because $P_{-9 q}^{-8 q}$ is coreducible.

\section{Algebraic Filtered RoOt invariants}

In this section we will describe the Mahowald spectral sequence (MSS), which is a spectral sequence that computes $\operatorname{Ext}\left(H_{*} X\right)$ by applying $\operatorname{Ext}\left(H_{*}-\right)$ to an $E$ Adams resolution of $X$. This spectral sequence is described in [28]. We will briefly recall its construction for the reader's convenience. We will then define algebraic $E$-filtered root invariants, and indicate how some of the results of Section 5 carry over to the algebraic setting to compute algebraic root invariants from the algebraic filtered root invariants in the MSS. 
Let $E$ be a ring spectrum and suppose that $H$ is an $E$-ring spectrum in the sense that there is a map of ring spectra $E \rightarrow H$. Suppose furthermore that $H_{*} H$ is a flat $H_{*}$ module. Let $X$ be a (finite) spectrum. By applying $H_{*}$ to the cofiber sequences that make up the $E$-Adams resolution, we obtain short exact sequences

$$
0 \rightarrow H_{*}\left(W_{s}(X)\right) \rightarrow H_{*}\left(W_{s}^{s}(X)\right) \rightarrow H_{*}\left(\Sigma W_{s+1}(X)\right) \rightarrow 0
$$

which are split by the $E$-action map

$$
H \wedge W_{s}^{s}(X)=H \wedge E \wedge \bar{E}^{(s)} \wedge X \rightarrow H \wedge \bar{E}^{(s)} \wedge X=H \wedge W_{s}(X) .
$$

Thus the short exact sequences (13.1) give rise to long exact sequences when we apply $\operatorname{Ext}_{H_{*} H}(-)$. Therefore, upon applying Ext to the $E$-Adams resolution, we get a spectral sequence

$$
E_{1}^{s, t, k}\left(H_{*} X\right)=\operatorname{Ext}^{s-k, t}\left(H_{*} W_{k}^{k}(X)\right) \Rightarrow \operatorname{Ext}^{s, t}\left(H_{*} X\right)
$$

called the Mahowald spectral sequence (MSS). For the remainder of this section we shall assume $H=H \mathbb{F}_{p}$.

Many of the notions that we defined for the homotopy root invariant carry over to the algebraic context. We may define Tate comodules (over the dual Steenrod algebra)

$$
t\left(H_{*} X\right)={\underset{\lim }{N}}_{N}\left(H_{*}\left(X \wedge \Sigma P_{-N}\right)\right)=(t H \wedge X)_{*} \cdot
$$

Here, it is essential that the limit is taken after taking homology. We may use this to define algebraic $E$-root invariants.

Definition 13.1 (Algebraic E-root invariant). Let $\alpha$ be an element of the Ext group $\operatorname{Ext}^{s, t}\left(H_{*} X\right)$. We have the following diagram of Ext groups which defines the algebraic $E$-root invariant $R_{E, \text { alg }}(\alpha)$ :

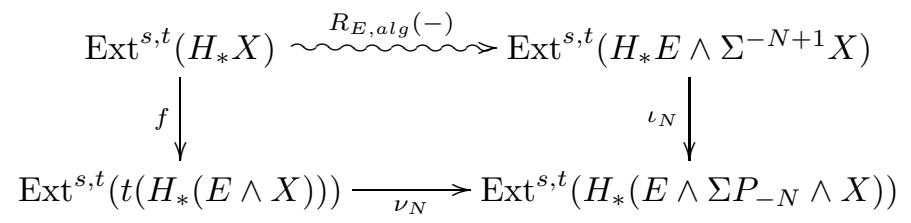

Here $f$ is induced by the inclusion of the 0 -cell of $t S^{0}, \nu_{N}$ is the projection onto the $-N$-coskeleton, $\iota_{N}$ is inclusion of the $-N$-cell, and $N$ is minimal with respect to the property that $\nu_{N} \circ f(\alpha)$ is non-zero. Then the algebraic $E$-root invariant $R_{E, \text { alg }}(\alpha)$ is defined to be the coset of lifts $\gamma \in \operatorname{Ext}^{s, t+N-1}\left(H_{*} E \wedge X\right)$ of the element $\nu_{N} \circ f(\alpha)$. It could be the case that $f(\alpha)=0$, in which case we say that the algebraic $E$-root invariant is trivial.

For simplicity, we now restrict our attention to the case $E=B P$, and we work at an odd prime $p$. Let $\bar{\Lambda}$ be the periodic lambda algebra 14. The cohomology of $\bar{\Lambda}$ is $\operatorname{Ext}\left(H_{*}\right)$. The MSS has a concrete description in terms of $\bar{\Lambda}$. Define a decreasing filtration on $\bar{\Lambda}$ by

$$
\bar{\Lambda}=F_{0} \bar{\Lambda} \subset F_{1} \bar{\Lambda} \subset F_{2} \bar{\Lambda} \subset \cdots,
$$

where $F_{k} \bar{\Lambda}$ is the subcomplex of $\bar{\Lambda}$ generated by monomials containing $k$ or more $\lambda_{i}$ 's. The spectral sequence associated to the filtered complex $\left\{F_{k} \bar{\Lambda}\right\}$ is isomorphic to the MSS starting with the $E_{2}$-term. The spectral sequence of the filtered complex would agree with the MSS on the level of $E_{1}$-terms if one were to apply $\operatorname{Ext}\left(H_{*}-\right)$ to 
the correct $B P$-resolution (which differs from the canonical $B P$-resolution). Therefore, we shall also refer to the spectral sequence associated to the filtered complex $\left\{F_{k} \bar{\Lambda}\right\}$ as an MSS.

In analogy with the spaces $W_{s}(X)$, define subcomplexes of $\bar{\Lambda} \otimes H_{*}(X)$ (the complex which computes $\left.\operatorname{Ext}\left(H_{*} X\right)\right)$ by

$$
W_{k}\left(H_{*} X\right)=F_{k} \bar{\Lambda} \otimes H_{*}(X) .
$$

We define quotients $W_{k}^{l}\left(H_{*} X\right)$ by

$$
W_{k}^{l}\left(H_{*} X\right)=W_{k}\left(H_{*} X\right) / W_{l+1}\left(H_{*} X\right) .
$$

The cohomology $H^{*}\left(W_{k}^{l}\left(H_{*} X\right)\right)$ is computed by restricting the MSS by setting the $E_{1}^{*, *, i}$-term equal to zero for $i<k$ or $i>l$. We define complexes $W_{k}^{l}\left(H_{*} P^{N}\right)$ to be the inverse limit

$$
W_{k}^{l}\left(H_{*} P^{N}\right)=\lim _{M} W_{k}^{l}\left(H_{*} P_{-M}^{N}\right)
$$

For sequences

$$
\begin{aligned}
& I=\left\{k_{1}<k_{2}<\cdots<k_{l}\right\}, \\
& J=\left\{N_{1}<N_{2}<\cdots<N_{l}\right\}
\end{aligned}
$$

we can define filtered Tate complexes

$$
W_{I}\left(H_{*} P^{J} \wedge X\right)=\sum_{i} W_{k_{i}}\left(H_{*} P^{N_{i}} \wedge X\right) .
$$

Given another pair of sequences $\left(I^{\prime}, J^{\prime}\right) \leq(I, J)$, we define complexes

$$
W_{I}^{I^{\prime}}\left(H_{*} P_{J^{\prime}}^{J} \wedge X\right)=W_{I}\left(H_{*} P^{J} \wedge X\right) / W_{I^{\prime}+1}\left(H_{*} P^{J^{\prime}-1} \wedge X\right)
$$

where $I^{\prime}+1$ (respectively $J^{\prime}-1$ ) is the sequence obtained by increasing (decreasing) every element of the sequence by 1 .

We shall now define algebraic filtered root invariants in analogy with the definition of filtered root invariants given in Section 3. Let $\alpha$ be an element of $\operatorname{Ext}^{s, t}\left(H_{*} X\right)$. We shall describe a pair of sequences

$$
\begin{aligned}
& I=\left\{k_{1}<k_{2}<\cdots<k_{l}\right\}, \\
& J=\left\{-N_{1}<-N_{2}<\cdots<-N_{l}\right\}
\end{aligned}
$$

associated to $\alpha$, which we define inductively. Let $k_{1} \geq 0$ be maximal such that the image of $\alpha$ under the composite

$$
\mathrm{Ext}^{s, t-1}\left(H_{*} \Sigma^{-1} X\right) \rightarrow H^{s, t-1}\left(W_{0}^{k_{1}-1}\left(H_{*} P \wedge X\right)_{-\infty}\right)
$$

is trivial. Next, choose $N_{1}$ to be maximal such that the image of $\alpha$ under the composite

$$
\operatorname{Ext}^{s, t-1}\left(H_{*} \Sigma^{-1} X\right) \rightarrow H^{s, t-1}\left(W_{0}^{\left(k_{1}-1, k_{1}\right)}\left(H_{*} P_{\left(-N_{1}+1, \infty\right)} \wedge X\right)\right)
$$

is trivial. Inductively, given

$$
\begin{aligned}
I^{\prime} & =\left(k_{1}, k_{2}, \ldots, k_{i}\right), \\
J^{\prime} & =\left(-N_{1},-N_{2}, \ldots,-N_{i}\right),
\end{aligned}
$$

let $k_{i+1}$ be maximal so that the image of $\alpha$ under the composite

$$
\operatorname{Ext}^{s, t-1}\left(H_{*} \Sigma^{-1} X\right) \rightarrow H^{s, t-1}\left(W_{0}^{\left(I^{\prime}-1, k_{i+1}-1\right)}\left(H_{*} P_{\left(J^{\prime}+1, \infty\right)} \wedge X\right)\right)
$$


is trivial. If there is no such maximal $k_{i+1}$, we declare that $k_{i+1}=\infty$ and we are finished. Otherwise, choose $N_{i+1}$ to be maximal such that the composite

$$
\mathrm{Ext}^{s, t-1}\left(H_{*} \Sigma^{-1} X\right) \rightarrow H^{s, t-1}\left(W_{0}^{\left(I^{\prime}-1, k_{i+1}-1, k_{i+1}\right)}\left(H_{*} P_{\left(J^{\prime}+1,-N_{i+1}+1, \infty\right)} \wedge X\right)\right)
$$

is trivial, and continue the inductive procedure. We shall refer to the pair $(I, J)$ as the $B P$-bifiltration of $\alpha$.

Observe that there is an exact sequence

$H^{s, t-1}\left(W_{I}\left(H_{*} P^{J} \wedge X\right)\right) \rightarrow \mathrm{Ext}^{s, t-1}\left(t\left(H_{*} \Sigma^{-1} X\right)\right) \rightarrow H^{s, t-1}\left(W^{I-1}\left(H_{*} P_{J+1} \wedge X\right)\right)$.

Our choice of $(I, J)$ ensures that the image of $\alpha$ in $H^{s, t-1}\left(W^{I-1}\left(H_{*} P_{J+1} \wedge X\right)\right)$ is trivial. Thus $\alpha$ lifts to an element $f^{\alpha} \in H^{s, t-1}\left(W_{I}\left(H_{*} P^{J} \wedge X\right)\right)$.

Definition 13.2 (Algebraic filtered root invariants). Let $X$ be a finite complex and let $\alpha$ be an element of $\operatorname{Ext}^{s, t}\left(H_{*} X\right)$ of $B P$-bifiltration $(I, J)$. Given a lift $f^{\alpha} \in H^{s, t-1}\left(W_{I}\left(H_{*} P^{J} \wedge X\right)\right)$, the $k^{\text {th }}$ algebraic filtered root invariant is said to be trivial if $k \neq k_{i}$ for any $k_{i} \in I$. Otherwise, if $k=k_{i}$ for some $i$, we say that the image $\beta$ of $f^{\alpha}$ under the quotient map

$$
H^{s, t-1}\left(W_{I}\left(H_{*} P^{J} \wedge X\right)\right) \rightarrow H^{s, t-1}\left(W_{k_{i}}^{k_{i}}\left(H_{*} \Sigma^{-N_{i}} X\right)\right)
$$

is an element of the $k^{\text {th }}$ algebraic filtered root invariant of $\alpha$. The $k^{\text {th }}$ algebraic filtered root invariant is the coset $R_{E, a l g}^{[k]}(\alpha)$ of the MSS $E_{1}$-term $E_{1}^{s, t+N_{i}-1, k}\left(H_{*} X\right)$ of all such $\beta$ as we vary the lift $f^{\alpha}$.

We wish to indicate how our filtered root invariant theorems carry over to the algebraic context. In order to do this we must produce algebraic versions of $K$ Toda brackets. Suppose that $M$ is a finite $A_{*}$-comodule concentrated in degrees 0 through $n$ with a single $\mathbb{F}_{p}$ generator in degrees 0 and $n$. In what follows, we let $M^{j}$ be the sub-comodule of $M$ consisting of elements of degree less than or equal to $j$, and let $M_{i}^{j}$ be the quotient $M^{j} / M^{i-1}$. We shall omit the top index for the quotient $M_{i}=M / M^{i-1}$.

Definition 13.3 (Algebraic $M$-Toda bracket). Let

$$
f: \operatorname{Ext}^{s, t}\left(M_{1}\right) \rightarrow \operatorname{Ext}^{s+1, t}\left(\mathbb{F}_{p}\right)
$$

be the connecting homomorphism associated to the short exact sequence

$$
0 \rightarrow \mathbb{F}_{p} \rightarrow M \rightarrow M_{1} \rightarrow 0 .
$$

Let $\nu: M_{1} \rightarrow \Sigma^{n} \mathbb{F}_{p}$ be the projection onto the top generator. Suppose $\alpha$ is an element of $\mathrm{Ext}^{s, t}\left(H_{*} X\right)$. We have

$$
\operatorname{Ext}^{s, t}\left(H_{*} X\right) \stackrel{\nu_{*}}{\longleftarrow} \mathrm{Ext}^{s, t+n}\left(H_{*} X \otimes M_{1}\right) \stackrel{f}{\longrightarrow} \operatorname{Ext}^{s+1, t+n}\left(H_{*} X\right) .
$$

We say the algebraic $M$-Toda bracket

$$
\langle M\rangle(\alpha) \subseteq \operatorname{Ext}^{s+1, t+n}\left(H_{*} X\right)
$$

is defined if $\alpha$ is in the image of $\nu_{*}$. Then the algebraic $M$-Toda bracket is the collection of all $f(\gamma) \in \mathrm{Ext}^{s+1, t+n}\left(H_{*} X\right)$, where $\gamma \in \mathrm{Ext}^{s, t+n}\left(H_{*} X \otimes M_{1}\right)$ is any element satisfying $\nu_{*}(\gamma)=\alpha$.

We leave it to the reader to construct the dually defined algebraic $M$-Toda bracket analogous to the dually defined $K$-Toda bracket, and MSS version of the algebraic $M$-Toda bracket analogous to the $E$-ASS version of the $K$-Toda bracket. 
The definitions of these Toda brackets given in Section 4 carry over verbatim to the algebraic context.

With these definitions, our filtered root invariant results given in Section 5 have analogous algebraic statements. The first algebraic filtered root invariant is the algebraic $B P$-root invariant. If the $k^{\text {th }}$ algebraic filtered root invariant is a permanent cycle in the MSS, then it is the algebraic root invariant modulo $B P$-filtration greater than $k$. One may deduce higher algebraic filtered root invariants from differentials and compositions in the MSS. In other words, there are algebraic versions of Theorem 5.1, Corollary 5.2, Theorem 5.3, Theorem 5.4, Proposition 5.7. Proposition 5.8, and Corollary 5.9, where one makes the following replacements:

- Replace $\pi_{*}$ with Ext.

- Replace root invariants with algebraic root invariants.

- Replace $E$-root invariants with algebraic $E$-root invariants.

- Replace filtered root invariants with algebraic filtered root invariants.

- Replace $K$-Toda brackets with algebraic $H_{*}(K)$-Toda brackets.

- Specialize from $E$ to $B P$.

- Replace the E-ASS with the MSS.

\section{MODIFIED FILTERED ROOT INVARIANTS}

In this section we will recall the modified root invariant that Mahowald and Ravenel define in [25]. We will then explain how to define modified versions of filtered root invariants, and how to adapt our theorems to compute modified root invariants. We also discuss algebraic modified filtered root invariants, which is the tool we will be using in Section 15 .

Let $X$ be a finite complex. We shall begin be recalling the definition of the modified root invariant $R^{\prime}(\alpha)$ for an element $\alpha \in \pi_{t}(X)$. Let $V(0)$ be the $\bmod$ $p$ Moore spectrum. We define modified versions of the stunted Tate spectra $P_{M}^{N}$ which will have the same underlying space, but which we shall view as being built out of $V(0)$ instead of the sphere spectrum. To this end, define spectra as

$$
P^{\prime N}= \begin{cases}P^{N+1} & \text { if } N \text { is of the form } k q-1, \\ P^{N} & \text { otherwise }\end{cases}
$$

Likewise, for $M \leq N$, define quotient spectra as

$$
P_{M}^{\prime N}=P^{\prime N} / P^{\prime M-1} \text {. }
$$

Thus the spectrum ${P^{\prime}}_{M}^{N}$ has a $V(0)$-cell in every dimension from $M$ to $N$ congruent to -1 modulo $q$. There are cofiber sequences

$$
{P^{\prime}}_{k q-1}^{(l-1) q-1} \rightarrow{P^{\prime}}_{k q-1}^{l q-1} \rightarrow \Sigma^{l q-1} V(0) .
$$

Definition 14.1 (Modified root invariant). Let $X$ be a finite complex, and suppose we are given $\alpha \in \pi_{t}(X)$. The modified root invariant of $\alpha$ is the coset of all dotted 
arrows making the following diagram commute:

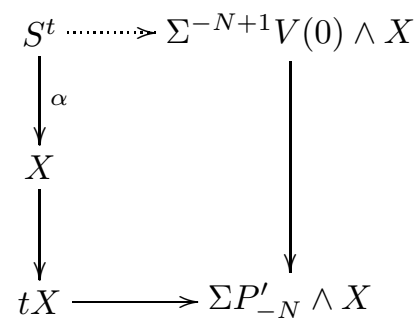

This coset is denoted $R^{\prime}(\alpha)$. Here $N$ is chosen to be minimal such that the composite $S^{t} \rightarrow \Sigma P_{-N}^{\prime} \wedge X$ is non-trivial.

Thus the definition of the modified root invariant differs from the definition of the root invariant in that it takes values in $\pi_{*}(V(0) \wedge X)$ instead of $\pi_{*}(X)$, and that $P$ has been replaced with $P^{\prime}$. By making these replacements elsewhere, we may produce modified versions of our other definitions, as summarized below. In the case of the algebraic filtered root invariants, we choose to use the quotient $\bar{\Lambda}_{(0)}=\bar{\Lambda} /\left(v_{0}\right)$ described in [14. The cohomology of $\bar{\Lambda}_{(0)}$ is given by

$$
H^{*}\left(\bar{\Lambda}_{(0)}\right)=\operatorname{Ext}\left(H_{*} V(0)\right) \text {. }
$$

One has modified versions of the subcomplexes $W_{k}\left(H_{*} X\right)$ given by

$$
W_{k}^{\prime}\left(H_{*} X\right)=\operatorname{Im}\left(W_{k}\left(H_{*} X\right) \hookrightarrow \bar{\Lambda} \otimes H_{*}(X) \rightarrow \bar{\Lambda}_{(0)} \otimes H_{*}(X)\right) .
$$

By modifying our definitions, we produce:

- Modified E-root invariants

$$
R_{E}^{\prime}: \pi_{*}(X) \rightsquigarrow \pi_{*}(E \wedge V(0) \wedge X) .
$$

- Modified algebraic root invariants

$$
R_{a l g}^{\prime}: \operatorname{Ext}\left(H_{*} X\right) \rightsquigarrow \operatorname{Ext}\left(H_{*} V(0) \wedge X\right) .
$$

- Modified filtered Tate spectra

$$
W_{I}\left(P^{\prime J}\right) .
$$

- Modified filtered root invariants

$$
R_{E}^{[k]^{\prime}}: \pi_{*}(X) \rightsquigarrow \pi_{*}\left(W_{k}^{k}(V(0) \wedge X)\right) .
$$

- Modified algebraic E-root invariants

$$
R_{E, \text { alg }}^{\prime}: \operatorname{Ext}\left(H_{*} X\right) \rightsquigarrow \operatorname{Ext}\left(H_{*} E \wedge V(0) \wedge X\right) .
$$

- Modified algebraic filtered root invariants

$$
R_{E, a l g}^{[k]^{\prime}}: \operatorname{Ext}\left(H_{*} X\right) \rightsquigarrow H^{*}\left({W^{\prime}}_{k}^{k}\left(H_{*} X\right)\right) .
$$

We would like to reformulate the results of Section 5 in terms of modified root invariants. We need a modified version of the Toda brackets which appear in the statements of the main theorems. Suppose that $K$ is a finite complex built out of $V(0)$ with a single bottom $V(0)$-cell in dimension 0 and a single top $V(0)$-cell in dimension $n$. Let $K^{j}$ be the $j^{\text {th }} V(0)$-skeleton of $K$, and let $K_{i}^{j}$ be the quotient $K^{j} / K^{i-1}$. We shall omit the top index for the $i^{\text {th }} V(0)$-coskeleton $K_{i}=K / K^{i-1}$. 
Definition 14.2 (Modified $K$-Toda bracket). Let

$$
f: \Sigma^{-1} K_{1} \rightarrow V(0)
$$

be the attaching map of the first $V(0)$-coskeleton of $K$ to the zeroth $V(0)$-cell, so that the cofiber of $f$ is $K$. Let $\nu: K_{1} \rightarrow \Sigma^{n} V(0)$ be the projection onto the top $V(0)$-cell. Suppose $\alpha$ is an element of $\pi_{t}(X \wedge V(0))$. We have

$$
\pi_{t}(X \wedge V(0)) \stackrel{\nu_{*}}{\longleftarrow} \pi_{t+n}\left(X \wedge K_{1}\right) \stackrel{f_{*}}{\longrightarrow} \pi_{t+n-1}(X \wedge V(0)) .
$$

We say the modified $K$-Toda bracket

$$
\langle K\rangle^{\prime}(\alpha) \subseteq \pi_{t+n-1}(X \wedge V(0))
$$

is defined if $\alpha$ is in the image of $\nu_{*}$. Then the modified $K$-Toda bracket is the collection of all $f_{*}(\gamma) \in \pi_{t+n-1}(X \wedge V(0))$, where $\gamma \in \pi_{t+n}\left(X \wedge K_{1}\right)$ is any element satisfying $\nu_{*}(\gamma)=\alpha$.

Similarly we may provide a modified version of the dually defined and E-ASS $K$-Toda brackets given in Section 4. Furthermore, for an $A_{*}$-comodule $M$ which is cofree over the coalgebra $E\left[\tau_{0}\right]$, it is straightforward to define the modified algebraic $M$-Toda bracket by varying the definition of the $M$-Toda bracket given in Section 13 .

Modified versions of all of the results in Section 5 hold, with proofs that go through with only superficial changes. Specifically, one must make the following adjustments:

- Replace $P$ with $P^{\prime}$.

- Replace root invariants with modified root invariants.

- Replace $E$-root invariants with modified $E$-root invariants.

- Replace filtered root invariants with modified filtered root invariants.

- Replace $K$-Toda brackets with modified $K$-Toda brackets.

- Replace the $E$-ASS for $X$ with the $E$-ASS for $X \wedge V(0)$.

Furthermore, there are algebraic modified versions of Theorems 5.1, 5.3 and 5.4 Propositions 5.7 and 5.8 , and Corollaries 5.2 and 5.9, where one makes the following replacements:

- Replace $P$ with $P^{\prime}$.

- Replace $\pi_{*}$ with Ext.

- Replace root invariants with modified algebraic root invariants.

- Replace $E$-root invariants with modified algebraic $E$-root invariants.

- Replace filtered root invariants with modified algebraic filtered root invariants.

- Replace $K$-Toda brackets with modified algebraic $H_{*}(K)$-Toda brackets.

- Specialize from $E$ to $B P$.

- Replace the $E$-ASS for $X$ with the MSS for $H_{*} X \wedge V(0)$.

We now modify our definitions even further, to give variations of the second modified filtered root invariant $R^{\prime \prime}(\alpha)$ given in [25. In what follows we shall always be working at a prime $p \geq 3$.

It is observed in 25] that there is a splitting

$$
P_{k q-1}^{(l+1) q} \wedge V(0) \simeq P_{k q-1}^{\prime \prime l q-1} \vee \Sigma^{k q} V(0) \vee \Sigma^{(l+1) q-1} V(0) .
$$


In the notation of [25], we have

$$
P_{k q-1}^{\prime \prime l q-1}=\bar{P}_{k q-1}^{l q}
$$

The spectra $P_{k q-1}^{\prime \prime l q-1}$ are built out of the Smith-Toda complex $V(1)$. There is a $V(1)$-cell every in dimension from $k q-1$ to $l q-1$ congruent to $-1 \bmod q$. The decomposition of $P_{-\infty}^{\prime \prime}$ into $V(1)$-cells is described on the level of cohomology in the following lemma, which is a straightforward computation.

Lemma 14.3. Let $e_{n}^{*}$ be the generator of $H^{*}(P)_{-\infty}$ in dimension $n$, where $n \equiv$ $0,-1(\bmod q)$. The cohomology of the Moore spectrum is given by

$$
H^{*}(V(0))=E\left[Q_{0}\right]
$$

as a module over the Steenrod algebra. The cohomology

$$
H^{*}\left(P^{\prime \prime}\right)_{-\infty}=H^{*}(P \wedge V(0))_{-\infty}
$$

is free over the subalgebra $E\left[Q_{0}, Q_{1}\right]$ of the Steenrod algebra on the generators $e_{k q-1}^{*} \otimes 1$. The action $E\left[Q_{0}, Q_{1}\right]$ on the free $E\left[Q_{0}, Q_{1}\right]$-submodule generated by $e_{k q-1}^{*} \otimes 1$ is then given by the following formulas:

$$
\begin{aligned}
Q_{0}\left(e_{k q-1}^{*} \otimes 1\right) & =e_{k q}^{*} \otimes 1-e_{k q-1}^{*} \otimes Q_{0}, \\
Q_{1}\left(e_{k q-1}^{*} \otimes 1\right) & =e_{(k+1) q}^{*} \otimes 1, \\
Q_{1}\left(e_{k q}^{*} \otimes 1-e_{k q-1}^{*} \otimes Q_{0}\right) & =-e_{(k+1) q}^{*} \otimes Q_{0}, \\
Q_{0}\left(e_{(k+1) q}^{*} \otimes 1\right) & =e_{(k+1) q}^{*} \otimes Q_{0} .
\end{aligned}
$$

It is convenient to allow arbitrary subscripts and superscripts, so we define for integers $M \leq N$

$$
P_{M}^{\prime \prime N}=P_{k q-1}^{\prime \prime l q-1}
$$

where $k$ (respectively $l$ ) is minimal (maximal) such that $M \leq k q-1 \leq N(M \leq$ $l q-1 \leq N)$. If there is no such $k$ and $l$, then we have $P^{\prime \prime}{ }_{M}^{N}=*$.

The following lemma is Lemma 3.7(e) of 25.

Lemma 14.4. If $k$ and $l$ are congruent to $0(\bmod p)$, then $H^{*}\left(P_{k q-1}^{\prime \prime l q-1}\right)$ is free over the subalgebra $A(1)$ of the Steenrod algebra.

Since there is no difference between the spectra $P_{-\infty}^{\prime \prime}$ and $P_{-\infty} \wedge V(0)$, and since $V(0)$ is $p$-complete, there is an analog of Lin's theorem [25, Lemma 3.7(b)].

Lemma 14.5. The map

$$
\Sigma^{-1} V(0) \rightarrow P_{-\infty}^{\prime \prime}
$$

is an equivalence.

In light of Lemma 14.5 Mahowald and Ravenel 25 define a second modified root invariant.

Definition 14.6 (Second modified root invariant). Let $X$ be a finite complex, and suppose we are given $\alpha \in \pi_{t}(X \wedge V(0))$. The second modified root invariant of $\alpha$ is 
the coset of all dotted arrows making the following diagram commute:

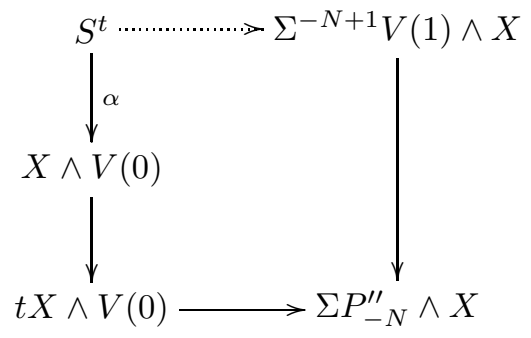

This coset is denoted $R^{\prime \prime}(\alpha)$. Here $N$ is chosen to be minimal such that the composite $S^{t} \rightarrow \Sigma P_{-N}^{\prime \prime} \wedge X$ is non-trivial.

The definition of the second modified root invariant may be obtained from the definition of the (first) modified root invariant by smashing $X$ with $V(0)$, replacing $P^{\prime}$ with $P^{\prime \prime}$, and replacing $V(0)$ with $V(1)$. Similarly, one may obtain second modified versions of all of the other definitions we have been working with, as summarized below. The second modified lambda complex $W_{k}^{\prime \prime}\left(H_{*} X\right)$ is the appropriate subcomplex of $\bar{\Lambda}_{(1)} \otimes H_{*}(X)$, where $\bar{\Lambda}_{(1)}=\bar{\Lambda} /\left(v_{0}, v_{1}\right)$.

- Second modified $E$-root invariants

$$
R_{E}^{\prime \prime}: \pi_{*}(X \wedge V(0)) \rightsquigarrow \pi_{*}(E \wedge V(1) \wedge X) .
$$

- Second modified algebraic root invariants

$$
R_{\text {alg }}^{\prime \prime}: \operatorname{Ext}\left(H_{*} X \wedge V(0)\right) \rightsquigarrow \operatorname{Ext}\left(H_{*} V(1) \wedge X\right) .
$$

- Second modified filtered Tate spectra

$$
W_{I}\left(P^{\prime \prime}\right) \text {. }
$$

- Second modified filtered root invariants

$$
R_{E}^{[k] "}: \pi_{*}(X \wedge V(0)) \rightsquigarrow \pi_{*}\left(W_{k}^{k}(V(1) \wedge X)\right) .
$$

- Second modified algebraic E-root invariants

$$
R_{E, \text { alg }}^{\prime \prime}: \operatorname{Ext}\left(H_{*} X \wedge V(0)\right) \rightsquigarrow \operatorname{Ext}\left(H_{*} E \wedge V(1) \wedge X\right) .
$$

- Second modified algebraic filtered root invariants

$$
R_{E, \text { alg }}^{[k]^{\prime \prime}}: \operatorname{Ext}\left(H_{*} X \wedge V(0)\right) \rightsquigarrow H^{*}\left({W^{\prime \prime}}_{k}^{k}\left(H_{*} X\right)\right) .
$$

If $K$ is a finite complex built from $V(1)$ with single bottom- and top-dimensional $V(1)$-cells, then we may define the second modified $K$-Toda bracket by

$$
\langle K\rangle^{\prime \prime}: \pi_{*}(V(1) \wedge X) \rightsquigarrow \pi_{*}(V(1) \wedge X) .
$$

Likewise, if $M$ is an $A_{*}$-comodule which is cofree over $E\left[\tau_{0}, \tau_{1}\right]$, one can define a second modified algebraic $M$-Toda bracket on $\operatorname{Ext}\left(H_{*} V(1) \wedge X\right)$. Just as outlined for the case of modified root invariants in the first half of this section, second modified and second modified algebraic versions of the results of Section 5 hold. 
15. Computation of Some infinite families of Root invariants at $p=3$

In this section we will extend the computation

$$
\left(-v_{2}\right)^{i} \in R^{\prime \prime}\left(v_{1}^{i}\right)
$$

of 25 . for $p \geq 5$ to the prime 3 for $i \equiv 0,1,5(\bmod 9)$. We will use our modified root invariant computations to deduce that, at the prime 3 , the root invariant of the element $\alpha_{i} \in \pi_{*}^{S}$ is given by the element $\beta_{i}$ for $i \equiv 0,1,5(\bmod 9)$.

Throughout this section we work at the prime 3 . Low-dimensional computations indicate that there is a map

$$
v_{2}: S^{16} \rightarrow V(1)
$$

Oka [31] demonstrates that there is a map

$$
v_{2}^{5}: S^{80} \rightarrow V(1)
$$

Composing these maps with iterates of the map

$$
v_{2}^{9}: \Sigma^{144} V(1) \rightarrow V(1)
$$

given in [4, we have maps

$$
v_{2}^{i}: S^{16 i} \rightarrow V(1)
$$

for $i \cong 0,1,5(\bmod 9)$. The computation of $\pi_{*}\left(L_{2} V(1)\right)$ given in 12 indicates that these are the only $i$ for which $v_{2}^{i}$ can exist.

Theorem 15.1. At the prime 3 , for $i \equiv 0,1,5(\bmod 9)$, the second modified root invariant of $v_{1}^{i} \in \pi_{*}(V(0))$ is given by

$$
\left(-v_{2}\right)^{i} \in R^{\prime \prime}\left(v_{1}^{i}\right) .
$$

We shall prove Theorem 15.1 with a sequence of lemmas.

Lemma 15.2. For all $i$, we have the second modified BP-root invariant

$$
\left(-v_{2}\right)^{i} \in R_{B P}^{\prime \prime}\left(v_{1}^{i}\right) .
$$

Proof. Modulo $I_{1}$, the 3 -series of the formal group associated to $B P$ is given by

$$
[3](x) \equiv v_{1} x^{3}+v_{2} x^{9}+\cdots \quad\left(\bmod I_{1}\right) .
$$

Thus in $t B P \wedge V(0)_{*}$ (see Lemma 10.4), we have

$$
v_{1}=-v_{2} x^{6}+\cdots
$$

and upon taking the $i^{\text {th }}$ power, we have

$$
\left(v_{1}\right)^{i}=\left(-v_{2}\right)^{i} x^{6 i}+\cdots .
$$

Using the second modified version of Corollary 10.5, we may deduce the result.

Lemma 15.3. For all $i$, we have the second modified algebraic BP-root invariant

$$
\left(-v_{2}\right)^{i} \in R_{B P, \text { alg }}^{\prime \prime}\left(v_{1}^{i}\right) .
$$

Here, $v_{1}$ and $v_{2}$ are viewed as elements in the cohomology of the periodic lambda algebra.

Proof. Apply $\operatorname{Ext}\left(H_{*}-\right)$ to the diagram which realizes the second modified $B P$-root invariant given by Lemma 15.2

In order to eliminate obstructions in higher Adams filtration, we prove the following lemma. This lemma is essentially contained in the proof of Lemma 3.10 of [25]. 
Lemma 15.4. We have the following Ext calculation:

$$
\mathrm{Ext}^{s, i q-1+s}\left(H_{*} P_{-3 i q-1}^{\prime \prime}\right)= \begin{cases}0, & s>i, \\ \mathbb{F}_{p}\left\{v_{2}^{i}\right\}, & s=i .\end{cases}
$$

Proof. In Lemma 14.4 we saw that $H^{*}\left(P_{-3 i q-1}^{\prime \prime}\right)$ is free over the subalgebra $A(1)$ of the Steenrod algebra on generators in dimensions congruent to $-1(\bmod 3 q)$. Therefore $\operatorname{Ext}_{A_{*}}\left(H_{*} P_{-3 i q-1}^{\prime \prime}\right)$ is built out of $\operatorname{Ext}_{A_{*}}\left(\Sigma^{3 k q-1} A(1)_{*}\right)$ for $k \geq-i$. Now the May spectral sequence $E_{2}$-term for $\operatorname{Ext}_{A_{*}}\left(A(1)_{*}\right)$ is given by

$E_{2}=E\left[h_{i, j}: i \geq 1, j \geq 0, i+j \geq 2\right] \otimes P\left[b_{i, j}: i \geq 1, j \geq 0, i+j \geq 2\right] \otimes P\left[v_{i}: i \geq 2\right]$.

Analyzing the degrees that these elements live in establishes that the only elements in $\operatorname{Ext}_{A_{*}}\left(A(1)_{*}\right)$ which lie on or above the line of slope $1 /\left|v_{2}\right|$ (in the $(t-s, s)$-plane) are those of the form $h_{1,1}^{\epsilon_{1}} h_{2,0}^{\epsilon_{2}} v_{2}^{k}$ for $\epsilon_{j}=0,1$. Therefore, one easily deduces that there are no elements which lie above the line of slope $1 /\left|v_{2}\right|$ passing through the point $(0,-3 i q-1)$ which lie in the dimensions we are considering, and the only element which lies in the $i q-1$ stem with Adams filtration equal to $i$ is $v_{2}^{i}$.

Lemma 15.5. For all $i$, we have the second modified algebraic root invariant

$$
\left(-v_{2}\right)^{i} \in R_{\text {alg }}^{\prime \prime}\left(v_{1}^{i}\right) .
$$

Proof. Applying the second modified algebraic version of Proposition 5.7 we may deduce that the zeroth second modified algebraic filtered root invariant is given by

$$
\left(-v_{2}^{i}\right) \in R_{B P, \text { alg }}^{[0]}{ }^{\prime \prime}\left(v_{1}^{i}\right) .
$$

The element $v_{2}^{i}$ is a permanent cycle in the MSS for $\operatorname{Ext}\left(H_{*} V(1)\right)$, so we may use the second modified algebraic version of Theorem 5.1 to deduce that the difference of the images of $\left(-v_{2}\right)^{i}$ and $v_{1}^{i}$ in

$$
\operatorname{Ext}^{i, i q-1+i}\left(H_{*} P_{-3 i q-1}^{\prime \prime}\right)
$$

is of $B P$-filtration greater than 0. Lemma 15.4 says that there are no non-zero elements of this Ext group which could represent the difference of these images, so we actually have

$$
\left(-v_{2}\right)^{i} \in R_{a l g}^{\prime \prime}\left(v_{1}^{i}\right)
$$

We shall need a slightly different result for $i \equiv 5(\bmod 9)$.

Lemma 15.6. Modulo indeterminacy, the second modified algebraic root invariant of $v_{1}^{9 t+5}$ is also given by

$$
\left(-v_{2}\right)^{9 t+5} \pm v_{2}^{9 t} v_{3} g_{0} b_{0} \in R_{\text {alg }}^{\prime \prime}\left(v_{1}^{9 t+5}\right) .
$$

Proof. By Lemma 15.4 the element $v_{2}^{9 t} v_{3} g_{0} b_{0}$ is in the kernel of the map

$$
\operatorname{Ext}^{i, i q-1+i}\left(H_{*} \Sigma^{-3 i q-1} V(1)\right) \rightarrow \mathrm{Ext}^{i, i q-1+i}\left(H_{*} P_{-3 i q-1}^{\prime \prime}\right),
$$

where $i=9 t+5$. Therefore it is in the indeterminacy of the second modified algebraic root invariant.

Proof of Theorem 15.1. The element $v_{2}^{i}$ is a permanent cycle in the ASS for $V(1)$ for $i=0,1$. The existence of the self map $v_{2}^{9}$ of the complex $V(1)$ implies that $v_{2}^{i}$ is a permanent cycle in the ASS for $i \equiv 0,1(\bmod 9)$. However, in the ASS for $V(1)$, the element $v_{2}^{5}$ is not a permanent cycle; the element

$$
v_{2}^{5} \pm v_{3} g_{0} b_{0}
$$


is the permanent cycle that detects the Oka element $v_{2}^{5} \in \pi_{80}(V(1))$ [4, Remark 8.2]. Thus the element $v_{2}^{9 t+5} \in \pi_{*}(V(1))$ is detected in the ASS by the permanent cycle $v_{2}^{9 t+5} \pm v_{2}^{9 t} v_{3} g_{0} b_{0}$. For uniformity of notation, let $\widetilde{v_{2}^{i}}$ denote the ASS element that detects $v_{2}^{i} \in \pi_{*}(V(1))$ for $i \equiv 0,1,5(\bmod 9)$.

Lemmas 15.5 and 15.6 imply that we have second modified algebraic root invariants

$$
(-1)^{i} \tilde{v_{2}^{i}} \in R_{a l g}^{\prime \prime}\left(v_{1}^{i}\right)
$$

where $i \equiv 0,1,5(\bmod 9)$. By the second modified version of Theorem 5.10 , we have second modified filtered root invariants given by

$$
(-1)^{i} \widetilde{v_{2}^{i}} \in R_{H}^{[i]^{\prime \prime}}\left(v_{1}^{i}\right)
$$

The elements $\widetilde{v_{2}^{i}}$ are permanent cycles in the ASS, so by the second modified version of Theorem 5.1, we may conclude that the difference between the images of $\left(-v_{2}\right)^{i}$ and $v_{1}^{i}$ in $\pi_{i q-1}\left(P_{-3 i q-1}^{\prime \prime}\right)$ has Adams filtration greater than $i$. According to Lemma 15.4, there are no such elements, so the images of $\left(-v_{2}\right)^{i}$ and $v_{1}^{i}$ in $\pi_{i q-1}\left(P_{-3 i q-1}^{\prime \prime}\right)$ are actually equal, and we have

$$
\left(-v_{2}\right)^{i} \in R^{\prime \prime}\left(v_{1}^{i}\right) \text {. }
$$

We will now use our second modified root invariant computations to deduce the following.

Theorem 15.7. We have root invariants

$$
(-1)^{i+1} \beta_{i} \in R\left(\alpha_{i}\right)
$$

for $i \equiv 0,1,5(\bmod 9)$ at $p=3$.

Proof. Let $\nu$ denote the map which is given by projection onto the top cell of $V(0)$. Since $P_{j q-1}^{\prime \prime}$ is a summand of $P_{j q-1} \wedge V(0)$, we have an induced map

$$
\nu^{\prime}: P_{j q-1}^{\prime \prime} \rightarrow \Sigma P_{(j+1) q} .
$$

We have the following diagram for $i \equiv 0,1,5(\bmod 9)$ :

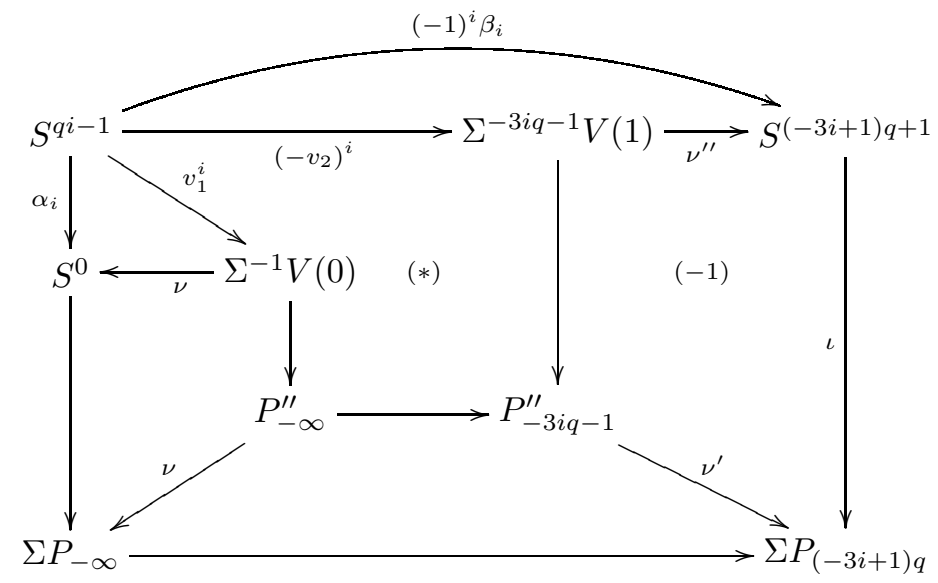

The commutivity of the inner portion $(*)$ is our modified root invariant computations of $v_{1}^{i}$. The portion of the diagram marked with " $(-1)$ " commutes with the 
introduction of a minus sign. This is due to the fact that the elements $\beta_{i}$ are given by $\nu_{*}^{\prime \prime}\left(v_{2}^{i}\right)$, where

$$
\nu^{\prime \prime}: V(1) \rightarrow S^{6}
$$

is the projection onto the cell corresponding to the element $Q_{1} Q_{0}$ in the cohomology group

$$
H^{*}(V(1))=E\left[Q_{0}, Q_{1}\right] .
$$

According to the computations of Lemma 14.3, this is the negative of the cell in $P_{-i 3 q-1}^{\prime \prime}$ which corresponds to the cohomology element

$$
e_{(-3 i+1) q}^{*} \otimes Q_{0}=Q_{0} Q_{1}\left(e_{-3 i q-1}^{*} \otimes 1\right) .
$$

Following the outer portion of the diagram, and introducing the minus sign, we see that we have

$$
(-1)^{i+1} \beta_{i} \in R\left(\alpha_{i}\right)
$$

unless $\iota_{*}\left(\beta_{i}\right)=0$. That is not possible by Proposition 8.7 .

Remark 15.8. If one believes that the root invariant takes $v_{1}^{9}$ multiplication to $v_{2}^{9}$ multiplication at $p=3$, analogous to the results of Sadofsky [35] and Johnson [18, then it is seems likely that $\beta_{i} \dot{\in} R\left(\alpha_{i}\right)$ for $i \equiv 0,1,3,5,6(\bmod 9)$. The present methods will not extend because $v_{2}^{i}$ is not a permanent cycle in the ANSS for $V(1)$ for $i \not \equiv 0,1,5(\bmod 9)$. In fact, at the time of this writing we still do not know that the elements $\beta_{9 t+3}$ exist. The elements $\beta_{9 t+1} \beta_{1} \alpha_{1}$ and $\beta_{9 t+1} \beta_{1}^{4}$ do exist, and are non-trivial by calculations of Shimomura and Wang 34. We conjecture that we have root invariants

$$
\begin{array}{r}
\beta_{9 t+1} \beta_{1} \alpha_{1} \dot{\in} R\left(\alpha_{9 t+2}\right), \\
\beta_{9 t+1} \beta_{1}^{4} \dot{\epsilon} R\left(\alpha_{9 t+4}\right) .
\end{array}
$$

The case $t=0$ was already handled in Section 12

\section{REFERENCES}

[1] J.F. Adams, Stable Homotopy and Generalised Homology, Chicago Lectures in Mathematics, Univ. of Chicago Press, Chicago, IL, 1995. MR1324104 (96a:55002)

[2] M. Ando, J. Morava, H Sadofsky, Completions of $\mathbb{Z} /(p)$-Tate cohomology of periodic spectra, Geometry and Topology 2 (1998) 145-174. MR1638030(99e:55016)

[3] J.F. Adams, J.H. Gunawardena, H. Miller, The Segal conjecture for elementary abelian $p$ groups, Topology 24 (1985) 435-460. MR0816524 (87m:55026)

[4] M. Behrens, S. Pemmaraju, On the existence of the self map $v_{2}^{9}$ on the Smith-Toda complex $V(1)$ at the prime 3, Homotopy theory: relations with algebraic geometry, group cohomology, and algebraic K-theory (Evanston, IL, 2002), Contemp. Math. 346 (2004) 9-49, Amer. Math. Soc., Providence, RI. MR 2066495

[5] R. Bruner, Some remarks on the root invariant, Stable and unstable homotopy (Toronto, ON, 1996), Fields Inst. Commun. 19 (1998) 31-37. MR.1622335 (99b:55023)

[6] R. Bruner, Some root invariants and Steenrod operations in $\operatorname{Ext}_{A}\left(\mathbb{F}_{2}, \mathbb{F}_{2}\right)$, Homotopy theory via algebraic geometry and group representations (Evanston, IL, 1997), Contemp. Math. 220 (1998) 27-33. MR1642887 (99g:55017)

[7] R. Bruner, J. Greenlees, The Bredon-Löffler conjecture, Experiment. Math. 4 (1995) 289-297. MR1387694 (97b:55017)

[8] R.R. Bruner, J.P. May, M. Steinberger, J. McClure, $H_{\infty}$ Ring Spectra and their Applications, Lecture Notes Math., Vol. 1176, Springer, Berlin, 1986. MR0836132 (88e:55001) 
[9] J. Caruso, J.P. May, S.B. Priddy, The Segal conjecture for elementary abelian $p$-groups II, Topology 26 (1987) 413-433. MR0919728 (90c:55005)

[10] D.M. Davis, D.C. Johnson, J. Klippenstein, M. Mahowald, S. Wegmann, The spectrum $(P \wedge \mathrm{BP}\langle 2\rangle)_{-\infty}$, Trans. Amer. Math. Soc. 296 (1986) 95-110. MR0837800 (88b:55011)

[11] D.M. Davis, M. Mahowald, The spectrum $(P \wedge b o)_{-\infty}$, Math. Proc. Cambridge Philos. Soc. 96 (1984) 85-93. MR0743704 (85j:55018)

[12] P. Goerss, H.-W. Henn, M. Mahowald, The homotopy of $L_{2} V(1)$ for the prime 3, Categorical decomposition techniques in algebraic topology (Isle of Syke, 2001), Progr. Math. 215 (2004) 125-151, Birkhäuser, Basel.

[13] P. Goerss, H.-W. Henn, M. Mahowald, C. Rezk, A resolution of the K(2)-local sphere., preprint available from http://www.hopf.math.purdue.edu (2002).

[14] B. Gray, The periodic lambda algebra, Stable and unstable homotopy (Toronto, ON, 1996), Fields Institute Comm. 19 (1998) 93-101. MR1622340 (99c:55014)

[15] J.P.C. Greenlees, J.P. May, Generalized Tate cohomology, Mem. Amer. Math. Soc., Vol. 113, 1995. MR1230773 (96e:55006)

[16] J.P.C. Greenlees, H. Sadofsky, The Tate spectrum of $v_{n}$-periodic complex oriented theories, Math. Z. 222 (1996) 391-405. MR.1400199 (97d:55010)

[17] J.H. Gunawardena, Segal's conjecture for cyclic groups of odd prime order, J.T. Knight Prize Essay, Cambridge, 1980.

[18] I. Johnson, Factoring $2^{k}$ on stunted projective spectra and the root invariant, Topology Appl. 141 (2004) 21-57. MR2058680(2005a:55014)

[19] A. Lawrence, Higher order compositions in the Adams spectral sequence, Bull. Amer. Math. Soc. 76 (1970) 874-877. MR0258041 (41:2688)

[20] W.H. Lin, On conjectures of Mahowald, Segal, and Sullivan, Math. Proc. Camb. Phil. Soc. 87 (1980) 449-458. MR0556925 (81e:55020)

[21] W.H. Lin, D.M. Davis, M. Mahowald, J.F. Adams, Calculation of Lin's Ext groups. Math. Proc. Cambridge Philos. Soc. 87 (1980) 459-469. MR0569195 (81e:55025)

[22] M. Mahowald, The metastable homotopy of $S^{n}$, Memoir Amer. Math. Soc., Vol. 72, 1967. MR0236923 (38:5216)

[23] M. Mahowald, The image of $J$ in the EHP sequence, Ann. of Math. 116 (1982) 65-112. MR.0662118 (83i:55019)

[24] M. Mahowald, D. Ravenel, Toward a global understanding of the homotopy groups of spheres, Proceedings of the Lefschetz Conference, Mexico (1984) 57-74. MR0893848 (88k:55011)

[25] M. Mahowald, D. Ravenel, The root invariant in homotopy theory, Topology 32 (1993) 865898. MR1241877 (94h:55025)

[26] M. Mahowald, P. Shick, Periodic phenomena in the classical Adams spectral sequence, Trans. Amer. Math. Soc. 300 (1987) 191-206. MR0871672 (88e:55019)

[27] M. Mahowald, P. Shick, Root invariants and periodicity in stable homotopy theory, Bull. London Math. Soc. 20 (1988) 262-266. MR0931189 (89b:55009)

[28] H. Miller, On relations between Adams spectral sequences, with an application to the stable homotopy of a Moore space, J. Pure and App. Alg. 20 (1981) 287-312. MR0604321 (82f:55029)

[29] H. Miller, On Jones's Kahn-Priddy theorem, Homotopy theory and related topics, Lecture Notes in Math. 1418, Springer, Berlin, 1990. MR.1048188 (91a:55017)

[30] R.M.F. Moss, Secondary compositions and the Adams spectral sequence, Math. Z. 115 (1970) 283-310. MR.0266216 (42:1123)

[31] S. Oka, Note on the $\beta$-family in stable homotopy of spheres at the prime 3, Kyushu Mem. Fac. Sci. Ser. A 35 (1981) 367-373. MR0628729 (83c:55019)

[32] D. Ravenel, The Segal conjecture for cyclic groups and its consequences, with an appendix by Haynes R. Miller, Amer. J. Math. 106 (1984) 415-446. MR0737779 (85g:55015)

[33] D. Ravenel, Complex Cobordism and the Stable Homotopy Groups of Spheres, Pure and Applied Math., Academic Press, 1986. MR0860042 (87j:55003)

[34] K. Shimomura, X. Wang, The homotopy groups $\pi_{*}\left(L_{2} S^{0}\right)$ at the prime 3, Topology 41 (2002) 1183-1198. MR1923218 (2003g:55020)

[35] H. Sadofsky, The root invariant and $v_{1}$-periodic families, Topology 31 (1992) 65-111. MR.1153239 (92k:55021)

[36] P. Shick, On root invariants of periodic classes in $\operatorname{Ext}_{A}(\mathbb{Z} / 2, \mathbb{Z} / 2)$, Trans. Amer. Math. Soc. 301 (1987) 227-237. MR0879570 (88f:55027) 
[37] B. Shipley, An algebraic model for rational $S^{1}$ equivariant stable homotopy theory, Q. J. Math. 53 (2002) 87-110. MR1887672 (2003a:55026)

[38] R. Thompson, The $v_{1}$-periodic homotopy groups of an unstable sphere at odd primes, Trans. Amer. Math. Soc. 319 (1990) 535-559. MR1010890 (90j:55021)

Department of Mathematics, Massachusetts Institute of Technology, Cambridge, MASSACHuSETtS 02139 